\title{
MATHEMATICAL MODEL OF THE SAVANNAH RIVER SiTE WASTE TANK FARM (U)
}

by

\section{F. G. Smith, III}

Westinghouse Savannah River Company

Savannah River Site

Aiken, South Carolina 29808

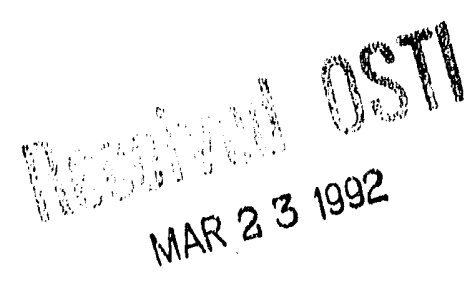

A report to be sent to OSTI and proposed for distribution to the General Public

This report was prepared in connection with work done under Contract No. DE-AC09-89SR18035 with the U.S. Department of Energy. By acceptance of this report, the publisher and/or recipient acknowledges the U.S. Government's right to retain a nonexclusive, royalty-free license in and to any copyright covering this report, along with the right to reproduce and to authorize others to reproduce all or part of the copyrighted report. 
WSRC-RD-91-17

\section{A MATHEMATICAL MODEL OF THE SAVANNAH RIVER SITE WASTE TANK FARM (U)}

Frank G. Smith, III

Westinghouse Savannah River Company

Savannah River Laboratory

Aiken, SC 29808

\section{Prepared for the U.S. Department of Energy under Contract}

DE-AC09-88SR18035 


\section{DISCLAIMER}

This report was prepared by Westinghouse Savannah River Company (WSRC) for the United States Department of Energy under Contract No. DE-AC09-88SR 18035 and is an account of work performed under that contract. Neither the United States Department of Energy, nor WSRC, nor any of their employees makes any warranty, expressed or implied, or assumes any legal liability or responsibility for accuracy, completeness, or usefulness, of any information, apparatus, or product or process disclosed herein or represents that its use will not infringe privately owned rights. Reference herein to any specific commercial product, process, or service by trademark, name, manufacturer or otherwise does not necessarily constitute or imply endorsement, recommendation, or favoring of same by WSRC or by the United States Government or any agency thereof. The views and opinions of the authors expressed herein do not necessarily state or reflect those of the United States Government or any agency thereof.

Printed in the United States of America

Available from

National Technical Information Service

U. S. Department of Commerce 5285 Port Royal Road

Springfield, VA 22161 


\section{WESTINGHOUSE INTERNAL DISTRIBUTION}

G. T. Wright, $703-\mathrm{H}$

M. A. Ceravolo, $703-\mathrm{H}$

P. D. d'Entremont, 703-H

G. K. Georgeton, 703-H

B. L. Lewis, 703-H

T. E. Pate, $703-\mathrm{H}$

M. S. Peters, 703-H

WMT File Code 179.0, 703-H

M. J. Barnes, 773-A

I. G. Choi, 773-59A

D. T. Hobbs, 773-A

D. D. Walker, 773-A

F. G. Smith (2), 773-11A

SRL Records (4), 773-A 
WSRC-RD-91-17

KEYWORDS: TANK FARMS

WASTE TANKS

MODELS

COMPUTER CODES

RETENTION: PERMANENT

\section{A MATHEMATICAL MODEL OF THE SAVANNAH RIVER SITE WASTE TANK FARM (U)}

F. G. Smith, III

Publication Date: July 15, 1991

Westinghouse Savannah River Company

Savannah River Laboratory

Aiken, SC 29808 


\section{CONTENTS}

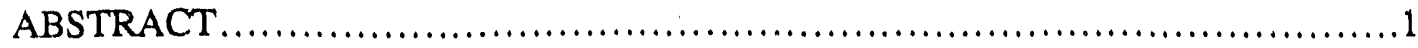

SUMMARY .............................................................



EVAPORATOR SYSTEM MATHEMATICAL MODELING ..........................11

Evaporator Material Balance..............................................12

Evaporator Receiving Tank Material Balance.....................................14

Evaporator Feed Tank Material Balance...........................................16

Solution of the Material Balance Equations ...................................18

Treatment of 1F-2F Dual Evaporator Systern ..........................21

Special Case of No Input iu Evaporator Feed Tank ...................23

Special Case When the Evaporator is not Functioning ...................24

Treatment of Variable Receiving Tank Specific Gravity .................24

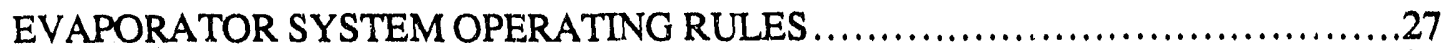

Concentrate Receiving Tanks..............................................27

Evaporator Operation ........................................................28

Low Heat Waste Sources ................................................28

High Heat Waste Receipt Tanks .........................................29

Concentrate Recycle ......................................................... 31

Waste Transfers........................................................ 31

DETERMINATION OF CANYON WASTE GENERATION....................... 32

WASTE TANKS OUTSIDE OF EVAPORATOR SYSTEMS ............................33

MODEL COMPUTER PROGRAMS .............................................34

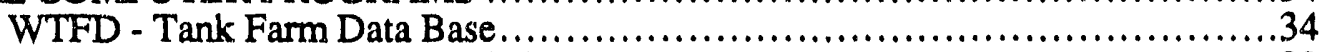

WATFAM - Simulation Calculation ............................................. 38

READER - Simulation Output Retrieval ..................................42

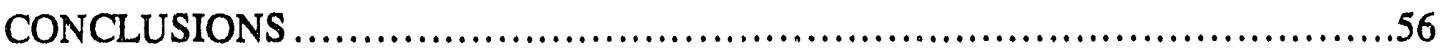

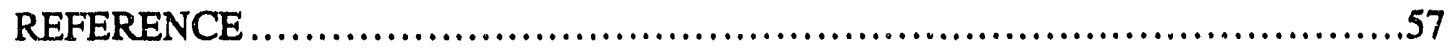

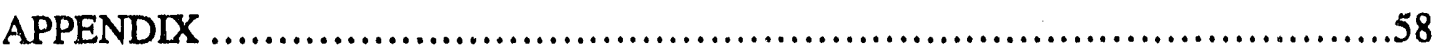

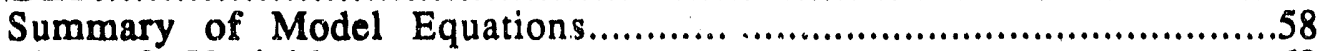

List of Variables............................................................6 60

User's Guide to Model Computer Frograms .................................61

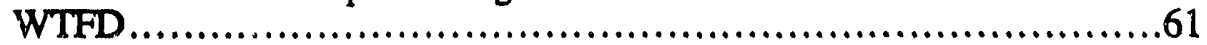

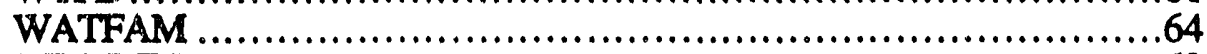

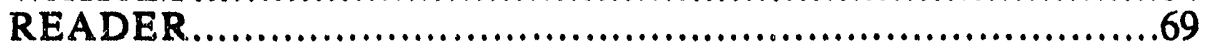

Partial Listing of Tank Farm Data Base $\ldots \ldots \ldots \ldots \ldots \ldots \ldots \ldots \ldots \ldots \ldots \ldots \ldots \ldots \ldots . .72$ 


\section{List of Figures}

Figure 1a. Schematic representation of SRS F-Area waste tank farm.

Figure 1b. Schematic representation of SRS H-Area waste tank farm as coded in the model.

Figure 1c. Schematic representation of SRS H-Area waste tank farm as currently operating.

Figure 2. Evaporator system model flow schematic.

Figure 3. Evaporator flow schematic.

Figure 4. Evaporator receiving tank flow schematic.

Figure 5. Evaporator feed tank flow schematic.

Figure 6. Schematic representation of mixing or separating a salt solution.

Figure 7. Screen display during a sample run of program WATFAM.

Figure 8. Partial listing of full log file for the 2F evaporator system (2F.LOG) from a four year simulation.

Figure 9. Full listing of short log files for all four evaporator systems created from a four year simulation.

Figure 10a. Screen display of the menu from program READER.

Figure 10b. Screen display with Option 1 of program READER.

Figure 11. Listing of model output for tank 43 from a four year simulation.

Figure 12. Model predictions of waste volumes in tank 38.

Figure 13. Model simulation output for Type III waste tanks.

Figure 14. Model predictions of waste volume within all waste tanks over a four year interval.

Figure 15. Model simulation output for the $2 \mathrm{H}$ evaporator.

Figure 16. Model simulation of $2 \mathrm{H}$ evaporator operation over a four year period.

Figure 17. Model simulation of $2 \mathrm{H}$ evaporator net space gain and utility over a four year period. 


\section{List of Figures (continued)}

Figure 18. Complete listing of yearly summary data for the $2 \mathrm{H}$ evaporator system from a four year simulation.

Figure 19. Four year model simulation of waste volumes in the $2 \mathrm{H}$ evaporator system.

Figure 20. Four year model simulation of waste capacity in the $2 \mathrm{H}$ evaporator system.

Figure 21. Material inflow-outflow summary for model predictions of total waste and salt cake volumes processed by the tank farm evaporator systems in fiscal year 1987.

Figure A1. Diagram showing key functions active for WTFD editing.

Figure A2a. Partial structure chart for WATFAM code.

Figure A2b. Continuation of structure chart for WATFAM code.

Figure A3. Structure chart for READER code.

Figure A4. Screen display of process dates.

Figure A5. Screen display of first page of input data specifying waste tank initial conditions.

Figure A6. Screen display of evaporator system input parameters.

Figure A7. Screen display of input data to order the usage of the evaporator receiving tanks.

Figure A8. Screen display showing input parameters for canyon waste streams.

Figure A9. Screen displays showing the input data for the MMP forecast and evaporator fractional utilities on a monthly and yearly basis.

Figure A10. Screen display for input of salt removal schedule for tank 25.

Figure A11. Screen display for input of sludge removal schedules for tanks 21 and 22.

Figure A12. Screen displays showing input data for miscellaneous waste volume additions to each evaporator system on a monthly and yearly basis.

Figure A13. Screen display for miscellaneous input parameters. 


\title{
A MATHEMATICAL MODEL OF THE SAVANNAH RIVER SITE WASTE TANK FARM
}

\begin{abstract}
A mathematical model has been developed to simulate operation of the waste tank farm and the associated evaporator systems at the Savannah River Site. The model solves material balance equations to predict the volumes of liquid waste, salt, and sludge for all of the tanks within each of the evaporator systems. Additional logic is included to model the behavior of waste tanks not directly associated with the evaporators. Input parameters include the Material Management Plan forecast of canyon operations, specification of other waste sources for the evaporator systems, evaporator operating characteristics, and salt and sludge removal schedules. The model determines how the evaporators will operate, when waste transfers can be made, and waste accumulation rates. Output from the model includes waste tank contents, summaries of system operations, and reports of space gain and the remaining capacity to store waste materials within the tank farm. Model simulations can be made to predict waste tank capacities on a daily basis for up to 20 years. .The model is coded as a set of three computer programs designed to run on either IBM ${ }^{\star}$ compatible or Apple ${ }^{\otimes}$ Macintosh $^{\otimes}$ II personal computers.
\end{abstract}




\section{SUMMARY}

Current forecasts indicate that the waste tank farm at the Savannah River Site will be utilized at close to full capacity in the near future. To accurately estimate the tank farm's capacity to handle projected waste volumes, a computer model was developed to simulate the operation of the evaporator systems and other processing operations associated with the waste tank farm. The model solves a set of transient material balance equations to predict the volumes of liquid waste, salt cake, and sludge present in the waste tanks within the evaporator systems. Simple models of in-tank precipitation and sludge processing were also included to provide a complete analysis for all waste tanks within the tank farm. The model estimates evaporator operational characteristics, waste transfer scheduling, and waste accumulation rates. Model simulations can be made to predict waste tank capacities on a daily basis for up to 20 years. Use of a one day time increment for the model calculations is sufficiently accurate for the long term predictions of tank farm operating characteristics that are of most interest.

The Savannah River Site tank farm uses four evaporator systems to reduce the volume of radioactive wastes. Two low heat waste evaporators (1F and $2 \mathrm{~F})$ are located in F-Area and one high heat waste evaporator $(1 \mathrm{H})$ and one low heat waste evaporator $(2 \mathrm{H})$ are located in $\mathrm{H}$-Area. Twenty one waste storage tanks are directly associated with the evaporator operations. Waste material from the canyons and other sources is sent to the feed tanks for each evaporator. The evaporators reduce the waste volume by removing water and send concentrated waste to one of several receiving tanks. The concentrate receiving tanks are cooled to precipitate out salt cake from the high specific gravity liquid. Supernate is recycled from the receiving tanks back to the evaporator feed tank for additional volume reduction. When one million gallons of salt cake accumulate in a receiving tank, the tank can no longer be used to receive concentrated waste until the salt has been removed.

Modeling of the evaporator systems is accomplished by solving time dependent mass conservation equations for total waste, salt, and sludge around the evaporators, concentrate receiving tanks, and feed tanks. The volume of liquid waste in the tanks, the liquid specific gravity, and salt mass fraction are all functions of time as waste material is mixed into and removed from the tanks. A semi-analytical solution to the set of material balance equations was obtained under very general conditions and is combined with an extensive set of rules that describe operational considerations in the evaporator systems to complete the model. The operating rules specify when waste transfers occur, when concentrate recycle takes place, and when concentrate receiving tanks are switched. Operating rules are also required to control the flow of fresh high heat waste into the $1 \mathrm{H}$ evaporator system, to determine when the evaporators are in operation, and to decide when to make inter-area line transfers.

The principal source of waste for the evaporator systems is canyon operations in $F$ and $H$ areas. Canyon waste generation is calculaced as a fixed monthly amount plus an amount that depends on material processing. The Materials Management Plan estimates of the metric tons of uranium and number of reactor tubes to be processed in the separations areas are input to the model and used to calculate canyon waste production. The model separates sludge from the canyon waste streams and deposits it in the evaporator feed tanks. Sources of additional waste volume such as descale and flush vvarer in the evaporators and steam jet dilution during waste transfers are also accounted for:

In addition to the evaporator system operations, some of the waste tanks in the tank farm are used for other processing functions and simple models of these operations have been included in the simulation. In particular, the RBOF (tanks 21 and 23), sludge washing 

(tanks 40,42 , and 51 ), and in-tank precipitation (tanks 48,49 , and 50) processes are
modeled.

The computer model is programmed in a set of three individual codes:

- WTFD a BASIC program used to create and modify the tank farm data base that provides input for the model simulations. Since the data base is fairly large, it is convenient to separate the creation and writing of the data from the model calculations. The WTFD program is designed to facilitate entry and modification of model input data. Input data is contained in a file named TFARM.DAT. The input data consists of:

1) Dates for the following processes:

1. Simulation start.

2. Beginning of waste removal from type IV tanks.

3. Ending of waste removal fiom type IV tanks.

4. F-Area canyon evaporator change over.

5. 1H evaporator upgrade.

6. Sludge wash water startup.

7. DWPF startup.

2) Tank level in inches of salt, sludge, and total volume, and the specific gravity of the tank supernatant liquid for the 51 waste tanks in the tank farm on the starting date.

3) Operating parameters for the four existing evaporators and new $1 \mathrm{H}$ evaporator consisting of: overhead production, lance steam addition, a basis specific gravity, flush water ratio, recycle specific gravity, and the feed tank number.

4) The order in which concentrate receiving tanks are to be used in each evaporator system.

5) Parameters for low and high heat waste streams from $F$ and $\mathrm{H}$ area canyons cunsisting of: the fixed volume of waste generated each month independent of production, the volume of waste directly a function of canyon production, the salt and sludge content of the waste streams.

6) Canyon production (reactor tubes processed in H-Area and metric tons of uranium processed in F-Area) and evaporator utilities.

7) A schedule for the removal of salt cake from old waste tanks and from the concentrate receiving tanks giving removal dates and the volume of salt cake removed.

8) A schedule for the removal of sludge from the waste tanks giving removal dates and the volume of sludge removed.

9) Waste volume and salt additions to each evaporator system from sources other than those directly included in the process model. 
10) Miscellaneous operating parameters consisting of: volumes and specific gravities of waste generated by the retirement of old waste tanks, flow rates, specific gravities, and sludge content of the DWPF recycle and sludge wash water waste streams, specification of the active fresh waste receipt tank in the high heat waste systems and the age of the waste held in other high heat receiver tanks.

- WATFAM a FORTRAN program that encodes the mathematical model and performs the actual calculation of the tank farm simulation. The program user specifies the total number of days to run the simulation, the desired printout interval, a random number seed used to determine evaporator down time, and the type of output log files desired. Output from the execution of WATFAM is written to data files TANK.DAT and EVAP.DAT in binary format. ASCII $\log$ files indicating the status of various process functions are also written for each evaporator system to record: dates when waste transfers, concentrate recycling, and inter-area line transfers occur, dates when concentrate receiving tanks fill, the status of evaporator operation, and error messages.

- READER a FORTRAN program designed to read the TANK.DAT and EVAP.DAT output files created by WATFAM and to organize the data into convenient tabular formats that convey useful summaries of the calculated results to the user. Copies of the summary tables can be saved in ASCII output files. The READER program provides five options for displaying the simulation output data:

1) Volumes of total waste, salt cake, and sludge in kgal and in inches of tank level and the specific gravity of the liquid waste for individual tanks.

2) Volume data for all waste tanks of a particular type (I, II, III, or IV), for the four fresh waste receiving tanks in the high heat system, or for all waste tanks in the tank farm. The volumes of total waste, salt cake, and sludge are given in $\mathrm{kgal}$ and in inches of tank level.

3) Average flow rates in $\mathrm{kgal} /$ day of overhead, concentrate, and feed streams, feed specific gravity, net space gain in kgal, and percent operation for a particular evaporator.

4) Volumes of total waste, salt cake, and sludge, available storage volume for total waste and salt cake, and net space gain in $\mathrm{kgal}$ for particular evaporator systems.

5) A summary report showing the volume of waste and salt cake flowing into and out of each evaporator system with the associated waste accumulation and space gain on a monthly or yearly basis.

For summary purposes, three evaporator systems $(1 \mathrm{~F} / 2 \mathrm{~F}, 1 \mathrm{H}$, and $2 \mathrm{H})$ are reported. The data for the $1 \mathrm{~F} / 2 \mathrm{~F}$ system includes tanks $26,44,45,47,25$, 27 , and 28. Data for the $1 \mathrm{H}$ system uses tanks $30,13,32$, and receiving 
tanks $29,31,36$, and 37 . The $2 \mathrm{H}$ system data includes tanks 43,41 , and 38.

Some of the assumptions and limitations present in the current model are:

- A simple empirical model based on past operating experience was used to estimate evaporator performance rather than a physical model of the evaporator operation.

- Steady state evaporation producing concentrate at a constant specific gravity is assumed to occur. The model treats the waste mixture as an isothermal ideal solution and assumes that liquid and salt do not accumulate within the evaporator.

- The liquid specific gravity and mass fraction of salt in the salt cake within the evaporator receiving tanks are assumed to be constant. The model also assumes that equilibrium is immediately established between the salt cake and supernatant liquid in the receiving tanks.

- No temperature dependence is included in the model.

- The model flow sheet was based on 1988 operations and should be updated to reflect changes in tank farm processes.

The model is designed to run on a personal computer and versions compatible with IBM ${ }^{\star}$ and Apple ${ }^{\otimes}$ Macintosh II computers are available. This document describes the derivation of the mathematical model, gives a complete description of the computer codes developed to implement the model with instructions for their use, and shows some typical example calculations. 


\section{A MATHEMATICAL MODEL OF THE SAVANNAH RIVER SITE WASTE TANK FARM}

\section{INTRODUCTION}

Current forecasts indicate that the waste tank farm at the Savannah River Site will be utilized at close to full capacity in the near future. To accurately assess the available tank farm capacity, a dynamical computer model capable of simulating the operation of the waste tank farm evaporator systems and of predicting waste volumes in all tanks within the tank farm was developed. The model predicts the salt, sludge, and liquid waste volumes within the waste tanks on a daily basis. Model projections of waste tank capacity may be calculated for up to 20 years.

The Savannah River Site tank farm uses four evaporator systems to reduce the volume of radioactive wastes. Twenty one waste storage tanks are directly associated with evaporator operations. A schematic diagram of the waste tank farm material flow as it is coded in the model is shown in Figs. $1 \mathrm{a}$ and $1 \mathrm{~b}$. (Since the $\mathrm{m}$-del was written, operations in the HArea high heat waste processing have changed slightly and a revised flow sneet showing the current configuration is given in Fig. 1c. Differences between the model simulation and current operating practice will be pointed out as they occur in the following discussion.) Two waste evaporators (designated as $1 \mathrm{~F}$ and $2 \mathrm{~F}$ ) are located in F-Area and two evaporators $(1 \mathrm{H}$ and $2 \mathrm{H})$ are located in $\mathrm{H}$-Area. Waste material from various sources is sent to the feed tanks for each evaporator. The evaporators reduce the waste volume by removing water as overheads and send cencentrated waste to one of several receiving tanks. The concentrate receiving tanks are cooled to precipitate out salt cake from the high specific gravity waste. Supernate is reciycled from the receiving tanks back to the evaporator feed tank for further volume reduction. After one million gallons of salt cake have accumulated in a concentrate receiving tank, the tank is no longer used to receive concentrated waste until the salt cake has been removed. Evaporator overheads are further processed in the Effluent Treatment Facility (ETF). Overheads from the evaporation of high heat waste are passed through the Cesium Removal Column (CRC) before going to the ETF.

As Fig. 1a indicates, low heat waste (LHW) from the F-Area canyon is sent to tank 26 which is used to feed both the $1 \mathrm{~F}$ and $2 \mathrm{~F}$ evaporators. Other sources of waste input to tank 26 are the removal of excess liquid from the old type IV F-Area waste tanks and the transfer of LHW supernate from H-Area through the inter-area line. The $2 F$ evaporator sends concentrate to receiving tanks 44,45 , and 47 while the $1 \mathrm{~F}$ evaporator uses tanks 25 , 27, and 28. Supernate from all of these concentrate tanks is recycled back to tank 26 for further volume reduction.

Low heat waste from $\mathrm{H}$-Area canyon operations is sent to tank 43 which feeds the $2 \mathrm{H}$ evaporator as shown in Fig. 1b. Excess liquid removed from old type IV waste tanks in $\mathrm{H}$-Area is also sent to tank 43 . In adjition, wash water from sludge processing and recycle from the Defense Waste Processing Facility (DWPF) operations will be sent to tank 43. Since the combination of these additional waste streams represents a significant volume, a provision is made to be able to transfer waste out of tank 43 to tank 26 using the inter-area line. The $2 \mathrm{H}$ evaporator sends concentrate to receiving tanks 38 or 41 and supernate from these tanks is recycled back to tank 43. 


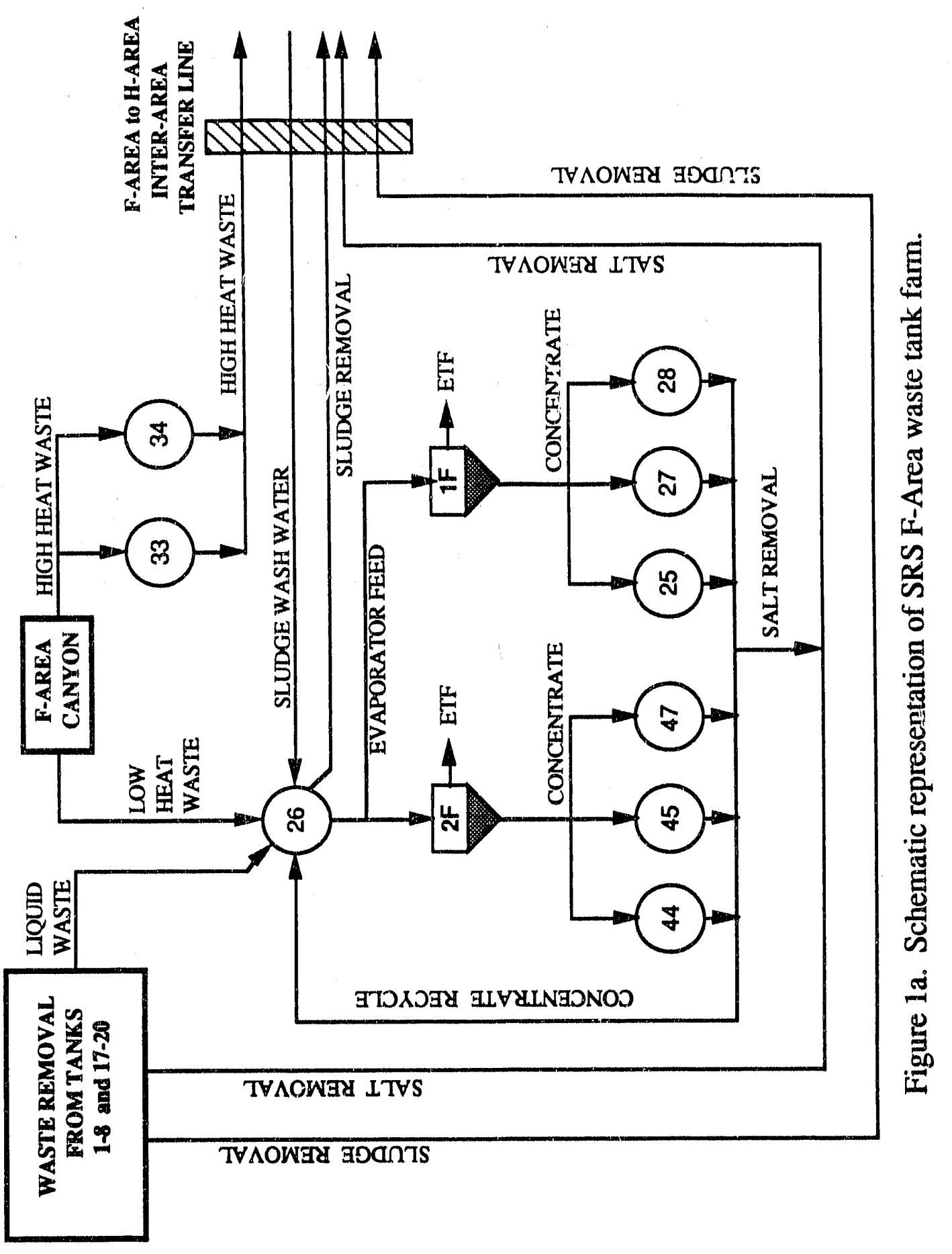




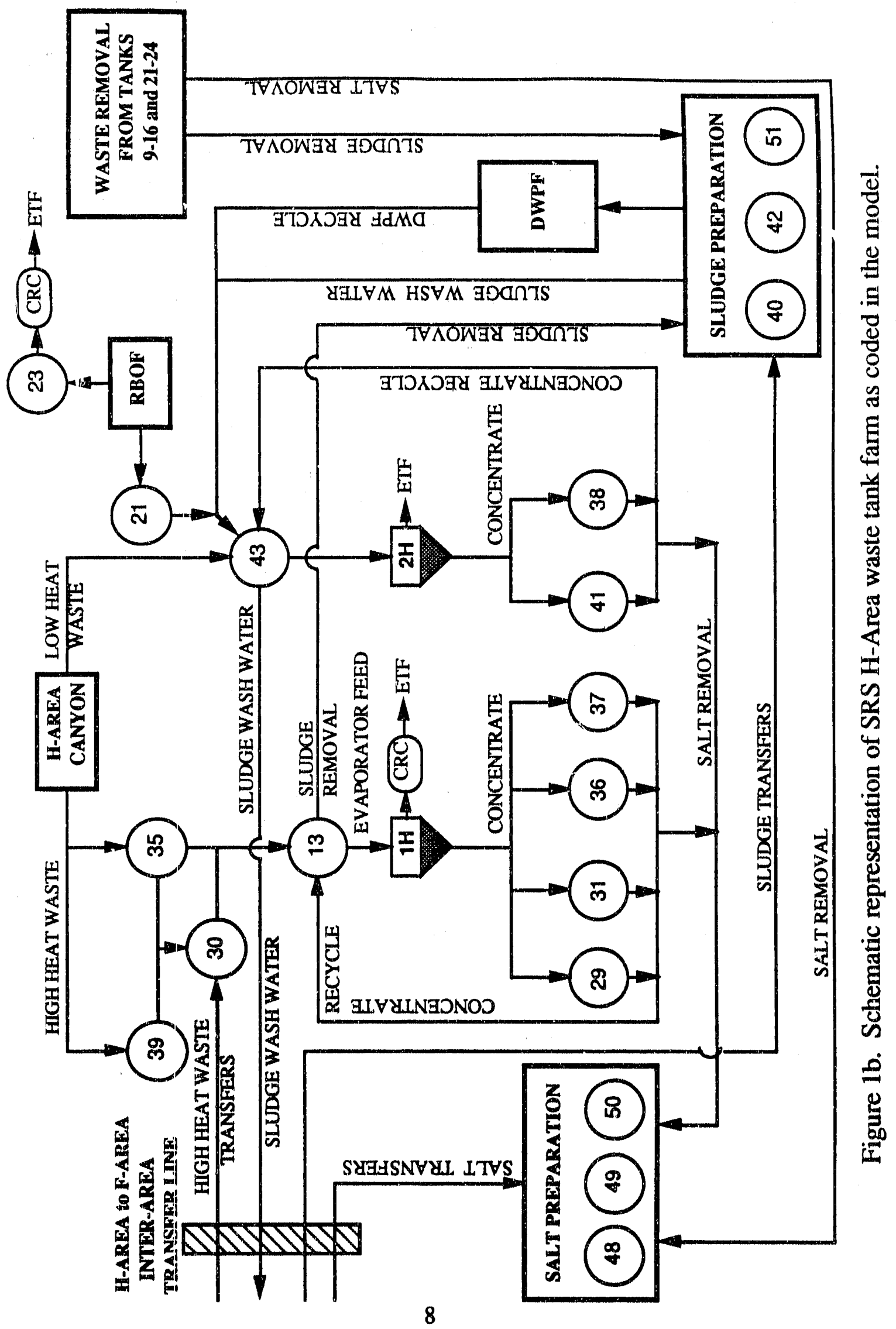




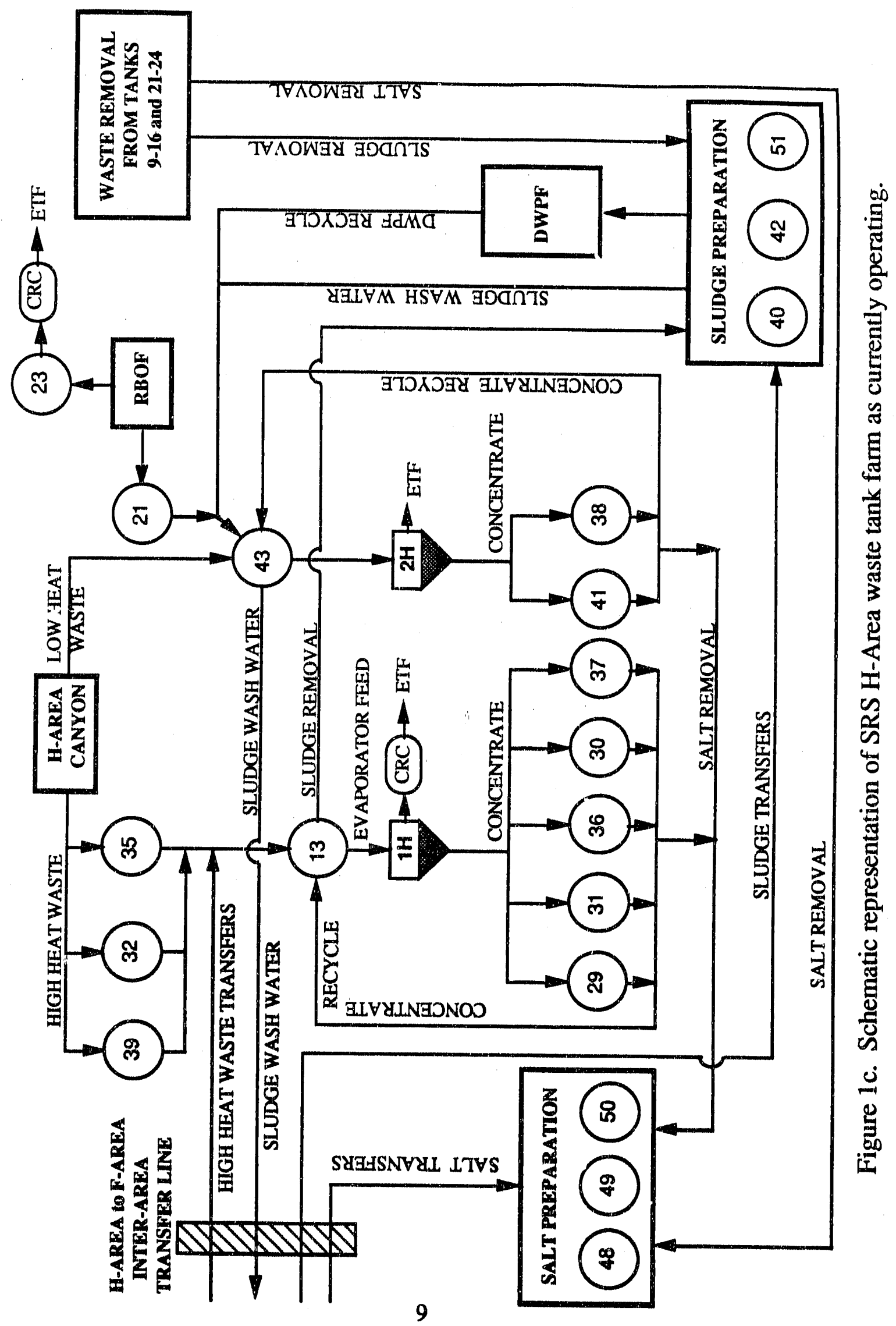


High heat waste (HHW) from both F-Area and $\mathrm{H}$-Area canyon operations is processed in the $1 \mathrm{H}$ evaporator system. The high heat waste must be aged for one year to reduce its radioactivity to acceptable leve:ls before evaporation. Therefore, this material is initially accumulated in a set of fresh waste receipt tanks before being sent to the $1 \mathrm{H}$ evaporator feed tank. Tanks 33 and 34 are used to accumulate fresh high heat waste in F-Area and tanks 35 and 39 are used in H-Area. As shown in Fig. 1c, tank 32 is now a high heat waste receiver in $\mathrm{H}$-Area although it was not included in this capacity in the current model. High heat waste from F-Area is transferred through the inter-area line to tank 30 after it has aged for at least one year. The model also transfers the contents of tank 39 into tank 30 before feeding it to the evaporator. Liquid waste in tanks 35 and 30 is transferred into tank 13 to feed the $1 \mathrm{H}$ evaporator and the simulation transfers liquid from tank 35 into tank 30 to maximize use of the available storage space. Basically, the model transfers waste from any of the HHW receivers into either tank 13 or 30 to get it into the $1 \mathrm{H}$ system. Actual current operations (shown in Fig. 1c) only transfer waste from the receiving tanks into tank 13 and use tank 30 as an evaporator concentrate receiving tank.

The model assumes that the $1 \mathrm{H}$ evaporator uses tanks $29,31,36$, and 37 as concentrate receiving tanks. When these tanks have all accurnulated one million gallons of salt cake, tank 30 will be switched and used to receive concentrated waste. At this time, the model will replace tank 30 with tank 32 (not shown in Fig. 1b) as a fresh high heat waste receiving tank. It is expected that the $1 \mathrm{H}$ evaporator will be replaced with a larger capacity unit around 1994. When this occurs, tank 32 will replace both tanks 30 and 13 in the flow schematic and most of the excess waste from the $2 \mathrm{H}$ evaporator will be sent to the new $1 \mathrm{H}$ evaporator rather than to F-Area. While these modifications are not shown on the flow schematic, logic for their implementation is included in the computer model.

Changes in tank farm operations since the model was written make some of the modeling assumptions explained in the preceding paragraph invalid. As shown in Fig. 1c, tank 30 is being used as a concentrate receiving tank for the $1 \mathrm{H}$ evaporator and tank 32 is used as a fresh high heat waste receiver. Current plans would continue this operation and would not replace tank 13 as the $1 \mathrm{H}$ evaporator feed tank. DWPF recycle may also be sent straight to tank 32 instead of going through 43 first as shown on the flow sheets. Some modifications to the model code would be required to change the simulation to reflect these new operating characteristics.

In addition to the evaporator system operations, some of the waste tanks are involved in other processing functions. Tanks 21 and 23 are used in conjunction with the Receiving Basin for Offsite Fuels (RBOF) processing which generates some low activity waste that is fed to the $2 \mathrm{H}$ evaporator. The waste in tank 23 can also be combined with $1 \mathrm{H}$ overheads and be processed through the Cesium Removal Column before going to the Effluent Treatment Facility. When sludge is removed from the waste tanks, it will be washed with water to reduce the concentration of soluble salts before it is fed to the DWPF. Tanks 40 , 42 , and 51 will be used for the sludge processing which generates a significant volume of low activity wash water. Salt cake that is removed from the waste tanks will be processed in tanks 48, 49, and 50 using in-tank precipitation. Precipitation and washing are to be performed in tanks 48 and 50 while tank 49 will be used to hold precipitate prior to transfer to DWPF for vitrification along with washed sludge. Simple models of these processing operations have been included in the model.

A detailed description of the mathematical modeling used to simulate operation of the tank farm evaporator systems is presented in the following section. Derivations of the basic material balance equations are given explaining the assumptions and simplifications involved and their implementation in the computer programs. The simulation must also follow certain operating rules that physically constrain the waste tank farm processes. 
These rules are discussed in the section of the report following the equation derivations. The next sections explain the calculations used to determine canyon waste generation and the simple modeling used for tanks that are not associated directly with the evaporator systems. Following this, a section is included that provides a detailed description of the three computer programs that implement the modeling. In the discussion of the program READER that is used to retrieve model output, typical model results are presented in both tabular and graphical displays. Finally, a brief conclusion is presented summarizing the status of the modeling effort. A summary of the model equations and some of the input data needed to run a simulation have been included in an Appendix to the report.

\section{EVAPORATOR SYSTEM MATHEMATICAL MODELING}

Mathematical modeling of the evaporator systems is based on solving the material balance equations for conservation of the total waste mass and salt mass around the evaporator, concentrate receiving tank, and feed tank. A schematic representation of a complete evaporator system is shown in Fig. 2. The material balance equations are written assuming steady state evaporator operation. An analytical solution to the set of material balance equations has been obtained under very general conditions. This solution of the material balance equations is combined with rules for operating constraints on the waste tank farm to completely model the overall tank farm evaporator system.

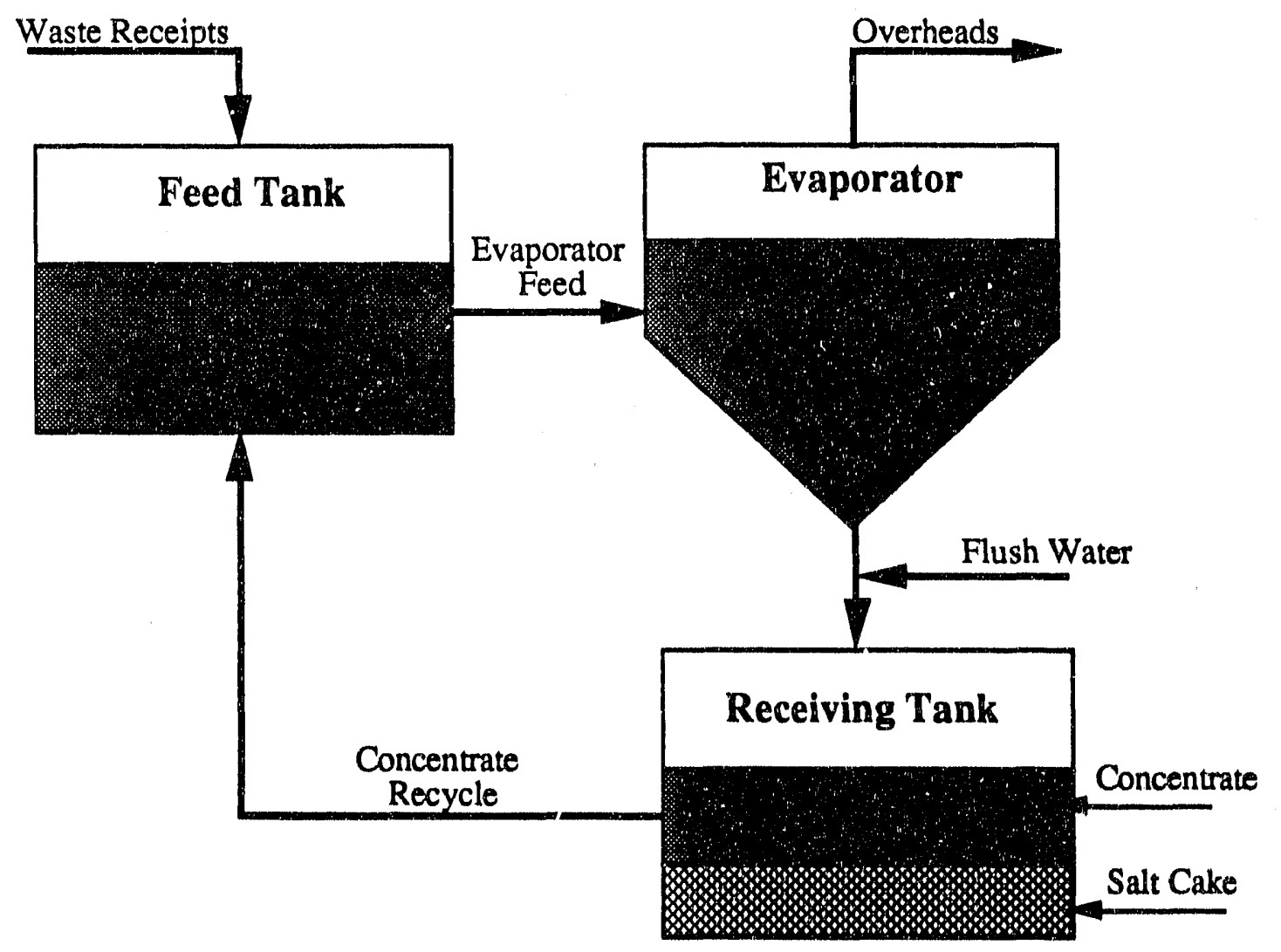

Figure 2. Evaporator system model flow schematic. 
A basic assumption in the material balance calculations is that the specific gravity of liquid waste material (SPG) can be related to the mass fraction of salt in the waste $\left(\mathrm{X}_{\mathrm{salt}}\right)$ as

$$
\mathrm{SPG}=\frac{1}{\left(1-\mathrm{K}_{\mathrm{a}} \mathrm{X}_{\mathrm{salt}}\right)}
$$

where $\mathrm{K}_{\mathrm{a}}$ is a constant equal to 0.5455 for Savannah River Site waste material. Equation (1) was derived from the following three assumptions:

1. The specific gravity of water $\left(X_{\text {salt }}=0\right)$ is 1.0 .

2. The specific gravity of bone dry salt $\left(X_{\text {salt }}=1\right)$ is 2.2 (this determines $K_{a}$ ).

3 . There is no volume change when water and salt are mixed.

Salt cake that accumulates in the receiving tanks is further assumed to contain 13.5 pounds of dry salt in one gallon. Assuming no volume change in the mixture then fixes the water content in one gallon of salt cake at 2.19 pounds (using $\mathrm{PH}_{2} \mathrm{O}=8.324 \mathrm{lbm} / \mathrm{gal}$ ) which gives $\mathrm{X}_{\text {salt }} \equiv 0.86$. The specific gravity of salt cake is then determined to be 1.885 from Eq. (1).

Sludge that is present in waste streams is treated separately from the dissolved salt solution. The sludge is assumed to immediately settle out in the fresh waste receipt tanks. Therefore, a material balance on sludge simply involves adding the volume of sludge to the waste receipt tank and subtracting any sludge removal volume. This calculation is performed independently of the other material balances.

\section{Evaporator Material Balance}

A schematic diagram of a waste evaporator and the associated flow streams is shown in Fig. 3. The evaporator receives liquid feed at volumetric flow rate $V_{f}$ and reduces the volume by driving off water at rate $V_{0}$. Concentrated waste leaves the evaporator at volumetric flow rate $V_{c}$. An overall material balance for steady state operation is then given by

$$
V_{f} \rho_{f}=V_{o} \rho_{o}+V_{c} \rho_{c}
$$

where $\rho_{f}, \rho_{o}$, and $\rho_{c}$ are the mass densities of the evaporator feed, overhead (water), and concentrate streams, respectively. Steady state operation assumes that no material accumulates within the evaporator. 


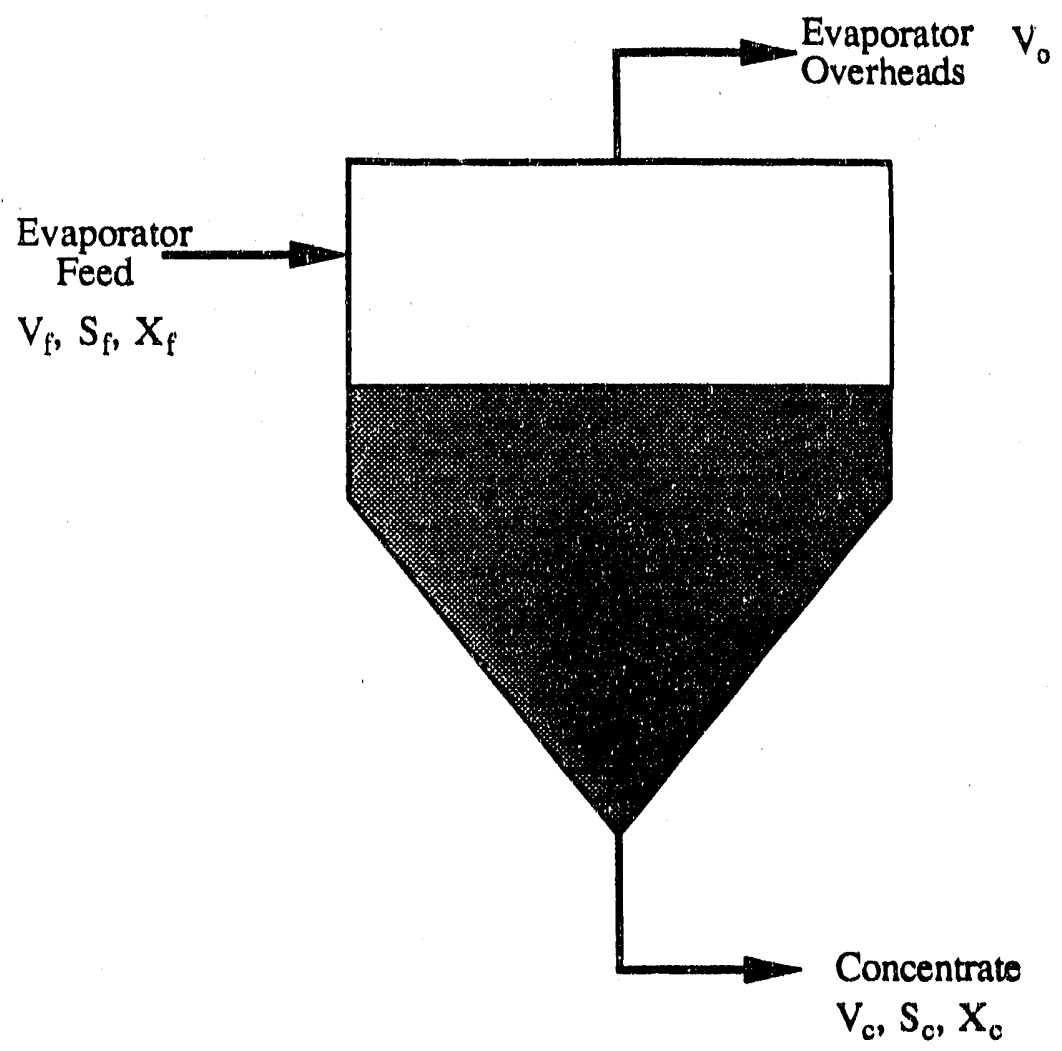

Figure 3. Evaporator flow schematic.

It is convenient to develop the material balance equations in terms of specific gravities. Dividing Eq. (2) by $\rho_{0}$, the density of water, gives

$$
V_{f} S_{f}=V_{o}+V_{c} S_{c}
$$

where $S_{f}$ and $S_{c}$ are the specific gravities of the feed and concentrate streams, respectively.

A material balance for the salt can be written as

$$
V_{f} S_{f} X_{f}=V_{c} S_{c} X_{c}
$$

where $X_{f}$ and $X_{0}$ are the mass fractions of salt in the feed and concentrate streams, respectively. To simplify the analysis, we assume that the waste evaporators are operated such that the concentrate specific gravity is always maintained at the constant value of $S_{c}=$ 1.54 in all four evaporator systems. This assumption is generally valid although low specific gravity feed may produce a lower specific gravity concentrate since the change in specific gravity that the evaporators can accomplish is in practice limited by the equipment and operating techniques.

Equations (3) and (4) can be combined to produce the following useful relations 


$$
V_{f} S_{f}=V_{o}\left[\frac{X_{o}}{X_{c}-X_{f}}\right]
$$

and

$$
V_{c} S_{c}=V_{q}\left[\frac{X_{f}}{X_{c}-X_{f}}\right]
$$

If $X_{f}$ and $X_{c}$ are eliminated from Eq. (4) using the formula in Eq. (1), and the resulting expression is combined with Eq. (3), it can be shown that $V_{f}=V_{0}+V_{0}$. That is, the volume of the feed stream to the evaporator is equal to the volume of evaporator overheads plus the volume of concentrated solution. In effect, since the model is isothermal and density variations with temperature are not considered, waste volume is conserved around the evaporation process. The model is then equivalent to treating the waste mixture as an isothermal ideal solution. The treatment also assumes that liquid and salt do not accumulate within the evaporator.

A detailed physical model of the evaporator operation using a heat balance calculation to predict the overhead rates was not developled. In place of a physical model, a simple empirical model based on operating experience with the evaporators was used. The expression used to calculate the net overhead rate is

$$
V_{\mathrm{o}}=\mathrm{K}_{\mathrm{e}}-7560 \mathrm{~S}_{\mathrm{f}}
$$

where $V_{0}$ is given in gallons/day and $K_{0}$ is a constant factor that is determined for each individual evaporator. Equation (7) predicts the net overhead rate while the observed overhead rate is greater than the net rate by the amount of lance steam added to the evaporator. Lance steam is an internal water addition to the evaporator and the material balance equations require use of the net overhead rate. Numerical values used to determine observed overhead rates are presented below in the discussion of the data base management program WTFD.

\section{Evaporator Receiving Tank_Material Balance}

A schematic representation of an evaporator receiving tank and the associated flow streams is shown in Fig. 4. Concentrated liquid waste from the evaporator is fed into the receiving tank and some additional water is added at flow rate $V_{w}$ from desalt and descale flushing of the evaporator. The flow rate of flush water $V_{w}$ is assumed to be directly proportional to the concentrate flow rate $V_{c}$. The constant of proportionality is determined from previous operating experience and is an input parameter to the model calculations. Different constants can be input separately for each evaporator.

Cooling the receiving tank serves to precipitate out salt cake from the concentrated waste. The model assumes that equilibrium is immediately established between the salt cake and supernatant liquid. Wo further assume that the supernatant liquid in the receiving tank maintains a constant specific gravity at a specified value less than 1.54 (the assumed concentrate specific gravity). This liquid specific gravity is a parameter that is also input separately for each evaporator system. While the specific gravity of the supernatant liquid actually depends on the feed chemistry and salt tank temperature, as a simplification we have made provision in the model for only a single representative value to be input for each evaporator. This value is then applied to all receiving tanks in that evaporator system. 
WSRC-RD-91-17

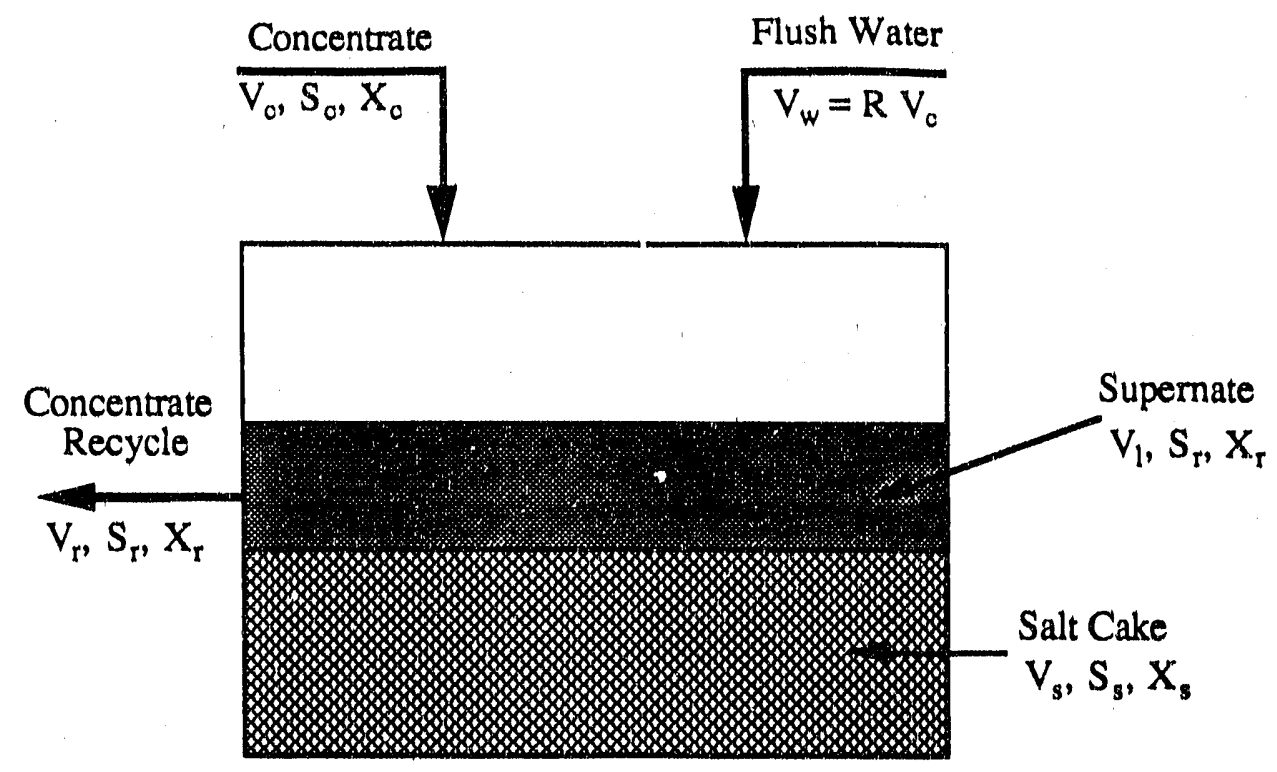

Figure 4. Evaporator receiving tank flow schematic.

If the volume of waste in the receiving tank is approaching the tank operating limit, supernatant liquid is drawn off and recycled to the evaporator feed tank. Recycle from the receiving tank will also take place if the feed tank rieeds liquid volume to keep the evaporator operating. Assuming well mixed conditions, this recycle stream (at volumetric flow rate $V_{r}$, specific gravity $S_{r}$, and salt mass fraction $X_{r}$ ) is of the same composition as the supernatant liquid in the tank. When salt cake is present, the specific gravity of liquid waste in the receiving tank remains constant for each evaporator system.

Since salt and liquid are accumulated in the receiving tank, an unsteady state material balance is appropriate. Instantaneous material balance equations for the total material and the salt are written in terms of specific gravity as

$$
S_{s} \frac{d\left(V_{s}\right)}{d t}+S_{r} \frac{d\left(V_{l}\right)}{d t}=V_{c} S_{c}+R V_{c}-V_{r} S_{r}
$$

and

$$
S_{1} X_{s} \frac{d\left(V_{s}\right)}{d t}+S_{r} X_{r} \frac{d\left(V_{1}\right)}{d t}=V_{c} S_{0} X_{c}-V_{r} S_{r} X_{r}
$$

In Eqs. (8) and (9), $V_{s}$ is the volume of salt cake in the receiving tank, $V_{1}$ is the liquid volume in the tank, $S_{g}$ is the salt cake specific gravity, and $X_{s}$ is the mass fraction of salt in salt cake. In deriving these equations, densities have been converted to specific gravities, the substitution $V_{W}=R V_{c}$ where $R$ is a constant of proportionality has been made, and the assumption that $S_{3}, X_{3}, S_{r}$, and $X_{r}$ have constant values for a particular evaporator has been applied. The specific gravity $S_{r}$ and salt mass fraction $X_{r}$ in the receiving tank liquid phase are in reality not constant values but, as noted above, will vary with feed chemistry and tank temperature. The current model does not track this variation in liquid properties but assumes that these properties remain constant to simplify the solution. 
Equations (8) and (9) can be reorganized into separate equations for the time rate of change of $V_{s}$ and $V_{1}$. Multiplying Eq. (8) by $X_{r}$ and subtracting from Eq. (9), the resulting expression can be rearranged to yield

where

$$
\frac{d\left(V_{s}\right)}{d t}=K_{1} V_{0} S_{o}=K_{1} V_{0}\left[\frac{X_{f}}{X_{c}-X_{f}}\right]
$$

where

$$
K_{1}=\frac{S_{c}\left(X_{c}-X_{r}\right)-R X_{r}}{S_{c} S_{s}\left(X_{s}-X_{r}\right)}
$$

is a constant term and Eq. (6) has been used to write the second equality in Eq. (10).

Substituting Eq. (10) into Eq. (8) and rearranging the result yields

$$
\frac{d\left(V_{1}\right)}{d t}=K_{2} V_{c} s_{c}-V_{r}=K_{2} V_{o}\left[\frac{X_{f}}{X_{c}-X_{F}}\right]-V_{r}
$$

$$
K_{2}=\frac{S_{c}\left(X_{s}-X_{c}\right)+R X_{s}}{S_{c}} \frac{S_{r}\left(X_{s}-X_{r}\right)}{2}
$$

Equations (10) and (11) may then be integrated to obtain values for $V_{s}$ and $V_{1}$ individually if the functional dependence of $X_{f}$ on time is determined. Alternatively, Eq. (11) may be rewritten as

$$
\frac{d\left(V_{1}\right)}{d t}=\left[\frac{K_{2}}{K_{1}}\right] \frac{d\left(V_{s}\right)}{d t}-V_{r}
$$

Then, since the value of $V_{r}$ is known, Eq. (11') can be integrated immediately and a solution for $V_{s}$ from Eq. (10) will lead directly to a solution for $V_{1}$.

\section{Evaporator Feed Tank_Material Balance}

A schematic representation of a generalized evaporator feed tank and the associated flow streams is shown in Fig. 5. Sources of feed material include waste output from canyon operations (volumetric flow rate $V_{x}$, specific gravity $S_{x}$, salt mass fraction $X_{x}$, and sludge mass flow rate $\left.W_{s 1}\right)$ and the receiving tank recycle stream discussed above. A steam jet is used to transfor liquid volumes between tanks. Therefore, during transfer of the recycle volume from the receiving tank to the feed tank, the stream becomes slightly diluted. This transfer dilution is accounted for by the addition of water flow rate $V_{d}$ to the transfer stream. Since the water addition is a fixed percent of the recycle stream $V_{d}=F V_{r}$ holds where $F$ is a constant. The calculation of the canyon waste stream volume and composition
is explained below. 


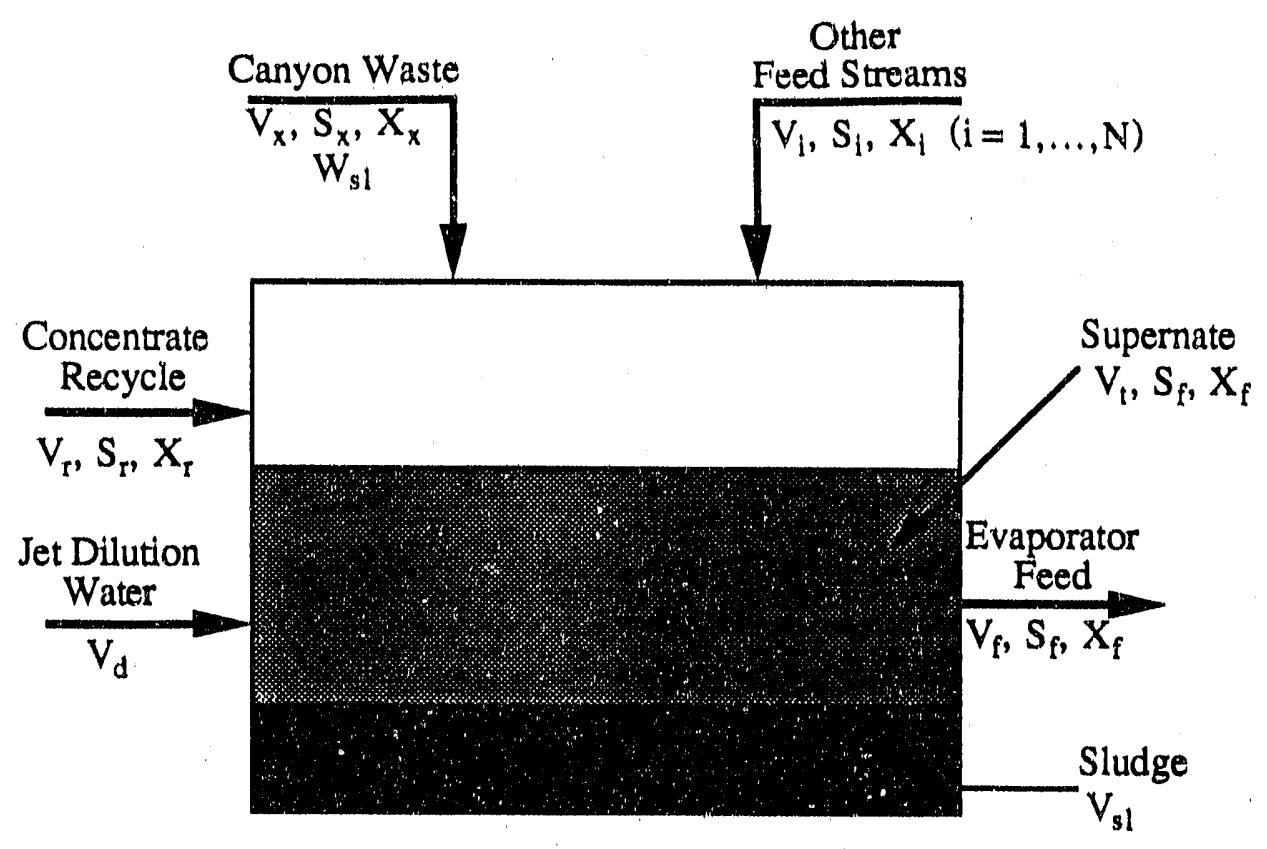

Figure 5. Evaporator feed tank flow schematic.

To generalize the model, additional sources of waste are included in the material balance calculations. That is, other waste streams of known flow rate and salt composition may enter the evaporator feed tank. For example, the sludge wash water and DWPF recycle waste streams to tank 43 shown in Fig. $1 \mathrm{~b}$ must be included. Other sources of waste input to the feed tank are indicated by the $V_{i}$ streams (volumetric flow rate $V_{i}$, specific gravity $S_{i}$, and salt mass fraction $X_{i}$ ) in Fig. 5. There may be several additional sources of waste volume. To simplify the notation, without loss of generality, these other sources are carried through the following equation development as a single term.

Evaporator feed is removed from the tank at flow rate $V_{f}$. The model assumes a well mixed tank so that the feed specific gravity $S_{f}$ is equal to the specific gravity of the tank liquid. The volume of liquid waste in the feed tank $V_{t}$, the liquid specific gravity $S_{f}$, and salt mass fraction $\mathrm{X}_{\mathrm{f}}$ are all functions of time as waste material is mixed into and removed from the tank.

Instantaneous material balance equations for total material and total salt in the feed iank may then be written in terms of specific gravity as

$$
\frac{d\left(V_{t} s_{f}\right)}{d t}=V_{x} s_{x}+V_{r} s_{r}+V_{i} s_{i}+F V_{r}-V_{f} s_{f}
$$

and

$$
\frac{d\left(V_{t} s_{f} x_{f}\right)}{d t}=V_{x} s_{x} x_{x}+V_{r} s_{r} x_{t}+V_{i} s_{i} x_{i}-V_{f} s_{f} x_{f}
$$

These equations represent the basic material balance equations for the evaporator feed tank. The subscript $x$ is used to denote canyon waste, $r$ indicates recycle material, $i$ other sources 
of waste, and $f$ denotes the evaporator feed stream. The notation may be simplified somewhat by introducing the terms

and

$$
Q_{1}=V_{x} S_{x}+V_{r} S_{r}+V_{1} S_{i}+F V_{r}
$$

$$
Q_{2}=V_{x} s_{x} X_{x}+V_{r} s_{r} X_{r}+V_{1} s_{i} X_{i}
$$

to obtain the equivalent expressions

$$
\frac{d\left(V_{t} S_{f}\right)}{d t}=Q_{1}-V_{f} S_{f}
$$

and

$$
\frac{d\left(V_{t} S_{f} X_{f}\right)}{d t}=Q_{2}-V_{f} S_{f} X_{f}
$$

The term $Q_{1}$ represents the total material mass inflow to the feed tank while $Q_{2}$ represents the total mass of salt inflow. In general, the quantities containing $V_{i}$ stand for the summation over several input streams.

All of the waste streams from canyon operations that enter the evaporator feed tanks will contain approximately $5 \%$ by volume sludge. The model assumes that the sludge component of the waste separates from the stream and is deposited directly into the feed tank. That is, the sludge is treated as a separate component of the waste stream from the salt-water solution.

\section{Solution of the Material Balance Equations}

Having established the appropriate algebraic and differential material balance equations for the evaporator system components, these equations will now be combined to produce a solution for the complete system. The equations can be solved analytically under very geniral conditions and this solution forms the basis for the computer simulation that was programmed.

Multiplying Eq. (12) by $X_{c}$ and subtracting Eq. (13) from the result gives the expression

$$
\begin{aligned}
\frac{d\left[V_{t} s_{f}\left(X_{c}-X_{f}\right)\right]}{d t}= & V_{x} s_{x}\left(X_{c}-X_{x}\right)+V_{r} S_{r}\left(X_{c}-X_{p}\right)+ \\
& V_{i} S_{i}\left(X_{c}-X_{i}\right)+F V_{r} X_{c}-V_{0} X_{c} \\
= & Q_{1} X_{c}-Q_{2}-V_{0} X_{c}
\end{aligned}
$$

In deriving Eq. (16), we have used Eq. (5), written as $V_{f} S_{f}\left(X_{0}-X_{f}\right)=V_{0} X_{c}$, to eliminate the feed tank variables from the right hand side.

For a particular evaporator system, the quantities $S_{\mathrm{r}}, \mathrm{X}_{\mathrm{r}}, \mathrm{X}_{\mathrm{c}}$, and $\mathrm{F}$ are known constants while the quantities $V_{x}, S_{x}, X_{x}, V_{r}, V_{0}, V_{i}, S_{i}$, and $X_{i}$ may experience discrete changes in their values. However; within known time periods (for example over one day), evaporator operating conditions remain constant and it can be assumed that all of the terms on the right hand side of Eq. (16) have fixed values. Therefore, in general, the equation may be 
integrated from a starting time to to a time $t$ during which the right hand side is constant to yield

$$
\begin{aligned}
V_{t} S_{f}\left(X_{c}-X_{f}\right) & =F_{0}+\left(Q_{1} X_{c}-Q_{2}-V_{0} X_{c}\right) \Delta t \\
& =F_{0}+C_{1} \Delta t
\end{aligned}
$$

where $F_{0}$ is the value of the function $\left[V_{t} S_{f}\left(X_{c}-X_{f}\right)\right]$ at the initial time $t_{0}$ and $\Delta t=t-t_{0}$ is the integration time interval. The combined term $\left(Q_{1} X_{c}-Q_{2}-V_{o} X_{c}\right)=C_{1}$ is also a constant over time interval $\Delta t$.

The left hand side of Eq. (13) may be rewritten as the sum $V_{t} S_{f} d\left(X_{f}\right) / d t+X_{f} d\left(V_{t} S_{f}\right) / d t$. Substituting Eq. (12) for $d\left(V_{t} S_{f}\right) / d t$, collecting terms, and using the definitions for the constants $Q_{1}$ and $Q_{2}$, then gives the result that

$$
V_{t} S_{f} \frac{d\left(X_{f}\right)}{d t}=Q_{2}-Q_{1} X_{f}
$$

When Eq. (17) is used to replace $\left(V_{t} S_{f}\right)$ in Eq. (18), an expression for the time rate of change of $\mathrm{X}_{\mathrm{f}}$ is derived

$$
\frac{d\left(X_{f}\right)}{d t}=\left(Q_{2}-Q_{1} X_{f}\right) \frac{\left(X_{c}-X_{f}\right)}{\left(F_{0}+C_{1} \Delta t\right)}
$$

After separation of variables, Eq. (19) is integrated and, with some algebraic manipulation of the result, the following expression for $X_{f}$ as a function of time is obtained where $\tau=\Delta t$

$$
X_{f}(t)=\frac{Q_{2} G(\tau)-X_{c} H(\tau)}{Q_{1} G(\tau)-H(\tau)}
$$

The functions of time $G(\tau)$ and $H(\tau)$ used to write Eq. (20) are defined as

and

$$
\mathrm{G}(\tau)=\mathrm{F}_{0}+\mathrm{C}_{1} \Delta \mathrm{t}
$$

$$
H(\tau)=F_{0}\left[Q_{2}-Q_{1} X_{f}(0)\right] \frac{\left[F_{0} / G(\tau)\right]^{p}}{\left[X_{c}-X_{f}(0)\right]}
$$

where $X_{f}(0)$ is the value of $X_{f}$ at time to and the power $p$ is equal to $\left(V_{0} X_{c}\right) / C_{1}$. To simplify Eq. (22), most of the constants can be collected into a single term $C_{2}$ defined by

$$
C_{2}=F_{0} \frac{\left[Q_{2}-Q_{1} X_{f}(0)\right]}{\left[X_{c}-X_{f}(0)\right]}
$$

Using this definition for $\mathrm{C}_{2}$, a simplified form of Eq. (22) is then written as 


$$
H(\tau)=C_{2}\left[\frac{F_{0}}{G(\tau)}\right]^{p}
$$

The feed tank liquid volume is calculated from Eq. (17), after using Eq. (1) to eliminate $S_{\mathrm{f}}$, as either

$$
V_{t}(t)=G(\tau) \frac{\left[1-K_{a} X_{f}(t)\right]}{\left[X_{c}-X_{f}(t)\right]}
$$

or

$$
V_{t}(t)=\frac{\left[\left(Q_{1}-K_{a} Q_{2}\right) G(\tau)-\left(1-K_{a} X_{c}\right) H(\tau)\right]}{\left[X_{c} Q_{1}-Q_{2}\right]}
$$

Equation (25) is derived from (24) by replacing $X_{f}(t)$ with the equivalent function of time from Eq. (20). The term in the numerator, $\left(Q_{1}-K_{a} Q_{2}\right)$, may be simplified by applying Eq. (1) to show that

$$
Q_{1}-K_{a} Q_{2}=Q_{3}=V_{x}+V_{r}+V_{i}+F V_{r}
$$

The constant $Q_{3}$ is equal to the total volumetric flow of waste into the evaporator feed tank. With this simplification, the feed tank liquid volume as a function of time is given by

$$
V_{t}(t)=\frac{\left[Q_{3} G(\tau)-\left(1-K_{a} X_{c}\right) H(\tau)\right]}{\left[X_{c} Q_{1}-Q_{2}\right]}
$$

The volumes of salt cake $\left[\mathrm{V}_{s}(t)\right]$ and liquid waste $\left[\mathrm{V}_{1}(t)\right]$ in the concentrate receiving tank are calculated by substituting the expression for $X_{f}(t)$ from Eq. (20) into Eq. (10) and integrating. After considerable algebraic manipulation and simplification of the result, the following expression is derived

$$
V_{s}(t)=V_{s}(0)+K_{1} J(\tau)
$$

where a new function of time defined as

$$
J(\tau)=\frac{\left[H(\tau)+\left(Q_{2} V_{0} \tau\right)-C_{2}\right]}{\left[X_{c} Q_{1}-Q_{2}\right]}
$$

has been introduced to simplify the notation. Equation (11') may be integrated directly and combined with the solution for $V_{s}(t)$ in Eq. (28) to obtain

$$
V_{1}(t)=V_{1}(0)+K_{2} J(\tau)-V_{r} \tau
$$

In Eqs. (28) and (30), $V_{s}(0)$ and $V_{1}(0)$ represent the values of $V_{s}$ and $V_{1}$ at time $t_{0}$, respectively. For computational convenience, it is useful to rewrite the constants $K_{1}$ and $\mathrm{K}_{2}$ in terms of only specific gravity values. Eliminating the salt mass fractions $\mathbf{X}_{\mathbf{s}}, \mathbf{X}_{\mathbf{c}}$, and $\mathrm{X}_{\mathrm{r}}$ from the definitions by using Eq. (1), the constants may be reformulated as 


$$
K_{1}=\frac{\left[S_{c}-S_{r}-R\left(S_{r}-1\right)\right]}{\left[S_{c}\left(S_{s}-S_{r}\right)\right]}
$$

and

$$
K_{2}=\frac{\left[S_{s}-S_{c}+R\left(S_{s}-1\right)\right]}{\left[S_{c}\left(S_{s}-S_{r}\right)\right]}
$$

With an expression for $X_{f}(t)$ available, Eq. (5) is solved directly for the evaporator feed rate $V_{f}(t)$ and Eq. $(6)$ is used to calculate the concentrate production rate $V_{c}(t)$.

The above analysis completes the treatment of an evaporator system. While the analysis has been quite general, certain special cases occur which must be handled separately. These special cases will now be developed.

\section{Treatment of 1F-2F Dual Evaporator System}

The F-Area low heat waste system must be treated in a slightly different manner since one feed tank serves two evaporators. In some instances, both the $1 \mathrm{~F}$ and $2 \mathrm{~F}$ evaporators will also use the same receiving tank. It is found that a slight modification of the basic equations developed above is sufficient to handle these differences.

Writing the material balance Eqs. (3) and (4) for each of the evaporators using a common feed tank gives

$$
V_{f}^{n} S_{f}=V_{o}^{n}+V_{c}^{n} S_{c}
$$

and

$$
V_{f}^{n} S_{f} X_{f}=V_{c}^{n} S_{c} X_{c}
$$

where $\mathrm{n}=1,2$ for the individual evaporators. As noted above, it is assumed that all of the waste evaporators produce concentrate of the same composition. Therefore, $\mathbf{S}_{\mathrm{c}}$ and $\mathrm{X}_{\mathrm{c}}$ are equal for both evaporators.

Generalizing the receiving tank material balance Eqs. (10) and (11) in the same way yields

$$
\frac{d\left(V_{s}^{n}\right)}{d t}=K_{1}^{n} V_{o}^{n}\left[\frac{X_{f}}{X_{c}-X_{f}}\right]
$$

and

$$
\frac{d\left(V_{1}^{n}\right)}{d t}=K_{2}^{n} V_{o}^{n}\left[\frac{X_{f}}{X_{c}-X_{f}}\right]-V_{r}^{n}
$$

The constants $K_{1}$ and $K_{2}$ are determined from Eqs. (31) and (32) and, in general, will be different for each evaporator from the dependence on $S_{8}(i)$. As before, an equivalent form of Eq. (36) is simply

$$
\frac{d\left(V_{1}^{n}\right)}{d t}=\left[\frac{K_{2}^{n}}{K_{1}^{n}}\right] \frac{d\left(V_{3}^{n}\right)}{d t}-V_{r}^{n}
$$


The differential material balance equations for the system feed tank are formulated as before to obtain

and

$$
\frac{d\left(V_{t} S_{f}\right)}{d t}=Q_{1}-\sum_{n=1}^{2}\left(V_{f}^{n} S_{f}\right)
$$

$$
\frac{d\left(V_{t} S_{f} X_{f}\right)}{d t}=Q_{2}-\sum_{n=1}^{2}\left(V_{f}^{n} S_{f} X_{f}\right)
$$

The term $Q_{1}$ again is the total mass inflow to the feed tank while $Q_{2}$ is the total mass of salt inflow. Generalized expressions for these inflow terms (including $Q_{3}$ ) are given by

$$
\begin{gathered}
Q_{1}=V_{x} S_{x}+\sum_{n=1}^{2}\left(V_{r}^{n} S_{r}^{n}\right)+V_{i} S_{1}+F \sum_{n=1}^{2}\left(V_{r}^{n}\right) \\
Q_{2}=V_{x} S_{x} X_{x}+\sum_{n=1}^{2}\left(V_{r}^{n} S_{r}^{n} X_{r}^{n}\right)+V_{i} S_{i} X_{i}
\end{gathered}
$$

and

$$
Q_{3}=V_{x}+V_{i}+(1+F) \sum_{n=1}^{2}\left(V_{r}^{n}\right)
$$

The only change from before is that the possibility of individual recycle streams of different composition must be accounted for. Over known time intervals, all of the terms comprising $Q_{1}, Q_{2}$, and $Q_{3}$ have constant values.

The above set of equations may be integrated exactly as before. Equation (37) is multiplied by $X_{c}$ and Eq. (38) is subtracted from the result. Equation (34) is then used to eliminate the terms $V_{f}^{n} S_{f}\left(X_{c}-X_{f}\right)$ to give

$$
\frac{d\left[V_{t} S_{f}\left(X_{c}-X_{f}\right)\right]}{d t}=Q_{1} X_{c}-Q_{2}-\sum_{n=1}^{2}\left(V_{0}^{n} X_{c}\right)
$$

Equation (42) is identical to Eq. (16) when the summation terms are combined using an effective overhead rate defined as

$$
V_{e}=\sum_{n=1}^{2}\left(V_{o}^{n}\right)
$$

Using the modified constants $Q_{1}, Q_{2}$ and $Q_{3}$, and the effective overhead rate $V_{0}$ in place of $V_{o}$, the solution of the system material balance equations is accomplished exactly as before. That is, Eq. (42) is integrated and the result combined with Eqs. (37) and (38) to derive 
analytical expressions for $\mathrm{X}_{\mathrm{f}}(\mathrm{t})$ and $\mathrm{V}_{\mathrm{t}}(\mathrm{t})$. These expressions are identical in form to Eqs. (20) and (27) but use the modified constant values to evaluate the functions. In particular, $\mathrm{C}_{1}=\left(\mathrm{Q}_{1} \mathrm{X}_{\mathrm{c}}-\mathrm{Q}_{2}-\mathrm{V}_{\mathrm{e}} \mathrm{X}_{\mathrm{c}}\right)$ and $\mathrm{p}=\left(\mathrm{V}_{\mathrm{e}} \mathrm{X}_{\mathrm{c}} / \mathrm{C}_{1}\right)$ must be used.

Using the analytical expression for $X_{f}(t)$, Eq. (35) is integrated to determine $V_{s}^{n}(t)$ as

$$
V_{s}^{n}(t)=V_{s}^{n}(0)+K_{1}^{n} \frac{V_{0}^{n} J(\tau)}{V_{e}}
$$

and Eq. $\left(36^{\prime}\right)$ is used to derive

$$
V_{1}^{n}(t)=V_{1}^{n}(0)+K_{2}^{n} \frac{V_{0}^{n} J(\tau)}{V_{e}}-V_{r}^{n} \tau
$$

For use in Eqs. (43) and (44), the function of time $J(\tau)$ must now be evaluated as

$$
J(\tau)=\frac{\left[H(\tau)+\left(Q_{2} V_{e} \tau\right)-C_{2}\right]}{\left(X_{c} Q_{1}-Q_{2}\right)}
$$

The more general treatment developed here will correctly model the dual $1 \mathrm{~F}-2 \mathrm{~F}$ evaporator system. When both evaporators use the same receiving tank, the total accumulation of salt and liquid waste volume in the tank will be correctly calculated by summing the individual contributions from each evaporator using Eqs. (43) and (44).

\section{Special Case of No Input to Evaporator Feed Tank}

A special situation occurs when there is no material flowing into the feed tank. In this case, the analysis presented above must be modified to correctly calculate the material balances. With no flow of material into the feed tank, the quantities $Q_{1}, Q_{2}$, and $Q_{3}$ are identically zero which causes the denominators in Eqs. (27) and (29) to vanish. A simplified analysis can be used to alleviate this numerical problem.

The feed tank specific gravity will clearly remain constant when there is no material inflow. Equation (17) is then used to obtain the feed tank volume as a function of time directly as

$$
V_{t}(t)=V_{t}(0)-\frac{V_{0} X_{c} \tau}{\left[S_{f}\left(X_{c}-X_{f}\right)\right]}
$$

where $S_{f}=S_{f}(0)$ and $X_{f}=X_{f}(0)$ are known constants. The $V_{0}$ in Eq. (46) can be replaced by $V_{B}$ for the dual evaporator case; however, the analysis will be restricted to a single evaporator to simplify the development and notation.

Equations (5) and (6) show that the evaporator feed rate and concentrate production rate have constant values given by

$$
V_{f}=\frac{V_{o} X_{c}}{\left[S_{f}\left(X_{c}-X_{f}\right)\right]}
$$


and

$$
V_{o}=\frac{V_{o} X_{f}}{\left[S_{c}\left(X_{c}-X_{f}\right)\right]}
$$

Equation (10) is then integrated to directly obtain the volume of salt cake in the receiving tank in terms of $X_{f}$ as

$$
V_{s}(t)=V_{s}(0)+K_{1} \frac{V_{0} X_{f} \tau}{\left(X_{c}-X_{f}\right)}
$$

Equation (11) is integrated, with $\mathrm{V}_{\mathrm{r}}$ equal to zero, to obtain an expression for the liquid volume in the recelving tank as

$$
V_{1}(t)=V_{1}(0)+K_{2} \frac{V_{0} X_{f} \tau}{\left(X_{c}-X_{f}\right)}
$$

This set of equations is used to calculate system parameters when there is no flow of material into the feed tank.

\section{Special Case When the Evaporator is not Functioning}

The equation set developed above is found to work correctly for the limiting case when the evaporator is not functioning. In this situation, the overhead rate $V_{0}$, or $V_{B}$, is identically zero. The evaporator and concentrate flow rates $V_{f}$ and $V_{c}$ will then vanish as required by Eqs. (5) and (6).

When $V_{0}=0$, the exponent $p$ in Eq. $\left(22^{\prime}\right)$ is also zero and $H(\tau)$ evaluates to $C_{2}$. Substituting for $\mathrm{H}(\tau)$ into Eq. (29) shows that $\mathrm{J}(\tau)$ is then identically zero. Therefore, Eqs. (28) and (30) may still be used to calculate the receiving tank salt and liquid volumes, $V_{s}(t)$ and $V_{1}(t)$, respectively, with the correct results. Equation (27) may also be used to determine the volume of liquid in the feed tank, $V_{t}(t)$, without modification.

\section{Treatment of Variable_Receiving Tank_Specific_Gravity}

The above analysis assumes that the supernatant liquid in the concentrate receiving tank is in equilibrium with salt cake. This implies that the specific gravity of the supernatant remains constant at the given value specified for each system. In certain situations this assumption is not correct.

For example, in the H-Area low heat waste system, before tank 38 was used as a concentrate receiving tank for the $2 \mathrm{H}$ evaporator, it contained a significant volume of lower specific gravity liquid. As part of the retirement program for type IV waste tanks, liquid waste from the older tanks was transferred into tank 38 . As the evaporator capacity permitted, this liquid was transferred from tank 38 to tank 43 for $2 \mathrm{H}$ evaporator feed. When tank 41 was completely filled with salt cake, evaporator concentrate was then sent to tank 38. Initially, the concentrate mixed with the lower specific gravity liquid present in the tank. After the liquid specific gravity reached the equilibrium value, salt cake began to form. 
Similarly, tank 30 in the high heat waste system is initially used as a holding tank for fresh high heat waste prior to evaporation. When the other receiving tanks are full (or the $1 \mathrm{H}$ evaporator starts up), tank 30 will be used as a concentrate receiving tank. At this time, the tank will contain some volume of liquid at a specific gravity less than that of salt cake supernatant. This liquid will mix with concentrated waste from evaporator operation and salt cake will not start to accumulate until the liquid specific gravity reaches the set equilibrium value.

It was advantageous to treat i.te variable receiving tank specific gravity in the following approximate manner. If the specific gravity is not equal to its equilibriurn value, it is nevertheless assumed to remain constant over one time step (one day) of system operation and salt cake is formed. During this time, if tank recycle occurs, it will take place at the current specific gravity. As developed above, the solution to the material balance equations requires a constant recycle specific gravity. Between time steps, the salt cake is redissolved until the supernatant liquid reaches the equilibrium specific gravity. At this point, the calculations can proceed as before. Actually the salt cake never forms while dilute concentrate is present and the model will correctly show this behavior using the procedure described above.

The correction process is illustrated schematically in Fig. 6. The following general material balances around the receiving tank give the basis for the correction calculations. In terms of specific gravity, an overall tank material balance is

$$
V_{1}^{i} s_{1}^{i}+V_{s}^{i} s_{s}=V_{1}^{f} s_{1}^{f}+V_{s}^{f} s_{3}
$$

where $V_{1}$ and $S_{1}$ are the liquid volume and specific gravity, respectively, $V_{s}$ and $S_{s}$ are corresponding salt cake values, and the superscripts $i$ and $f$ are used to indicate initial and final tank conditions.

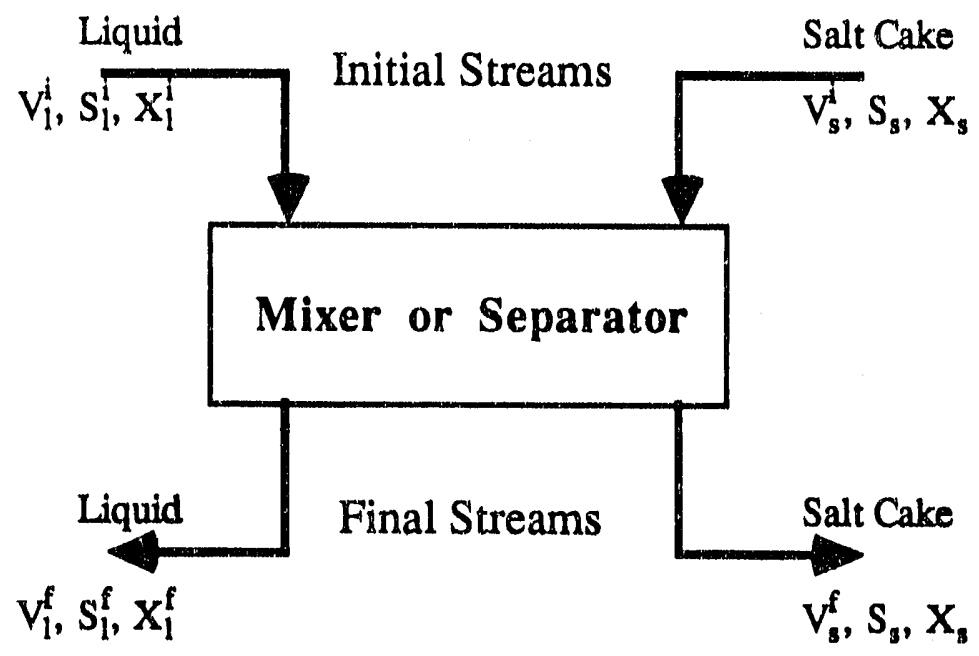

Figure 6. Schematic representation of mixing or separating a salt solution.

A material balance on the tank salt content gives 


$$
V_{1}^{i} s_{1}^{i} X_{1}^{i}+V_{s}^{i} s_{s} X_{s}=V_{1}^{f} s_{1}^{f} X_{1}^{f}+V_{s}^{f} s_{s} X_{s}
$$

Using Eq. (1) to eliminate the products of specific gravity and salt weight fraction in Eq. (52) and adding the result to Eq. (51) yields

$$
V_{1}^{l}+V_{s}^{l}=V_{1}^{f}+V_{s}^{f}
$$

Equation (53) indicates that material volumes are conserved under the assumption of isothermal ideal solutions used in the model.

The specific gravity correction may then be formalized as:

(1) Test the final tank liquid specific gravity $S_{1}^{f}$ with all of the salt cake dissolved, $V_{s}^{f}=0$. That is, using Eqs. (51) and (53) evaluate

$$
S_{1}^{f}=\frac{\left[V_{1}^{i} s_{1}^{i}+V_{s}^{i} s_{s}\right]}{\left[V_{1}^{i}+V_{s}^{i}\right]}
$$

(2) If the value of $S_{1}^{f}$ calculated from Eq. (54) is less than or equal to the equilibrium specific gravity $\left(S_{e}\right)$, then dissolve all of the salt cake present and assume that the tank specific gravity remains at $S_{1}^{f}$ for the next time step.

(3) If the value of $S_{1}^{f}$ calculated from Eq. (54) is greater than $S_{e}$, then set $S_{1}^{f}=S_{e}$ and partially dissolve (or precipitate) salt cake to balance the tank. Equations (51) and (53) are then solved for $V_{1}^{f}$ and $V_{s}^{f}$. The final liquid volume in the tank is given by

$$
V_{1}^{f}=V_{1}^{i} \frac{\left(S_{s}-S_{1}^{i}\right)}{\left(S_{s}-S_{e}\right)}
$$

(4) Once the tank liquid specific gravity reaches $S_{e}$, the testing procedure may be terminated and the calculations proceed as before with the supernatant and recycle specific gravity equal to $S_{e}$.

Model estimates of the receiving tank liquid specific gravity and recycle salt content at each time step may be slightly in orror with this treatment. If concentrate is mixing with the tank liquid when salt cake is not present, the specific gravity actually will be continuously changing. However, the overall error introduced into the model simulations by this approximate treatment is negligible. This treatment conserves total mass and salt mass while somewhat simplifying the computer programming. Use of a one day time increment for the model calculations is sufficiently accurate for the long term predictions of tank farm operating characteristics that are of most interest.

To summarize the above model development, the general material balance equations that were derived are presented as a complete set in the Appendix to this report. In writing the 
computer program that implements the model (WATFAM) an effort was made to comment the code for internal documentation. The equations presented in this section and the Appendix may then be compared to the FORTRAN listing to trace the flow of the simulation calculations.

\section{EVAPORATOR SYSTEM OPERATING RULES}

In addition to solving the material balance equations, a realistic simulation of the waste tank farm evaporator systems must conform to certain operating rules that constrain the system function. Operating rules specify when waste transfers occur, when concentrate recycle takes place, when receiving tanks are switched, and when the evaporator is in operation. A discussion of these operating rules is provided in this section.

A general rule in the simulation identifies tank overflows when the waste volume exceeds the operating limit by more than $10 \mathrm{kgal}$ or 3 inches of tank level. If any tank volume exceeds this limit the simulation is stopped and an error message is written to identify the problern. Although tank filling usually stops before the operating limit is reached, since the simulation uses one-day time increments, it is difficult to stop tank filling exactly at this level. Allowing the $10 \mathrm{kgal}$ excess volume permits the model to more closely approach actual tank operating conditions.

\section{Concentrate Receiving Tanks}

Concentrate receiving tanks are used until one million gillons of salt cake accumulate in the tank. At this point, the supernate is no longer recycled from the tank but concentrate feed to the tank is continued until the maximum operating volume is reached. Some amount of salt cake continues to precipitate out giving a final solids volume slightly in excess of 1000 kgal.

To prevent overfilling a receiving tank, the concentrate volume that will be added during the next day of operation is estimated by calculating $V_{c}$ from Eq. (48) at the start of the day. If the concentrate volume is greater than $80 \%$ of the remaining tank volume and the tank has accumulated all of the salt cake that it can hold, the receiving tank is removed from service and the next tank on the list used. The $80 \%$ factor was chosen to provide a conservative margin of error in tank volume usage. In practice, actual operations will be able to more fully utilize the tank capacity.

When a fresh receiving tank is required, it is possible that salt removal from the tank has not been completed. If this occurs, the model is programmed to write a message in the evaporator LOG file identifying the problem and to wait for the salt removal to finish. That is, evaporation will be stopped until concentrate can be sent to an empty receiving tank. If the feed tank overflows before evaporation can begin, a message identifying the problem is written in the LOG file and the simulation is stopped.

The opposite problem, that salt removal begins in a receiving tank still being used by an evaporator, could also occur. If this situation develops, the program will delay all remaining salt removals from the tank by four months. A message is written to the evaporator LOG file indicating that the salt removal schedule needs to be revised. For most cases, particularly the high heat waste systems, there must be a significant delay between filling the receiving tank with salt cake and starting salt removal. The model does not automatically delay salt removal after a tank is filled but will simply try to follow the input removal schedule as closely as possible. 
The model program keeps track of the salt cake and waste volume removed from the receiving tanks and stores this data for later retrieval.

\section{Evaporator Operation}

If evaporation would exhaust the supply of feed material, the evaporator must be stopped. The evaporator feed volume is estimated by calculating $V_{f}$ from $E q .(47)$ at the start of each time step. The avallable feed material is determined by adding an estimate of the incoming feed from Eq. (26) to the existing liquid volume in the feed tank. Only liquid volume above the minimum operating limit in the feed tank is considered to be avallable for evaporation. If the required volume of feed is greater than $80 \%$ of the avallable feed stock, evaporation is suspended.

To avoid frequent stopping and restarting, evaporation is not started again until the liquid volume in the feed tank is greater than $108 \mathrm{kgal}$ or the free space in the feed tank is less than $108 \mathrm{kgal}$. A message is written into the LOG file indicating that evaporation has stopped because the feed material is low and a second. message is written when the evaporator resumes operation.

\section{Low Heat Waste Sources}

As shown in Figs. $1 \mathrm{a}$ and $1 \mathrm{~b}$, there are several sources of waste for the low heat evaporators and rules governing the transfer of these materials into the evaporator systems are required in the model. During fiscal year 1986, a program to remove waste from old type IV tanks was in progress. In F-Area, this waste was transferred directly into tank 26 to feed the low heat waste evaporators. During part of the year, the waste was stored in tank 47 prior to evaporation. However, to simplify progiamming, the simulation sends liquid waste removal in F-Area to tank 26 since the error inherent in this treatment is small. In H-Area, liquid removed from old waste tanks was first transferred into tank 38 (this tank was not a concentrate receiving tank in 1986) and from there into tank 43 to feed the $2 \mathrm{H}$ evaporator. Logic for these transfers was included in the simulation.

The amount of liquid waste to be removed from type IV tanks and the specific gravity are input as parameters to the simulation. The user also specifies starting and ending dates for the transfer program which were assumed to be during FY 1986. The simulation model uses these waste volumes when space is available in the evaporator feed tanks. In H-Area, if adequate space is present in tank 43 , material is transferred from tank 38 . When tank 38 has at least $108 \mathrm{kgal}$ of available space, a transfer of waste removal material is made into the tank. When tank 38 is needed as a receiving tank, the removal of waste from type IV tanks in H-Area stops. If the evaporator systems are unable to process the total volume of waste removal in the specified time period, a message reporting the unused volume is written into the evaporator LOG file. The simulation is allowed to continue even if all of the waste removal volume was not processed. The model user must examine the LOG file to determine if the entire volume of waste removal liquid was processed. Processing of waste from old type IV tanks is given priority over processing sludge wash water in HArea and over transfers of material into tank 26 through the inter-area line in F-Area. In the future, waste volume generated from the retirement of older waste tanks in H-Area will go directly to tank 43 since tank 38 is now a salt tank. If it is desirable to include the processing of waste removed from old tanks in the model for years beyond 1986, some code revision is needed to correctly simulate this process. 
The recycle stream from the DWPF process shown in Fig. 16 is treated as a continuous source of waste material to evaporator feed tank 43 . This waste stream begins when the DWPF startup date is reached. Since this volume of liquid waste would eventually overfill tank 43, after the recycle stream is started, the model is programmed to transfer liquid volume from tank 43 to tank 26. The evaporator system in F-Area is expected to process the extra waste volume (note that currently the $1 F$ evaporator is not operating and it may not be restarted; therefore, the F-Area system is now reduced to a single evaporator). Workoff of the waste removal volume in F-Area is given priority over inter-area transfers. If a new $1 \mathrm{H}$ evaporator Is placed in service, excess waste volume in tank 43 is transferred into tank 32 for feed to the $1 \mathrm{H}$ evaporator instead of into tank 26.

When the free space in tank 43 is less than $216 \mathrm{kgal}$, the liquid volume becomes available for transfer to tank 26 over the inter-area line. An exact analydcal solution to the matertal balance equations could not be obtained when the evaporator feed tanks were coupled. Therefore, an approximate treatment was used. If a transfer from tank 43 to tank 26 is required, the material is instantaneously removed from tank 43 and is feed into tank 26 at a continuous rate the following day. Of course, the volume in tank 43 will not change immediately. However, daily changes in tank specific gravity and liquid volume are usually small and the error associated with an immediate change in the tank volume is negligible.

Another source of low heat waste is sludge wash water which will become avallable for evaporation in batches. To simulate the batch behavior, the amount of sludge wash water is increased by three months of processing volume each quarter. The volume of sludge wash water is accumulated and transferred into the $2 \mathrm{H}$ evaporator feed tank (43) as space becomes available. When the model is unable to transfer all of the sludge wash water by the end of the fiscal year, an error message giving the volume of unprocessed wash water is written into the $2 \mathrm{H}$ evaporator LOG file. The unprocessed volume is carried over into the next year and the simulation is allowed to continue. The starting date for sludge wash water should be at the beginning of a quarter (i.e month number $1,4,7$, or 10) to correctly include the first batch. Otherwise, sludge wash water is not added untll the start of the next quarter. The first batch contains three months of wash volume. The volume of sludge wash water processed through the evaporator is input to the code as $\mathrm{kgal} / \mathrm{month}$.

Sludge present in the canyon waste and in the DWPF recycle is accumulated in the feed tanks independently of the salt solution. That is, the sludge volume in tanks 26 and 43 is adjusted at each time step for inflow. When sludge removal is started, the volumes are decreased on the specified dates by the amount removed. In actual practice, evaporation will be stopped when the feed tanks undergo sludge removal. While this stoppage was not directly programmed, evaporator utilities for years with sludge removal can be lowered to account for this lost operating time. The simulation keeps track of the cumulative sludge removal from the evaporator systems for each fiscal year.

Other sources of waste volume and salt such as RBOF waste are accounted for through the miscellaneous waste stream. This additional waste material is specified through the input for each evaporator system and is added directly to the evaporator feed tanks.

\section{High_Heat Waste_Receipt Tanks}

Operating rules are required to control the flow of fresh high heat waste into the $1 \mathrm{H}$ evaporator system. As noted in the introduction (see Figs. 1b and Ic), most of the differences between the model as coded and actual tank farm operations occur in the HArea high heat waste evaporator system. Therefore, some of the modeling assumptions 
described here do not correspond to current operating practice. We have attempted to identify places where differences exist and note the changes needed to update the model.

Fresh canyon waste is stored in holding tanks and aged for at least one year before evaporation. To inltialize the simulation, the holding tank receiving fresh waste and the age of the other tank are specified for both $\mathrm{F}$ and $\mathrm{H}$ areas. The model feeds fresh waste in to the receiving tank until the tank is full. At that time, foed is switched to another holding tank and the aging process begins. When the waste has aged for one year, it is available as evaporator feed. Model simulations are sensitive to the timing of these waste transfers in the high heat system. If aged waste is not transferred out of a holding tank rapidly enough, when the other tanks are filled, a partially empty tank must be used to receive fresh waste. This can lead to situations where the tank refills before the other tank has aged sufficiently to be used as evaporator feed. At that point the holding tanks will overfill and the simulation stops. An error message explaining the problem is written into the $1 \mathrm{H}$ evaporator LOG file. The model uses two recelving tanks for high heat waste in both $\mathrm{H}$ and $\mathrm{F}$ area while current operations actually use three receivers in $\mathrm{H}$ area. Updating the model to incorporate this change may eliminate much of the overfilling problem.

To avoid overfilling as currently programmed, the simulation model tries to optimize the use of holding tank space. As soon as waste in a holding tank has aged for one year, it is transferred into the evaporator feed tank when space is available. When more than one tank has aged, the one containing the largest volume of liquid is emptied first. As explained above, the model assumes that tank 30 acts as an intermediate feed tank before being used as a concentrate receiving tank. The model transfers liquid waste from any of the F-Area and $\mathrm{H}$-Area fresh waste receipt tanks into tank 30 to use the available capacity. Tanks 30 and 35 then supply waste volume directly to the evaporator feed tank. Tank 35 is emptied in preference to tank 30 and, when tank 35 is empty or is receiving fresh canyon waste, the contents of tank 30 are feed into tank 13.

The model assumes that when tank 30 is used as a concentrate receiving tank, it is replaced with tank 32. If the $1 \mathrm{H}$ evaporator is replaced with a new unit, it is further assumed that tank 13 is removed from service simplifying the situation. Then all of the F-Area and HArea fresh high heat waste holding tanks feed directly into tank 32 which in turn feeds the $1 \mathrm{H}$ evaporator. At every time step, waste is removed from the tank having the largest available liquid volume. Current operations, as shown in Fig. 1c, actually use tank 30 as a concentrate receiving tank and use tank 32 directly as a high heat waste receiver not as an intermediate storage tank as assumed by the model. A more correct simulation would send aged waste from any of the high heat waste receiving tanks in F-Area and H-Area directly to tank 13 which serves as the $1 \mathrm{H}$. evaporator feed tank.

Waste transfers between tanks in the high heat system (and in the low heat waste system) include a $4 \%$ water clilution to account for steam added during the jet transfer process. Fresh waste transfers from the canyons into the receipt tanks do not include this dilution factor. However, all subsequent transfers are assumed to take place by steam eduction and are corrected for dilution. Transfers from waste removal tanks containing sludge in $\mathrm{H}$-Area are by pump and therefore do not include the water dilution.

The transfer scheme for the high heat waste tanks outlined above appears to work quite well. Test simulations have run continuously for up to 20 years. If the waste volume becomes too large for the system to handle, the simulation stops and the problem is identified to the user. The sludge component of the canyon waste is accumulated in the fresh waste recelpt tanks independently of the salt solution. 


\section{Concentrate_Recycle}

The simulation program contains a set of operating rules that determine how supernatant liquid is recycled from the concentrate recelving tanks to the evaporator feed tank. To prevent overfilling the feed tank, recycle takes place only when the evaporator is operating. The simulation begins a recycle if at least $35 \mathrm{kgal}$ of supernatant liquid is available and either of the following conditions is met:

1) The avallable space in the receiving tank is less than twice the estimated volume of concentrate (calculated from Eq. (6)) that will be sent to the tank during the next time step, or

2) The available liquid in the evaporator feed tank is less than twice the estimated volume of feed (calculated from Eq. (5)) required for the next time step of evaporator operation.

The simulation recycles supernatant liquid when the receiving tank is nearly full (before it has accumulated $1000 \mathrm{kgal}$ of solids volume) or when the feed tank is nearly empty to keep the evaporator systems operating. If an evaporator stops operating or if the volume of supernatant liquid available for recycle becomes less than $35 \mathrm{kgal}$, concentrate recycling is stopped in that system. In actual tank farm operations, recycling would continue while an evaporator was out of service. However, the model can not anticipate waste volumes entering the system or judge the availability of space in the feed tank so we have elected to stop recycling while the evaporator is not operating to be certain that fresh waste transfers can be accommodated without overfilling the feed tank.

The $35 \mathrm{kgal}$ minimum was established as an amount that is not worth recycling by itself. Based on a liquid transfer rate of $75 \mathrm{gpm}$, the maximum volume that can be recycled in one day is $108 \mathrm{kgal}$. The amount of material recycled in one day is taken as the smaller of: 108 $\mathrm{kgal}$, the available supernatant volume, or the space in the feed tarik. The actual volume of recycle then depends on prevailing tank conditions and represents actual system operations to some degree. The total volume of recycle is tracked by the program and reported in the output data. Messages indicating the start and the end of a recycle are written into the evaporator LOG files.

Concentrate recycles are accomplished using a steam-jet eductor which adds approximately $4 \%$ by volume of water to the recycle stream. This additional volume is accounted for with the $F$ factor in Eqs. (14) and (26). That is, $F$ is set equal to 0.04 in evaluating the equations. Water volume added to the evaporator systems by line flushing is not included directly in the model. This volume could be accounted for by including it in the miscellaneous waste additions if a significant volume is added during some time period.

\section{Waste Transfers}

The simulation must also decide when to transfer waste from external sources into the evaporator feed tanks, Canyon waste, DWPF recycle, and miscellaneous waste volumes are added to the evaporator feed tanks at a constant rate each day. Other waste sources are transferred into the feed tank as tank capacity is available. These external sources are:

1) $1 \mathrm{H}$ evaporator - Liquid volume in the high heat waste receipt tanks and in tank 30 (note: tank 30 is no longer used to hold fresh high heat waste but it presently still functions in this capacity in the model simulation) and the liquid volume in tank 43 after DWPF recycle begins if a new $1 H$ 
evaporator is in service. Note that the model transfers excess DWPF recycle to tank 43 while present operating plans are to send the recycle directly to tank 32 if the new $1 \mathrm{H}$ evaporator needs to process it. The model would need to be revised to correctly simulate this operation.

2) $1 \mathrm{~F}$ and $2 \mathrm{~F}$ evaporators - Waste removal from old type IV waste tanks in F-Area and the liquid volume in tank 43 after DWPF recycle begins.

3) $2 \mathrm{H}$ evaporator - Liquid volume in tank 38 while waste rernoval from old type IV waste tanks in H.Area was in progress (now completed), RBOF waste, and sludge wash water.

To determine if sufficient space is available in the evaporator feed tank to make a waste transfer, the simulation compares the available tank space to the expected volumes of recycle and waste transfer. If the space is greater than the expected volume additions by some minimum amount, a waste transfer is made. A minimum residual volume of $35 \mathrm{kgal}$ is maintained in the feed tank to allow space for canyon and miscellaneous waste additions. If the free space in the concentrate receiving tank becomes less than $108 \mathrm{kgal}$ and liquid is available for recycle, indicating that recycle will be required in the near future, the minimum volume retained in the feed tank is increased by $108 \mathrm{kgal}$. This is a conservative operating plan since it does not take credit for the expected volume reduction in the tank from evaporator feed.

Some waste transfers are effected by using steam-jet eductors which add approximately $4 \%$ by volume of water to the transfers. DWPF recycle and sludge wash water transfers have this additional $4 \%$ water volume included. Waste transfers from tank 38 to the evaporator feed tank in the $2 \mathrm{H}$ system include the $4 \%$ dilution. Transfers of waste from the old type IV tanks are accomplished by pumping and therefore do not include steam dilution. Likewise, fresh canyon transfers and additional waste input to the evaporator feed tanks are assumed to go directly into the tanks without steam dilution. The inter-area line (IAL) uses pumps not jets; however, transfers over the inter-area line include a $4 \%$ water dilution to account for the dilution during transfers from the waste tanks to the IAL pump tank which are accomplished with jets.

The simulation program keeps track of the total waste transfers made each fiscal year. Waste volumes removed from old type IV tanks in each area and the total sludge wash water processed each year are tracked separately. If the simulation is unable to completely process these waste volumes, error messages are written to the evaporator LOG files. Total transfer volumes are accumulated in the output data.

The model also tracks and reports the number of days that the inter-area transfer line is used. Transfers of high heat waste from F-Area to H-Area and waste transfers from tank 43 in H-Area to tank 26 in F-Area must be made over the inter-area line. The number of such transfers is reported in the LOG files at the end of each fiscal year. The model does not try to schedule the inter-area transfers but assumes that the line is available for all of the required transfers to take place.

\section{DETERMINATION OF CANYON WASTE GENERATION}

The principal source of fresh waste input to the tank farm evaporator systems is from $\mathrm{F}$ and $H$ area canyon operations. It is assumed that the waste generation can be calculated as a fixed monthly arnount plus an amount dependent on canyon material processing. For each waste type, the total volumetric generation $\left(V_{x}\right)$ is then calculated as 


$$
\mathrm{V}_{\mathrm{x}}=\mathrm{V}_{\mathrm{n}}+\mathrm{M} \mathrm{V}_{\mathrm{m}}
$$

where $V_{n}$ is a fixed volume of canyon waste generated irrespective of production, $M$ is the amount of material processed in canyon operations, and $V_{m}$ is the volume of waste generated from the processing of material $\mathrm{M}$. For F-Area canyon operations, $\mathrm{M}$ is equal to the metric tons of uranium processed, while for $\mathrm{H}$-Area canyon, $\mathrm{M}$ is equal to the number of reactor tubes processed.

To generalize the model treatment, the quantities $V_{n}, V_{m}$, and $M$ are input as parameters from the tank farm data base. Salt and sludge concentrations in the canyon waste streams are also determined from input parameters. A factor giving the weight of salt in each gallon of waste is specified. The total salt input is then converted into a specific gravity for the canyon waste stream using Eq. (1) and the relationship $W_{s}=V_{x} * S_{x} * p_{w} * X_{x}$ where $W_{s}$ is the weight of salt and $\rho_{w}$ is the density of water. Similarly, a factor to calculate the volume of sludge produced from the amount of material processed in canyon operations is input. Each of tisje parameters is specified separately for the high and low heat waste streams in both F-Area and H-Area. Examples of canyon waste parameters are presented with the tank farm data base discussed below.

The amount of material processed in canyon operations is also an input parameter for the model simulations. The model data base is set to accept the current Materials Management Plan (MMP) forecast as input data. The MMP forecast estimates the metric tons of uranium and the number of reactor tubes that will be processed in the separations areas. This forecast is usually on a monthly basis for the current fiscal year and on a yearly basis thereafter. As explained below, in the discussion of program WTFD, the model is programmed to accept data in this format and determine daily waste production from canyon operations by the method outlined above.

In effect then, a simple model of canyon waste generation, given in Eq. (56), has been included in the simulation. This removes specific details of canyon operations from the simulation and predicts canyon waste generation directly from the MMP forecast. If extra waste generation is anticipated, it can be included in the additional source term represented by $V_{i}$ in Eqs. (14) and (15) or the canyon parameters can be easily modified.

\section{WASTE TANKS OUTSIDE OF EVAPORATOR SYSTEMS}

In addition to the detailed model of the tank farm evaporator systems described above, the simulation includes simple models for some waste tanks not directly coupled to the evaporators. These are tanks 21 and 23 associated with the RBOF process, tanks 40, 42, and 51 used for sludge processing, and tanks 48,49 , and 50 used for the in-tank salt precipitation process.

Tanks 21 and 23 are modeled in a particularly simple fashion. Since the RBOF process is in operation and is expected to continue essentially unchanged in the future, wase volumes in these tanks are kept constant at the initially given values. The waste generated from RBOF operations is added to tank 43 by including its volume with the miscellaneous waste additions. RBOF processing adds approximately $120 \mathrm{kgal} /$ year to the $2 \mathrm{H}$ evaporator system [1]. This volume can be varied for each specified time period through the input.

The volume of waste in tanks 40,42 , and 51 is connected to the sludge removal schedule input to the model simulation. As sludge is removed from waste tanks, it is added to the 
three processing tanks. Sludge additions are made at the start of each month. Each tank is allowed to accurnulate $500 \mathrm{kgal}$ of sludge and $200 \mathrm{kgal}$ of additional liquid waste from the sludge transfers and processing operations. It is assumed that the tanks do not overfill during this operation so that if the tank volume reaches its maximum capacity it is held fixed at this level. When DWPF processing begins, $24.17 \mathrm{kgal}$ of sludge is removed from the sludge processing tanks each month as feed to the glass melter. The liquid volume in the tanks is then adjusted to be $40 \%$ of the sludge volume. Although using this simple model may not predict the waste volumes in individual tanks correctly, the overall sludge volume in the three tanks should track actual operating conditions with reasonable accuracy.

Waste volumes in the salt precipitation processing tanks $(48,49$, and 50$)$ are based on the salt removal schedule. As salt cake is removed from waste tanks, liquid volume is added to tanks 48 and 50 until $500 \mathrm{kgal}$ have accumulated in both tanks. Once they are filled to this level, the in-tank precipitation process will keep these tanks at a relatively constant volume. Tank 49 is reserved for precipitate holding. This process is modeled by assuming that 0.267 of the volume of salt cake removed from waste tanks appears as precipitate in tank 49. Excess liquid in tank 49 is removed to prevent overfilling while the precipitate accumulates. When $1000 \mathrm{kgal}$ of precipitate accumulate in tank 49 the volume is held constant if DWPF processing has started. If over $1000 \mathrm{kgal}$ of precipitate accumulate in the tank before DWPF processing starts, the tank will overfill and the simulation will stop.

The model described above to simulate the sludge and salt processing was based on information that was current as of 1988. It is possible that these processes will be modified as they are developed in the future. If modifications occur, some coding changes will be required to update the simulation.

\section{MODEL COMPUTER PROGRAMS}

The computer model to simulate operation of the waste tank farm is contained in a set of three separate programs. The structure and operation of these programs and the model data are explained in this section. Examples of model calculations and the output data generated are presented. A more complete description of the instructions needed to run these programs is provided in the Appendix.

\section{WTED - Tank Farm Data Base}

The first program in the model set is WTFD.BAS which is used to create and modify the tank farm data base that provides input for the model simulations. Since the data base is fairly large, it is convenient to separate the creation and writing of the data from the model calculations. The WTFD program is designed to facilitate entry and modification of model input data. The program is written in BASIC and can be run either as a compiled BASIC application or through the BASIC interpreter. Input data is contained in a file named TFARM.DAT. A partial listing of a typical data file illustrating the various types of data entry required is provided in the Appendix. The listing shows pages of the data as they appear on the computer screen when program WTFD is run. Parameter values given in the listing are representative of those used in actual simulations.

The first page of data (Fig. A4) is used to input the year and month when the following process functions occur:

1. Simulation starting date.

2. The date to begin waste removal from type IV tanks. 
3. The date to stop waste removal from type IV tanks.

4. A date when the F-Area canyon evaporator is changed.

5. A date when the $1 \mathrm{H}$ evaporator upgrade is completed.

6. The date to start the sludge wash water stream.

7. DWPF starting date.

All of these dates may be varied to test the effect of start-up timing on tank farm operations. The processes will begin on the first day of the specified month. For example, the data in Fig. A4 will start the simulation on October 1, 1986. Most of the waste has now been removed from the type IV tanks and dates 2 and 3 should be set prior to the starting date to avoid processing waste from type IV tanks.

Following the process dates, initial conditions for the 51 waste tanks in the tank farm are entered (Fig. A5). The tank fill limit is the maximum operating level for the tank and the jet elevation is the minimum level that is accessible in the tank. These values and the conversion between inches of tank level and gallons of tank volume will remain constant for each waste tank. Other input data will vary with starting date from continuing tank farm operations. The tank level in inches for salt, sludge, and total volume on the starting date and the specific gravity of the tank supernatant liquid are required input. Current data may be obtained from monthly tank farm inventory records.

The next set of input data (Fig. A6) specifies operating parameters for the four tank farm evaporators. The observed overhead production rates and lance steam addition rates are entered in gallons per day for each evaporator. The net evaporation rate is the difference between these two numbers. The basis specific gravity is used with Eq. (7) to determine a value of $\mathrm{K}_{e}$ for each evaporator from

$$
\mathrm{K}_{\mathrm{e}}=(\text { Overhead }- \text { Lance })+7560 *(\text { Basis SPG })
$$

With $\mathrm{K}_{e}$ determined, the net evaporator overhead rate is then predicted from Eq. (7) at each time step using the feed tank specific gravity at the start of the step. The flush water ratio $\left(R\right.$ in Eqs. (31) and (32)) and the recycle specific gravity $\left(S_{r}\right)$ are both evaporator dependent input parameters entered on this data page. The feed tank to be used for each evaporator is also specified here.

It is anticipated that the $1 \mathrm{H}$ evaporator will be replaced with a new unit in FY 1994. Therefore, a provision is made to change the $1 \mathrm{H}$ operating parameters on the date specified on the first page of data (Fig. A4). New operating parameters for the $1 \mathrm{H}$ evaporator are entered in the last column of the page (Fig. A6). All of the evaporator parameters are switched to the new values except for the designated feed tank.

When the concentrate receiving tanks fill with salt cake, the evaporator must switch to another tank. The order in which tanks are used is input in the next set of data (Fig. A7). The first entry is used as the starting receiving tank. A zero entry indicates that the schedule was not developed beyond this point. The simulation will stop when a zero entry is encountered. Since several years are required to fill a receiving tank with salt, a schedule allowing up to 10 entries should be sufficient for 20 year simulations.

For the $1 \mathrm{H}$ and $2 \mathrm{H}$ evaporator systems, the receiving tank schedules are straight forward. However, the $1 \mathrm{~F}$ and $2 \mathrm{~F}$ evaporators may use the same receiving tank in some instances. For example, in 1986 it was expected that the $2 \mathrm{~F}$ evaporator would fill tanks 47,45 , and 44 with salt cake by early 1987 . When this occurred, the $1 F$ and $2 F$ evaporators were both going to use receiving tank 27 and, when tank 27 was filled, both evaporators would next switch to tank 28 and then to tank 25 . It was further planned that the $2 F$ evaporator would 
immediately switch over to use tank 44 as soon as salt cake was removed from this tank and it was once again available as a concentrate receiving tank. Rather than program special cases such as this, the simulation would have actually allowed the $1 \mathrm{~F}$ and $2 \mathrm{~F}$ evaporators to fill both tanks 25 and 44 together before they began using separate schedules. Therefore, the rate at which tanks 25 and 44 filled with salt cake may not have be accurately predicted by the model but the overall time involved would have been correct. In actual practice this scenario did not occur as the $1 \mathrm{~F}$ evaporator was shutdown and the original receiving tanks are still not quite full of salt cake.

The next set of data inputs parameters for the low and high heat waste streams from $F$ and $\mathrm{H}$ area canyons (Fig. A8). It was expected that the F-Area high heat waste production would change during 1986 when a new canyon evaporator was placed in service. Therefore, two sets of data are provided for this waste stream and the second values are used after the daie given on the first data page (Fig. A4). The second entry is unnecessary at this time but the programs have not as yet been modified to eliminate this extra input. The first parameter for each stream is the fixed volume of waste generated by the canyons each month independent of the monthly production. The second data value is the volume of waste that is directly a function of canyon production. This number is multiplied by the number of tubes processed in H-Area or the metric tons of uranium processed in F-Area to give a total monthly waste volume. These volumes are $V_{n}$ and $V_{m}$ of Eq. (56), respectively. The last two parameters specify the salt and sludge content of the canyon waste streams. The amount of salt in the waste is found by multiplying the third parameter by the total volume of waste. It is assumed that sludge is only generated during canyon production and the volume of sludge is obtained as the product of the last parameter and the production units.

Production units and evaporator utilities are specified in the next set of data values (Fig. A9). For H-Area the number of reactor tubes processed over the next month or year are input and for F-Area the metric tons of uranium to be processed are specified. The production data is obtained from the current MMP forecast. A fractional utility is also entered for each of the four evaporators. This utility specifies evaporator availability during the indicated time period and is used in the simulation to assign evaporator down-time. For example, if a utility of 0.5 is specified, the simulation randomly selects one half of the days in the time period as days when the evaporator is not available for operation.

The time periods for data entry in this part of the program depend upon the starting date. From the given starting month until the end of the first fiscal year, monthly data is entered. In subsequent fiscal years, data entry is on a yearly basis. This change is illustrated in the lower half of Fig. A9. Space is provided for a total of 31 data values. That is, when the starting month is October, 12 monthly values followed by 19 yearly values are entered for the MMP forecast and evaporator utility data. When the starting month is September, only one monthly data value is entered followed by 30 yearly values. Thus the program is set to run for a minimum of 20 years. This data entry scheme reflects the fact that forecasts are prepared on a fiscal year basis and it allows the model to be run for partial years.

The next block of data sets a schedule for the removal of salt cake from old waste tanks and from concentrate receiving tanks (Fig. A10). The salt removal schedule is determined by Waste Management Operations [1] based on salt composition and curie content. Salt cake from more than one tank is blended for precipitate processing. For each tank, up to 20 salt removals may be specified. The date for salt removal is entered using a YEAR+(MONTH/100) format followed by the volume of salt cake to be removed in kgal. The YEAR parameter is the last two digits of the calendar year. The value of YEAR is zero for calendar year 2000 . 
Since it may be desirable to vary the amount of salt cake removed from the receiving tanks, some mechanism to flag the end of a salt removal sequeuce and indicate that a tank is free to be reused as a cuncentrate receiving tank is required. This was accomplished by making the last digit of the final volume of salt cake to be removed some number other than 0 or 5 . To minimize the error in the amounts, one $\mathrm{kgal}$ was added to each ending salt removal volume. For example, for receiving tank 25 in Fig. A10, the 226 and $151 \mathrm{kgal}$ volumes indicate the end of two sequences of salt removal. The tank is then free to accumulate salt cake again after $8 / 1 / 91$ and $8 / 1 / 02$.

A receiving tank is considered filled when it has accumulated $1000 \mathrm{kgal}$ of salt cake. At this point, the tank is topped-off to the fill limit with concentrate. The supernatant liquid contains the equivalent of another $100 \mathrm{kgal}$ of salt cake. Therefore, in the salt removal schedule, the amount of salt cake removed is greater than the solidified salt cake in the tank by this extra $100 \mathrm{kgal}$. The model will not remove more salt cake from a tank than the amount that is actually present in the solids and supernatant liquid volumes.

The simulation immediately removes salt cake from a tank when the removal date is reached and no attempt is made to include a rate of salt removal in the calculations. That is, on the first day of the indicated month, the specified amount of salt cake is removed from the waste tank. Since the primary impact of salt removal on the simulation is in determining when receiving tanks can be reused, the last date in a salt removal sequence for concentrate receiving tanks should be the date when the tank is expected to become available. That is, the date when salt removal from the tank is completed should be entered.

In a similar fashion to the salt removal schedules, sludge removal schedules for waste tanks are entered in the next set of data (Fig. A11). Again a YEAR+(MONTH/100) format for the date and removal volumes in $\mathrm{kgal}$ are used. Since sludge removal will typically occur in a few batches, space for 10 entries for each tank have been allocated and data for two tanks are displayed on each page.

The next data set (Fig. A12) inputs waste volume and salt additions to each evaporator system from sources other than those shown on the process flow sheet. Data is entered for the same time periods as were used with the production and evaporator utility data. Some anticipated sources of this extra waste volume are given in the Table 1 on a yearly basis. The total additional waste volume and salt content are entered by the user.

Table 1. Sources of additional waste water to evaporators.

\begin{tabular}{|c|c|}
\hline F-Area & H-Area \\
\hline Catch Tank $21 \mathrm{kgal}$ & $299-\mathrm{H} 84 \mathrm{kgal}$ \\
100 Area Trailers $34 \mathrm{kgal}$ & Miscellaneous $32 \mathrm{kgal}$ \\
Miscellaneous $20 \mathrm{kgal}$ & \\
\hline
\end{tabular}

These volumes are added directly to the evaporator feed tanks at a constant rate and correspond to the $V_{i}$ terms in Eqs. (14), (15), and (26). In the example data, salt addition is included by entering a stream specific gravity $\left(S_{i}\right)$ greater than one. This part of the data set can be used to test the impact of additional volumes of waste and salt on tank farm operations or to redistribute waste sources among the evaporator systems. The RBOF 
waste volume should be included in this data and sludge wash water and DWPF recycle volumes can be included to specify their distribution arid time dependence.

The final set of data includes several miscellaneous operating parameters (Fig. A13). As a part of the program to retire old Type IV waste tanks, some waste was removed from these tanks and fed directly into the low heat waste evaporator systems during fiscal year 1986. The first page of the last data set provides for input of the volurnes and specific gravities of these waste streams. The F-Area volume is fed directly to tank 26 while the H-Area volume first goes into tank 38 . The simulation will attempt to transfer the waste removal volumes into the evaporator systems between the dates specified in the first set of input parameters. These waste sources are given the lowest priority in the sense that the evaporator will only use them as capacity is available. These volumes are included as a component of the $V_{i}$ streams in the calculations. While it is not anticipated that these parameters will be needed in future simulations, they have been retained as a model feature and could possibly be used to simulate other waste streams.

Parameters for the DWPF recycle and sludge wash water streams are set in the next page of data. The first value gives stream flow rates in $\mathrm{kgal} / \mathrm{month}$. This is followed by the stream specific gravity which determines salt content and a sludge flow rate associated with the waste in $\mathrm{kgal} / \mathrm{month}$. When entered on this page, the DWPF recycle and sludge wash water are added directly to tank 43 as shown on the process flow sheet. As the example data indicates, these waste volumes can be set to zero and the waste included with the other additions discussed above to distribute the volume to other evaporator systems.

The final page of input data gives parameters for the fresh waste receipt tanks in the high heat waste system. The tanks receiving waste at the start of the simulation and the age of the waste material in the other tanks are entered. The example data in Fig. A13 indicates that fresh high heat waste from the H-Area canyon is being fed into tank 39 and that the waste in tank 35 has aged for 5 days when the simulation starts. Similarly in F-Area, fresh waste is being sent to tank 33 and the waste in tank 34 is 180 days old. The waste age is important since high heat waste must be aged for one year before evaporation. When the waste is 365 days old the tank volume becomes available for evaporator feed.

\section{WATFAM - Simulation Calculation}

Actual calculation of the tank farm simulation is performed by running the FORTRAN program WATFAM that encodes the mathematical model derived above. The screen display during execution of the WATFAM program is shown in Fig 7. Since simulation parameters are contained in the data base, input required to execute WATFAM is minimal. The program user only specifies the total number of days to run the simulation, the desired printout interval, a random number seed, and the type of log files desired. The program will stop when the input data is exhausted if too long a simulation is requested.

One feature of the WATFAM program is the random assignment of evaporator down-time. The random number input during program execution is used in conjunction with evaporator utilities to randomly assign days on which the evaporators are not operating. This introduces a stochastic element into the simulation making it possible to execute the program with the same data and variable random numbers to obtain different results. This feature could be used to determine the probability that the tank farm will experience problems for a given set of operating conditions. Running the model repeatedly while varying the random number would generate statistics on the number of tank farm failures with the specified parameters. A failure occurs when an evaporator system is unable to handle the waste input. Using this simulation feature would require a large number of 
separate computer runs. Running the simulation on a high speed computer would make this analysis feasible.

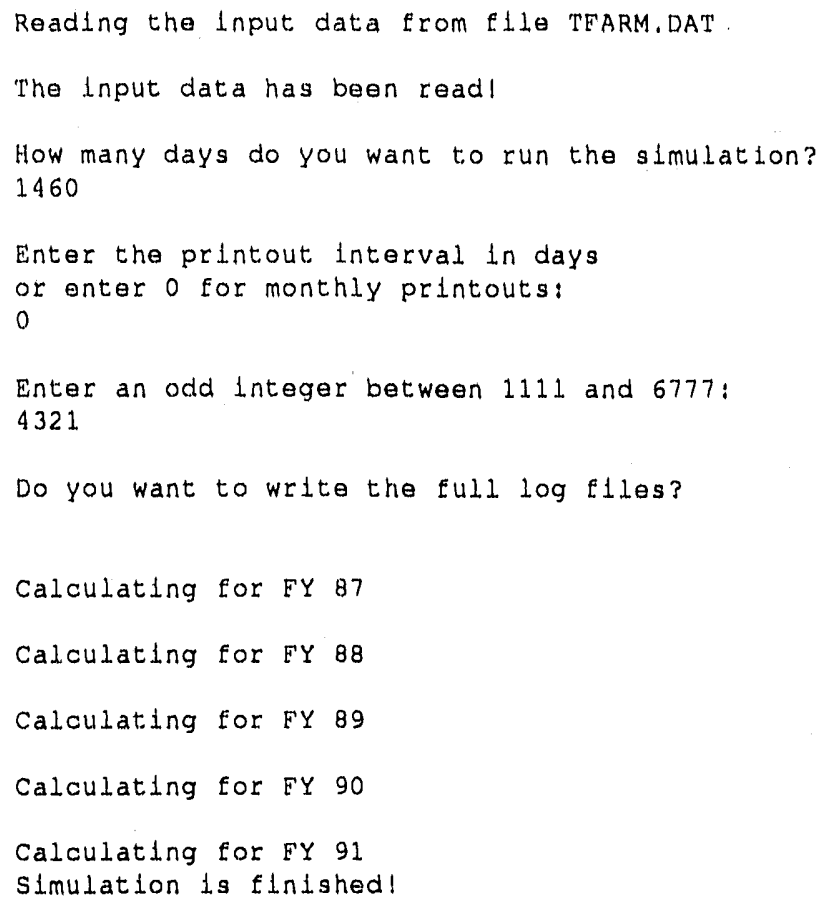

Figure 7. Screen display during a sample run of program WATFAM.

As the simulation runs, the fiscal year currently being computed is displayed on the screen so that the user is able to monitor the progress of the calculation. Output from WATFAM is stored in two data files TANK.DAT and EVAP.DAT and in log files for each evaporator system. The log files are ASCII files that can be accessed directly for reading and printing. The other output is in binary format and is read with the READER computer program.

A portion of the output from a typical log file, showing results from several months of operation for the 2F evaporator system, is presented in Fig. 8. This information can be used to determine exactly how the tank farm evaporator systems operated during the simulation. The timing of waste transfers and recycles and the random days on which the evaporator is not operating are given in the file. As shown in Fig. A8, a utility of $85 \%$ was specified for the $2 \mathrm{~F}$ evaporator in October 1986 which implies that the evaporator should not operate 5 days that month. The log file correctly shows that on October $3,12,18,27$, and 30 the evaporator was not operating. At the start of December 1986, a message is written indicating that tank 47 is full and tank 45 is now used as the receiving tank. At the bottom of Fig. 8, a message appears indicating that the evaporation is stopped on 10/8/87 because feed material in tank 26 is depleted as is resumed on 10/21/87 when sufficient feed stock has accumulated. 
10/1/86 start analysig of evaporator system $2 \mathrm{~F}$ 10/ 1/86 Begin to use recelving Tank 47 - FY 87 -

10/ 2/86 Recycle stream from Tank 47 to Tank 26 1s on. 10/ 3/86 Evaporator is not operating.

10/ 3/86 Reoyole stream from Tank 47 to Tank 26 ls off. 10/ 7/86 Recycle stream from Tank 47 to Tank 26 is on. 10/12/86 Evaporator is not operating.

10/12/86 Recycle stream from Tank 47 to Tank 26 Ls off. 10/18/86 Evaporator 1s not operating.

10/23/86 Recycle stream from Tank 47 to Tank 26 is on. 10/27/86 Evaporator is not operating.

10/27/86 Recycle stream from Tank 47 to Tank 26 ts off. 10/30/86 Evaporator is not operating.

-

12/ 1/86 Begin to use recelving Tank 45

12/ 2/86 Recycle stream from Tank 45 to Tank 26 is on.

12/ 7/86 Evaporator is not operating.

12/ 7/86 Recycle stream from Tank 45 to Tank 26 is off. 12/14/86 Evaporator is not operating.

12/19/86 Recycle stream from Tank 45 to Tank 26 is on. $12 / 23 / 86$ Evaporator is not operating.

12/23/86 Recycle stream from Tank 45 to Tank 26 is off. 12/25/86 Evaporator is not operating.

$12 / 29 / 86$ Evaporator is not operating.

5/ 1/87 Recycle stream from Tank 45 to Tank 26 is on. 5/ 3/87 Reoycle stream from Tank 45 to Tank $26 \mathrm{ls}$ off. 5/ 7/87 Evaporator is not operating.

5/ 9/87 Recycle stream from Tank 45 to Tank 26 is on. 5/11/87 Recycle stream from Tank 45 to Tank 26 1s off. $5 / 14 / 87$ Evaporator 1s not operating.

$5 / 20 / 87$ Evaporator is not operating.

$5 / 22 / 87$ Evaporator is not operating.

$5 / 24 / 87$ Begin to use recelving Tank 44

$5 / 26 / 87$ Recycle stream from Tank 44 to Tank 261 s on. $5 / 27 / 87$ Evaporator is not operating.

$5 / 27 / 87$ Recycle stream from Tank 44 to Tank 26 is off. $5 / 30 / 87$ Recycle stream from Tank 44 to Tank 26 is on. $5 / 31 / 87$ Recycle stream from Tank 44 to Tank 26 is off. -

- FY 88 -

10/ 3/87 Recycle stream from Tank 44 to Tank 26 is on.

10/ 4/87 Rucycle stream from Tank 44 to Tank 26 is off.

10/ 6/87 Recycle stream from Tank 44 to Tank 26 is on.

10/ 7/87 Recycle stream from Tank 44 to Tank 26 is off.

10/ 8/87 Evaporator is not operating.

$10 / 8 / 87$ Evaporator stops for low feed.

10/21/87 Evaporator resumes operation.

Figure 8. Partial listing of full $\log$ file for the $2 \mathrm{~F}$ evaporator system (2F.LOG) from a four year simulation. 
10/ 1/86 start analys 1 s of evaporator syatem 15

$10 / 1 / 86$ Begin to use reoelving Tank 27

- FY $87-$

- FY 88 -

- FY $89-$

- FY 90 -

$10 / 24 / 89$ Begin to use recelving Tank 28

.. FY 91 -

$10 / 1 / 86$ start analysis of evaporator system $1 \mathrm{H}$

10/ $1 / 86$ Begin to use recelving Tank 36

- FY 87 -

$3 / 19 / 87$ Begln to use recelving T'ank 37

9/30/87 Inter-area line requitred 9 days

- FY $88-$

4/ $1 / 88$ Beghn to use recalving Tank 30

- EY 89 -

9/30/89 Inter-area line required 8 days

- FY 90 -

$7 / 10 / 90$ Evaporator stops for salt removal.

- FY 91 -

$10 / 1 / 86$ start analys 1 s of evaporator system $2 \mathrm{~F}$

$10 / 1 / 86$ Begln to use recelving Tank 47

- FY 87 .

$12 / 1 / 86$ Begin to use reoulving Tank 45

$6 / 16 / 87$ Begin to use recolving Tank 44

- FY 88 -

$7 / 18 / 8$ Begin to use recelving Tank 27

- FY 89 -

- FY 90 -

$10 / 24 / 89$ Begin to use rededving Tank 28

- FY 91 -

$10 / 1 / 86$ start analysls of evaporator system $2 \mathrm{H}$

10/ 1/86 Begin to use recelving Tank 41

- $F Y 87$ -

$1 / 12 / 87$ Begin to use recelving Tank 38

- EY $8 \theta-$

- $8 Y 99-$

- EY 90 -

$5 / 21 / 90$ Begln to uge recelving Tank 11

- FY 91 -

Figure 9. Full listing of short $\log$ files for all four evaporator systems created from a four year simulation. 
The full log file used in Fig. 8 actually contains over eight pages of Information. In contrast, Fig, 9 shows the shorter log flles generated for all four of the evaporators over the same four year simulation. Much of the detailed information on how the simulation actually operated is lost; however, the most useful data such as when recelving tanks are switched and the number of inter-area transfers made is retained. Also note that a message indicating that evaporation had to be stopped untll salt removal was completed in tank 29 is written in the $1 \mathrm{H} \log$ file. Use of the shorter log files decreases the program execution time and provides all of the data that is normally required.

There are a few restrictions on the simulations that can be run with the WATFAM program. In writing the program, it was assumed that waste removal from type IV tanks would occur before sludge washing or DWPF recycle begin. The model attempts to complete waste removal before using sludge wash water or making inter-area transfers from $\mathrm{H}$-Area to $\mathrm{F}$ Area. This restriction may be avoided by including sludge wash water, waste removal, and DWPF recycle in the miscellaneous input volumes rather than as separate process streams. However, the miscellaneous volumes are input to the evaporator systems at a constant rate and the flexibility of allowing the model to process waste volume as space is available is lost with this approach. Since the waste removals were intended to be used in fiscal year 1986, they should no longer impact the simulation.

The model is also programmed to track fiscal years using only the last two digits of the year. This restricts process starting dates (Fig. A4) to dates within the same century. If dates in different centuries are entered, the program may not be able to order the process sequence correctly. For example, FY 1986 is 86 and FY 2001 is 01 in the program memory. Then, If DWPF recycle is started in 90.1 and the simulation begins on 01.1 , the program will not recognize that DWPF recycle is already in operation. Similarly, If DWPF recycle is to start in 01.1 and the simulation begins on 90.1, the program will think that the recycle is already operating. It is also assumed that all dates are later than 1980 in the READER program discussed below. If necessary, some additional programming could remove these minor restrictions on the model parameters.

\section{READER - Simulation Output Retrieval}

Output from an execution of the WATFAM simulation program is written to data flles TANK.DAT and EVAP.DAT in binary format. This data can be read and coples saved for printing by running the FORTRAN program READER. Output files generated using the data presented in the Appendix are used in this section to demonstrate the READER program and to show modol capabilities. The program begins by displaying a list of reading options to choose from. Fig. 10a shows the menu that appears on the terminal screen when READER is executed. The user can select one of five program options that generate output or the user can stop the program. Typical output displays that appear with each of the options are shown below.

The screen display when Option 1 is executed is shown in Fig. 10b. The user first selects the waste tank for which data will be read by entering a number between 1 and 51 . The user then selects how the output data is to be displayed. The example illustrates a request to write the data to an output file for later printing or further analysis. The user is prompted to enter a name for the file and one line of information that will appear at the start of the fili: for identification and documentation. The data may also be displayed on the terminal screen for immediate evaluation and either or both of these display options may be chosen. In the example, an output data file is created and the data is displayed on the terminal screen. A partial screen display appears at the bottom of Fig. $10 \mathrm{~b}$. 


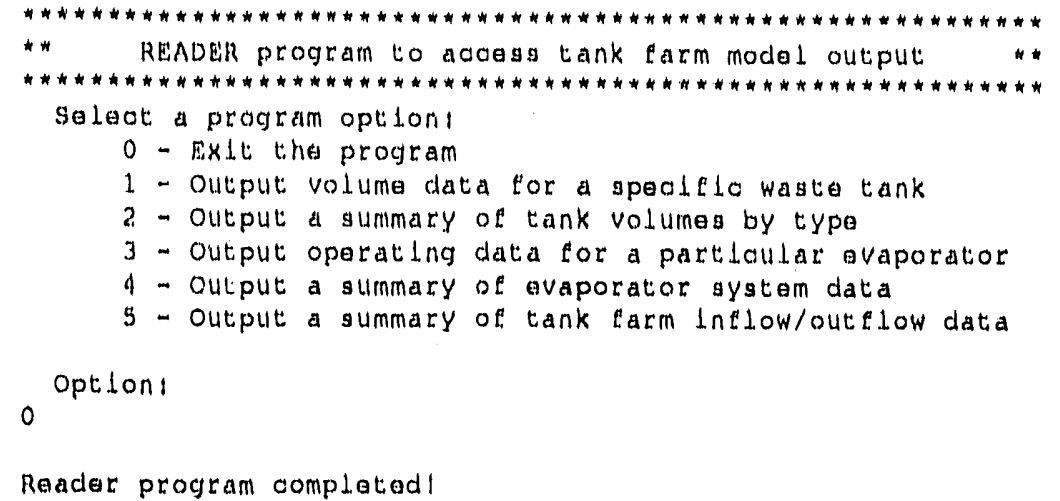

Figure 10a. Screen display of the menu from program READER.

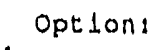

Data for tank number 43

\begin{tabular}{|c|c|c|c|c|c|c|c|}
\hline Data & $\begin{array}{l}\text { Total } \\
\text { (Kgal) }\end{array}$ & $\begin{array}{l}\text { valume } \\
\text { (In) }\end{array}$ & $\begin{array}{r}\text { Salt } \\
(\text { Kgal) }\end{array}$ & $\begin{array}{l}\text { Volume } \\
\qquad(1 n)\end{array}$ & $\begin{array}{l}\text { Sludge } \\
\text { (Kgal) }\end{array}$ & $\begin{array}{l}\text { Volume } \\
\text { (1n) }\end{array}$ & $\begin{array}{l}\text { Tank } \\
\text { SPG }\end{array}$ \\
\hline $10 / 1 / 86$ & 1221.8 & 340.1 & 0.0 & 0.0 & 117.9 & 33.6 & 1.340 \\
\hline $11 / 2 / 86$ & 1177.7 & 335.5 & 0.0 & 0.0 & 118.2 & 33.7 & 1.337 \\
\hline $12 / 1 / 86$ & 1122.6 & 319.8 & 0.0 & 0.0 & 118.5 & 33.8 & 1.330 \\
\hline $1 / 1 / 87$ & 1095.8 & 312,2 & 0.0 & 0.0 & 118.8 & 33,9 & 1.328 \\
\hline $2 / 1 / 87$ & 817.1 & 232,8 & 0.0 & 0.0 & 119.1 & 33,9 & 1.296 \\
\hline
\end{tabular}

Figure 10b. Screen display with Option 1 of program READER. 
Options 2 through 5 operate in essentially the same way allowing the user to save data in an output file or display results on the terminal screen. With Options 1,2 and 3, the data is output at the time intervals chosen when the WATFAM simulation program was run. For Options 4 and 5, the user can select monthly or yearly data summaries. Yearly data is reported on a fiscal year basis since that corresponds to a tank farm operating year. Actually, the time interval for data output is selected when WATFAM is run. If a datly or monthly calculation interval was used then options 4 and 5 in READER will give exact summaries. If some different time interval was selected, these options will give approximate summarles ending as close to the change in month or year as possible. That is, the program accumulates summary data untll a change in the month or year is detected at which point the summary is reported. If data was not saved at time intervals corresponding to an exact change in month or year, the summary will only be approximately correct.

A complete listing of the data saved in the TANK43 output file generated using Option 1 is shown in Fig. 11. The volumes of total waste, salt cake, and sludge are reported in kgal and in inches of tank level. The tank level assumes that the material in the tank is separated Into uniform layers with level surfaces. In actual practice, the salt cake and sludge accumulate in an irregular mannor and the predicted tank levels will correspond to average values that only approximately agree with measured levels. The specific gravity of the tank liquid waste is also given. Data values are reported at the start of the indicated date.

Tank 43 is the feed tank for the $2 \mathrm{H}$ evaporator. As Fig. 11 shows, the volume in the tank fluctuates considerably from month to month as material is fed to the evaporator, fresh waste enters the tank, and concentrate is recycled. As the waste composition changes, the liquid specific gravity also changes. Sludge is assumed to separate from fresh waste in the feed tank and accumulates at a relatively constant rate.

Data from the corresponding simulation for tank 38 are plotted in Fig. 12. Near the start of FY 1987, tank 38 becomes the recelving tank for the $2 \mathrm{H}$ evaporator. The total volume of waste in the tank increases as concentrate from the evaporator is added to the tank and decreases when supernatant liquid is recycled. As explained above, tank 38 will contain a large volume of low specific gravity waste when it is first used. Therefore, salt cake does not start to precipitate out until about four months have elapsed. The volume of salt cake in the tank then increases steadily until $1000 \mathrm{kgal}$ have accumulated at about the midpoint of FY 1989. At this point, recycle is stopped and the tank is filled to the operating limit with concentrate. Since the model waits until $1000 \mathrm{kgal}$ of salt cake are already present to top off the tank, a small amount of additional salt cake precipitates out during this process. In this simulation, tank 38 is completely full at approximately the start of FY 1990 when tank 41 must again be used as the concentrate receiving tank. Actual tank farm operations during the indicated time period did not correspond to the assumptions used for this example simulation and tank 38 is still not full of salt cake as of early 1991. 
Tank farm slmulation tast: data for tank 43

Data for tank nunber 43

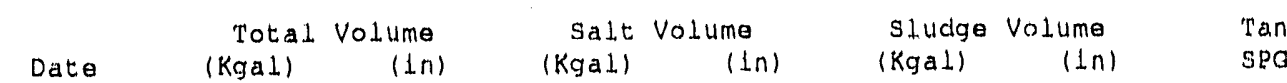

\begin{tabular}{|c|c|c|c|c|c|c|c|c|}
\hline 101 & $1 / 86$ & 1221.8 & 348.1 & .0 & .0 & 117.9 & 33,6 & 1.340 \\
\hline $11 /$ & $1 / 86$ & 1177.7 & 335.5 & .0 & .0 & 118.2 & 33.7 & 1.337 \\
\hline 121 & $1 / 86$ & 1122.6 & 319.8 & .0 & .0 & 118.5 & 33.8 & 1,330 \\
\hline $1 /$ & $1 / 87$ & 1095.8 & 312.2 & .0 & .0 & 118.8 & 33.9 & 1.328 \\
\hline 21 & $1 / 87$ & 817.1 & 232.8 & .0 & .0 & 119.1 & 33,9 & 1.296 \\
\hline $3 /$ & $1 / 87$ & 551.0 & 157.0 & .0 & .0 & 119.4 & 34.0 & 1.242 \\
\hline 41 & $1 / 87$ & 916.5 & 261.1 & .0 & .0 & 119.7 & 34.1 & 1.293 \\
\hline $5 /$ & $1 / 07$ & 633.2 & 180.4 & .0 & .0 & 120.0 & 34.2 & 1.249 \\
\hline $6 /$ & $1 / 87$ & 370.5 & 105.6 & .0 & .0 & 120.3 & 34.3 & 1.196 \\
\hline 71 & $1 / 87$ & 378.4 & 107.8 & .0 & .0 & 120.6 & 34.4 & 1.250 \\
\hline $8 /$ & $1 / 87$ & 1176.4 & 335,2 & .0 & .0 & 120.9 & 34.5 & 1.389 \\
\hline 9/ & $1 / 87$ & 651.0 & 185.5 & .0 & .0 & 121.2 & 34.5 & 1,332 \\
\hline $10 /$ & $1 / 87$ & 744.4 & 212.1 & .0 & .0 & 121.5 & 34.6 & 1.362 \\
\hline $11 /$ & $1 / 87$ & 510.8 & 145.5 & .0 & .0 & 121.9 & 34.7 & 1.335 \\
\hline 121 & $1 / 87$ & 466.9 & 133.0 & .0 & .0 & 122.3 & 34.9 & 1.359 \\
\hline $1 /$ & $1 / 88$ & 421.4 & 120.1 & .0 & .0 & 122.7 & 35.0 & 1.348 \\
\hline 21 & $1 / 88$ & 384.1 & 109.4 & .0 & .0 & 12.3 .1 & 35.1 & 1.340 \\
\hline $3 /$ & $1 / 88$ & 376.6 & 107.3 & .0 & .0 & 123.5 & 35.2 & 1.334 \\
\hline $4 /$ & $1 / 88$ & 598.6 & 170.6 & .0 & .0 & 123,9 & 35.3 & 1,384 \\
\hline $5 /$ & $1 / 80$ & 403.9 & 115.1 & .0 & .0 & 1.24 .3 & 35.4 & 1. . 356 \\
\hline $6 /$ & $1 / 88$ & 408.1 & 116.3 & .0 & .0 & 124.7 & 35.5 & 1.315 \\
\hline 71 & $1 / 88$ & 367.0 & 104.6 & .0 & .0 & 125.1 & 35.6 & 1.320 \\
\hline $8 /$ & $1 / 88$ & 447.8 & 127.6 & .0 & .0 & 125.5 & 35.8 & 1.257 \\
\hline $9 /$ & 1.180 & 399.5 & 113.8 & .0 & .0 & 125.9 & 35.9 & 1.269 \\
\hline $10 /$ & $1 / 88$ & 385.7 & 109.9 & .0 & .0 & 126.3 & 36.0 & 1.273 \\
\hline $11 /$ & $1 / 88$ & 399.8 & 113.9 & .0 & .0 & 126,9 & 36.1 & 1.203 \\
\hline 121 & $1 / 88$ & 363.7 & 103.6 & .0 & .0 & 127.4 & 36.3 & 1.128 \\
\hline $1 /$ & $1 / 89$ & 367.9 & 104.8 & .0 & .0 & 127.9 & 36.4 & 1.113 \\
\hline 21 & $1 / 89$ & 387.8 & 110.5 & .0 & .0 & 128.4 & 36.6 & 1.119 \\
\hline $3 /$ & $1 / 89$ & 427.3 & 121.7 & .0 & .0 & 128.9 & 36.7 & 1.160 \\
\hline $4 /$ & $1 / 89$ & 363.2 & 103.5 & .0 & .0 & 129.4 & 36.9 & 1.093 \\
\hline $5 /$ & $1 / 89$ & 422.3 & 120.3 & .0 & .0 & 129.9 & 37.0 & 1.131 \\
\hline $6 /$ & $1 / 89$ & 368.6 & 105.0 & .0 & .0 & $1,30.5$ & 37.2 & 1.083 \\
\hline 71 & $1 / 89$ & 499.0 & 142.2 & .0 & .0 & 131.0 & 37.3 & 1.200 \\
\hline $8 /$ & $1 / 89$ & 415.6 & 118.4 & .0 & .0 & 131.5 & 37.5 & 1.120 \\
\hline $9 /$ & $1 / 89$ & 467.4 & 133.2 & .0 & .0 & 132.1 & 37.6 & 1.176 \\
\hline $10 /$ & $1 / 89$ & 400,2 & 114.0 & .0 & .0 & 132.6 & 37.8 & 1.106 \\
\hline $11 /$ & $1 / 89$ & 521.2 & 148.5 & .0 & .0 & 1.33 .0 & 37.9 & 1.269 \\
\hline 121 & $1 / 89$ & 397.9 & 113.4 & .0 & .0 & 133.3 & 38.0 & 1.217 \\
\hline $1 /$ & $1 / 90$ & 428.1 & 122.1 & .0 & .0 & 133.7 & 38.1 & 1.121 \\
\hline 21 & $1 / 90$ & 437,9 & 124.8 & .0 & .0 & 1.34 .1 & 38.2 & 1.077 \\
\hline $3 /$ & $1 / 90$ & 363.0 & 103.4 & .0 & .0 & 134.5 & 38.3 & 1.059 \\
\hline 41 & $1 / 90$ & 387.6 & 110.1 & .0 & .0 & 134.9 & 38,4 & 1.049 \\
\hline $5 /$ & $1 / 90$ & 419.9 & $.219,6$ & .0 & .0 & 135.2 & 38.5 & 1.045 \\
\hline $6 /$ & $1 / 90$ & 396.2 & 112.9 & .0 & .0 & 135.6 & 38.6 & 1.042 \\
\hline 71 & $1 / 90$ & 367.4 & 104.7 & .0 & .0 & 136.0 & 38.7 & 1.042 \\
\hline $8 /$ & $1 / 90$ & 431.7 & 123.0 & .0 & .0 & 136.4 & 38.9 & 1.041 \\
\hline $9 /$ & $1 / 90$ & 396.5 & 113.0 & .0 & .0 & 136,8 & 39.0 & 1.040 \\
\hline 101 & $1 / 90$ & 343.1 & 97.7 & .0 & .0 & 137.2 & 39.1 & 1.041 \\
\hline
\end{tabular}

Figure 11. Listing of model output for tank 43 from a four year simulation. 


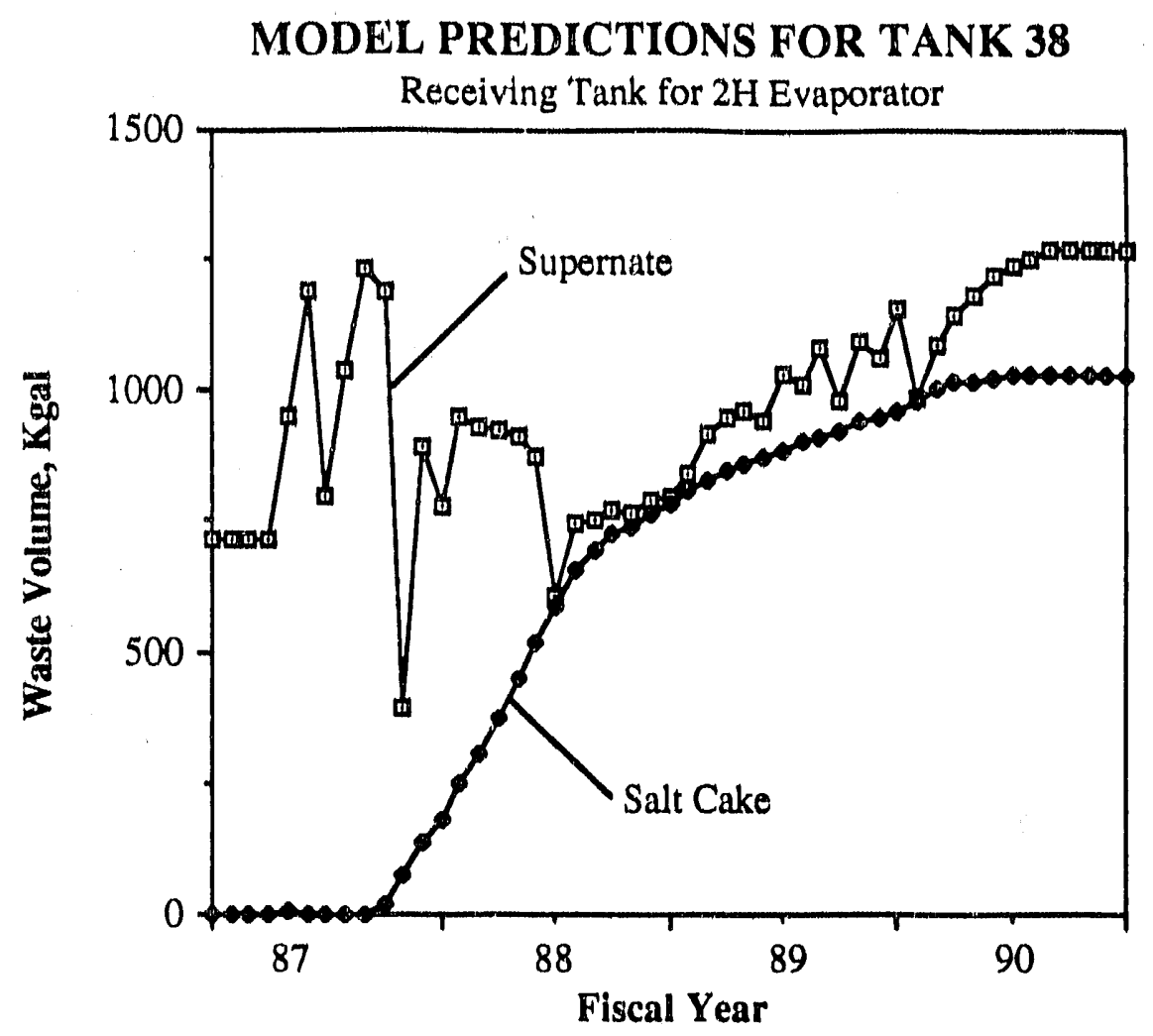

Figure 12. Model predictions of waste volumes in tank 38.

Option 2 outputs volume data for all waste tanks of a particular type (I, II, III, or IV), for the four fresh waste receiving tanks (tanks 33,34,35, and 39) in the high heat system, or for all waste tanks in the tank farm. We note that since the code was written, tank 32 is now also being used as a fresh waste receiving tank in the H-Area high heat waste system. At this time, the coding has not been modified to include tank 32 in the output summary of high heat waste receivers and its contents must be included manually if so desired. Data for type III waste tanks from the four year example simulation are shown in Fig. 13. The volumes of total waste, salt cake, and sludge are given in $\mathrm{kgal}$ and in inches of tank level. Simulation results for the waste volume in all of the waste tanks are plotted in Fig. 14. The simulation predicts a slow decrease in the total waste volume and a slight increase in sludge volume over the four years. Salt cake builds up until salt removal begins in FY 1988 when a gradual decline in volume starts. 
Four year simulation data for Type III waste tanks:

Data for type III tanks

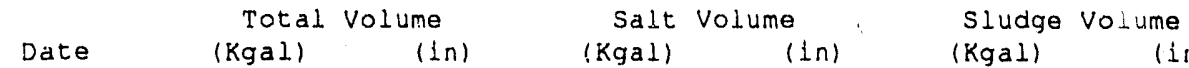

\begin{tabular}{|c|c|c|c|c|c|c|c|}
\hline 101 & $1 / 86$ & 25049.1 & 7136.5 & 8260.4 & 2353.4 & 1206.0 & 343.6 \\
\hline $11 /$ & $1 / 86$ & 24852.0 & 7080.3 & 8441.3 & 2404.9 & 1211.0 & 345.0 \\
\hline 121 & $1 / 86$ & 24667.2 & 7027.7 & 8680.7 & 2473.1 & 1216.0 & 346.4 \\
\hline $1 /$ & $1 / 87$ & 24507.9 & 6982.3 & 8973.9 & 2556.7 & 1220.9 & 347.8 \\
\hline $2 /$ & $1 / 87$ & 24478.1 & 6973.8 & 9167.7 & 2611.9 & 1225.9 & 349.3 \\
\hline $3 /$ & $1 / 87$ & 24436.2 & 6961.9 & 9301.2 & 2649.9 & 1230.9 & 350.7 \\
\hline $4 /$ & $1 / 87$ & $2<952.7$ & 7109.0 & 9515.7 & 2711.0 & 1651.8 & 470.6 \\
\hline $5 /$ & $1 / 87$ & 24855.1 & 7081.2 & 9641.1 & 2746.8 & 1656.7 & 472.0 \\
\hline $6 /$ & $1 / 87$ & 24695.5 & 7035.8 & 9690.1 & 2760.7 & 1661.7 & 473.4 \\
\hline $7 /$ & $1 / 87$ & 24622.4 & 7014.9 & 9808.0 & 2794.3 & 1666.7 & 474.8 \\
\hline $8 /$ & $1 / 87$ & 24622.6 & 7015.0 & 9941.1 & 2832.2 & 1671.7 & 476.3 \\
\hline $9 /$ & $1 / 87$ & 24613.2 & 7012.3 & 10099.5 & 2877.3 & 1676.6 & 477.7 \\
\hline $10 /$ & $1 / 87$ & 24586.6 & 7004.7 & 10242.9 & 2918.2 & 1681.6 & 479.1 \\
\hline $11 /$ & $1 / 87$ & 24516.2 & 6984.7 & 10392.4 & 2960.8 & 1689.4 & 481.3 \\
\hline $12 /$ & $1 / 87$ & 24447.2 & 6965.0 & 10549.2 & 3005.5 & 1696.9 & 483.5 \\
\hline $1 /$ & $1 / 88$ & 24369.3 & 6942.8 & 10750.6 & 3062.9 & 1704.7 & 485.7 \\
\hline 21 & $1 / 88$ & 24274.5 & 6915.8 & 10964.8 & 3123.9 & 1712.5 & 487.9 \\
\hline $3 /$ & $1 / 88$ & 24244.5 & 6907.3 & 11132.7 & 3171.7 & 1719.5 & 489.9 \\
\hline $4 /$ & $1 / 88$ & 24215.6 & 6899.0 & 11322.7 & 3225.8 & 172.7 .3 & 492.1 \\
\hline $5 /$ & $1 / 88$ & 24174.7 & 6887.4 & 11434.5 & 3257.7 & 1734.9 & 494.3 \\
\hline $6 /$ & $1 / 88$ & 24150.5 & 6880.5 & 11540.6 & 3287.9 & 1742.7 & 496.5 \\
\hline $7 /$ & $1 / 88$ & 24153.2 & 6881.2 & 11593.5 & 3303.0 & 1750.2 & 498.6 \\
\hline 81 & $1 / 88$ & 24309.0 & 6925.6 & 11612.5 & 3308.4 & 1758.0 & 500.8 \\
\hline $9 /$ & $1 / 88$ & 24073.6 & 6858.6 & 11215.1 & 3195.2 & 1899.3 & 541.1 \\
\hline $10 /$ & $1 / 88$ & 24026.7 & 6845.2 & 11374.8 & 3240.7 & 1906.8 & 543.3 \\
\hline $11 /$ & $1 / 88$ & 24039.7 & 6848.9 & 11585.5 & 3300.7 & 1916.7 & 546.1 \\
\hline $12 /$ & $1 / 88$ & 24145.0 & 6878.9 & 11707.2 & 3335.4 & 1926.2 & 548.8 \\
\hline $1 /$ & $1 / 89$ & 24068.1 & 6857.0 & 11312.2 & 3222.8 & 2069.5 & 589.6 \\
\hline 21 & $1 / 89$ & 24182.0 & 6889.4 & 11421.1 & 3253.9 & 2079.4 & 592.4 \\
\hline 31 & $1 / 89$ & 24260.4 & 6911.8 & 11490.7 & 3273.7 & 2080.3 & 595.0 \\
\hline $4 /$ & $1 / 89$ & 24453.5 & 6966.8 & 11574.1 & 3297.5 & 2586.2 & 736.8 \\
\hline $5 /$ & $1 / 89$ & 24118.1 & 6871.2 & 11181.2 & 3185.5 & 2729.2 & 777.6 \\
\hline $6 /$ & $1 / 89$ & 24192.9 & 6892.6 & 11266.4 & 3209.8 & 2739.1 & 780.4 \\
\hline $7 /$ & $1 / 89$ & 24308.2 & 6925.4 & 11354.4 & 3234.9 & 2748.6 & 783.1 \\
\hline $8 /$ & $1 / 89$ & 24394.8 & 6950.1 & 11458.2 & 3264.4 & 2758.5 & 785.9 \\
\hline $9 /$ & $1 / 89$ & 24116.3 & 6870.8 & 11060.3 & 3151.1 & 2902.1 & 826.8 \\
\hline $10 /$ & $1 / 89$ & 24276.0 & 6916.2 & 11141.3 & 3174.2 & 2911.6 & 829.5 \\
\hline $11 /$ & $1 / 89$ & 24363.3 & 6941.1 & 11215.7 & 3195.4 & 2918.8 & 831.6 \\
\hline $12 /$ & $1 / 89$ & 24409.6 & 6954.3 & 11288.6 & 3216.1 & 2925.8 & 833.6 \\
\hline $1 /$ & $1 / 90$ & 24198.3 & 68.94 .1 & 10993.8 & 3132.1 & 3053.5 & 869.9 \\
\hline 21 & $1 / 90$ & 24267.4 & 6913.8 & 11057.3 & 3150.2 & 3060.7 & 872.0 \\
\hline $3 /$ & $1 / 90$ & 24377.4 & 6945.1 & 11111.9 & 3165.8 & 3067.2 & 873.8 \\
\hline $4 /$ & $1 / 90$ & 24467.0 & 6970.6 & 11174.2 & 3183.5 & 3074.4 & 875.9 \\
\hline $5 /$ & $1 / 90$ & 21364.8 & 6941.5 & 10855.5 & 3092.7 & 3208.3 & 914.0 \\
\hline $6 /$ & $1 / 90$ & 24481.7 & 6974.9 & 10915.2 & 3109.7 & 3215.5 & 916.1 \\
\hline $7 /$ & $1 / 90$ & 23062.8 & 6570.6 & 10979.2 & 3128.0 & 3198.3 & 911.2 \\
\hline $8 /$ & $1 / 90$ & 23240.6 & 6621.3 & 11008.8 & 3136.4 & 3181.3 & 906.4 \\
\hline $9 /$ & $1 / 90$ & 23121.4 & 6587.3 & 10595.6 & 3018.7 & 3291.2 & 937.7 \\
\hline $10 /$ & $1 / 90$ & 23108.4 & 6583.6 & 10642.6 & 3032.1 & 3274.0 & 932.8 \\
\hline
\end{tabular}

Figure 13. Model simulation output for Type III waste tanks. 


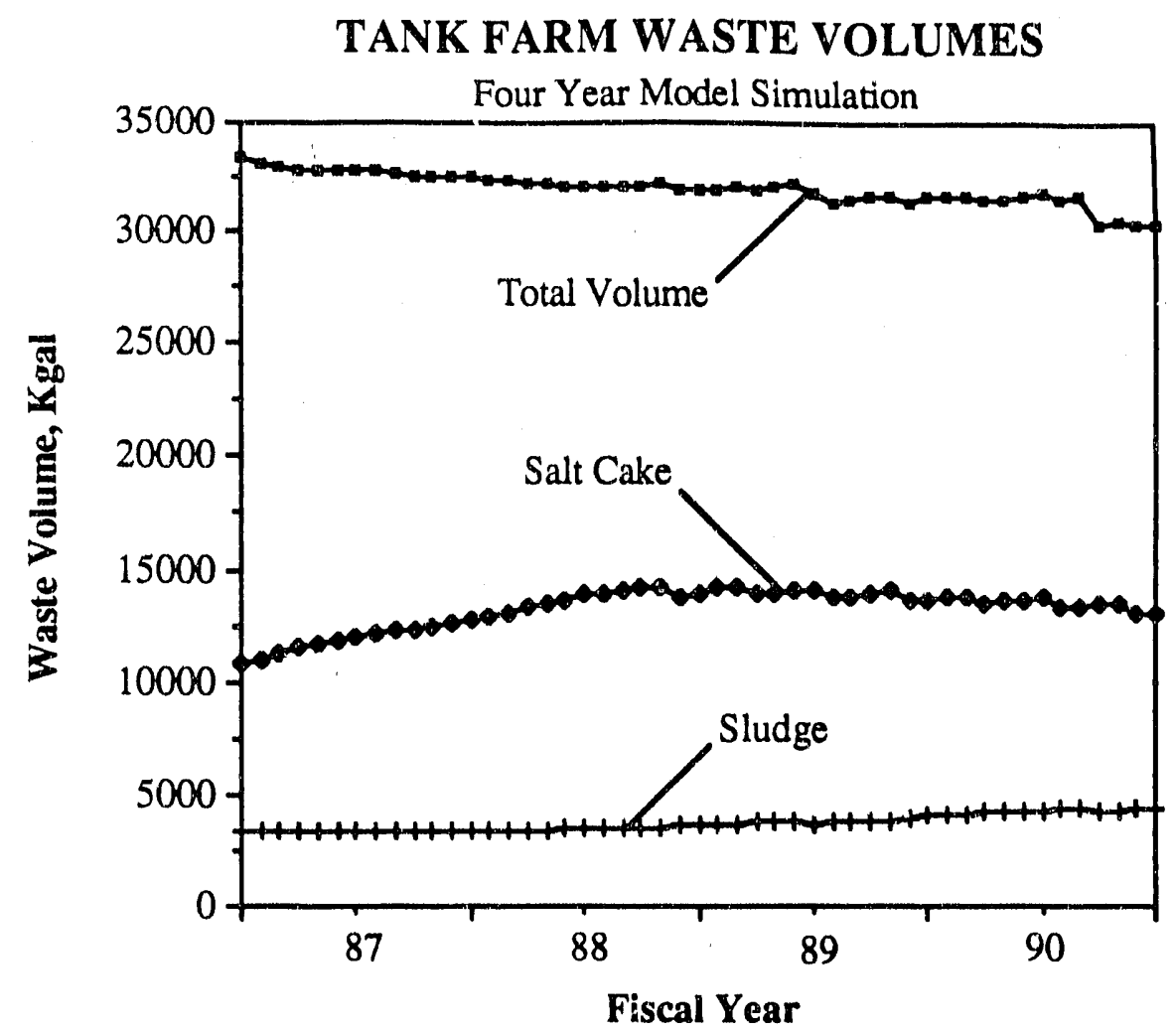

Figure 14. Model predictions of waste volumes within all waste tanks over a four year interval.

A listing of the output data from Option 3 for the $2 \mathrm{H}$ evaporator is presented in Fig. 15. The data reports average flow rates for the overhead, concentrate, and feed streams. Average flow rates for the concentrate and feed streams are calculated by summing the integrated flows from Eqs. (A20) and (A21) over the time period between printouts and dividing by the number of days that the evaporator operated during that time. The net overhead rate is equal to the difference between the feed and concentrate streams. The steam lance is not included in the net overhead rate. A plot of the average flow rates in Fig. 16 shows that the evaporator overheads remain fairly constant despite large changes in the other streams. The feed and concentrate flows increase when tank 38 is placed in service as the concentrate receiving tank and decline as the tank fills.

The feed specific gravity is the specific gravity of the evaporator feed stream at the start of the listed day. For the $2 \mathrm{H}$ evaporator, this is the same as the specific gravity of liquid waste in feed tank 43. Net space gain is calculated as net overheads less flush water and recycle dilution to give the increase in tank farm capacity from evaporator operation. We assume that any lance dilution is immediately removed in the evaporators and so makes no contribution to computation of net space gain. 
Four year simulation output:

Data for evaporator system $2 \mathrm{H}$ Average Averacje Overhead Concentrat Date ( KgaL/Day) (Kgal/ Feed Feed

Net

Space Galn Percent (Kgal) Operation

\begin{tabular}{|c|c|c|c|c|c|c|c|}
\hline 101 & $1 / 86$ & .00 & .00 & .00 & 1.340 & .00 & .00 \\
\hline $11 /$ & $1 / 86$ & 9.31 & 14.96 & 24.27 & 1.337 & 184.13 & 77.42 \\
\hline 121 & $1 / 86$ & 9.31 & 14.93 & 24.24 & 1.330 & 177.34 & 76.67 \\
\hline $1 /$ & $1 / 87$ & 9.32. & 14.83 & 24.15 & 1.328 & 184.00 & 77.42 \\
\hline 21 & $1 / 87$ & 9.43 & 13.43 & 22.86 & 1.296 & 199.81 & 77.42 \\
\hline $3 /$ & $1 / 87$ & 9.76 & 9.99 & 19.75 & 1.242 & 199.34 & 78.57 \\
\hline $4 /$ & $1 / 87$ & 9.79 & 10.08 & 19.87 & 1.293 & 192.12 & 77.42 \\
\hline $5 /$ & $1 / 87$ & 9.77 & 9.86 & 19.64 & 1.249 & 208.92 & 76.67 \\
\hline $6 /$ & $1 / 87$ & 10.14 & 7.10 & 17.24 & 1.196 & 241.20 & 80.65 \\
\hline $7 /$ & $1 / 87$ & 9.68 & 11.05 & 20.73 & 1.250 & 200.87 & 80.00 \\
\hline $8 /$ & $1 / 87$ & 9.43 & 14.47 & 23.90 & 1.389 & 163.25 & 80.65 \\
\hline $9 /$ & $1 / 87$ & 9.08 & 18.66 & 27.75 & 1.332 & 194.45 & 80.65 \\
\hline $10 /$ & $1 / 87$ & 9.56 & 12.29 & 21.84 & 1.362 & 191.48 & 80.00 \\
\hline $1.1 /$ & $1 / 87$ & 9.09 & 18.47 & 27.57 & 1.335 & 182.06 & 80.65 \\
\hline $12 /$ & $1 / 87$ & 9.25 & 16.11 & 25.36 & 1.359 & 177.71 & 80.00 \\
\hline $1 /$ & $1 / 88$ & 9.02 & 20.01 & 29.03 & 1.348 & 168.99 & 80.65 \\
\hline 21 & $1 / 88$ & 9.04 & 19.66 & 28.70 & 1.340 & 170.04 & 80.65 \\
\hline $3 /$ & $1 / 88$ & 9.01 & 20.27 & 29.29 & 1.334 & 153.28 & 82.14 \\
\hline $4 /$ & $1 / 88$ & 9.08 & 19.10 & 28.18 & 1.384 & 162.48 & 80.65 \\
\hline $5 /$ & $1 / 88$ & 9.03 & 19.62 & 28.66 & 1.356 & 169.33 & 80.00 \\
\hline $6 /$ & $1 / 88$ & 9.09 & 18.60 & 27.69 & 1.315 & 111.98 & 51.61 \\
\hline 71 & $1 / 88$ & 9.52 & 12.61 & 22.13 & 1.320 & 138.38 & 56.67 \\
\hline 81 & $1 / 88$ & 9.34 & 14.70 & 24.04 & 1.257 & 38.41 & 16.13 \\
\hline $9 /$ & $1 / 88$ & 9.79 & 9.97 & 19.76 & 1.269 & 148.04 & 54.84 \\
\hline $10 /$ & $1 / 88$ & 9.95 & 8.59 & 18.55 & 1.273 & 117.29 & 43.33 \\
\hline $11 /$ & $1 / 88$ & 10.03 & 7.94 & 17.97 & 1.203 & 230.03 & 80.65 \\
\hline $12 /$ & $1 / 88$ & 10.48 & 5.20 & 15.68 & 1.128 & 240.38 & 80.00 \\
\hline $1 /$ & $1 / 89$ & 10.65 & 4.39 & 15.04 & 1.113 & 254.94 & 80.65 \\
\hline 21 & $1 / 89$ & 10.65 & 4.39 & 15.04 & 1.119 & 254.52 & 80.65 \\
\hline $3 /$ & $1 / 89$ & 10.81 & 3.74 & 14.55 & 1.160 & 238.29 & 82.14 \\
\hline $4 /$ & $1 / 89$ & 10.91 & 3.16 & 14.06 & 1.093 & 267.17 & 80.65 \\
\hline $5 /$ & $1 / 89$ & 10.53 & 5.02 & 15.55 & 1.131 & 238.43 & 80.00 \\
\hline $6 /$ & $1 / 89$ & 11.04 & 2.60 & 13.65 & 1.083 & 271.57 & 80.65 \\
\hline 71 & $1 / 89$ & 10.87 & 3.75 & 14.62 & 1.200 & 246.75 & 80.00 \\
\hline $8 /$ & $1 / 89$ & 10.68 & 4.15 & 14.84 & 1.120 & 259.70 & 80.65 \\
\hline $9 /$ & $1 / 89$ & 11.00 & 2.92 & 13.92 & 1.176 & 265.48 & 80.65 \\
\hline $10 /$ & $1 / 89$ & 10.80 & 3.63 & 14.42 & 1.106 & 253.01 & 80.00 \\
\hline $11 /$ & $1 / 89$ & 10.71 & 4.32 & 15.04 & 1.269 & 248.91 & 80.65 \\
\hline $12 /$ & $1 / 89$ & 10.03 & 7.94 & 17.97 & 1.217 & 223.00 & 80.00 \\
\hline $1 /$ & $1 / 90$ & 10.51 & 5.03 & 15.54 & 1.121 & 111.70 & 35.48 \\
\hline 21 & $1 / 90$ & 11.02 & 2.69 & 13.71 & 1.077 & 1.51 .67 & 45.16 \\
\hline $3 /$ & $1 / 90$ & 11.30 & 1.65 & 12.95 & 1.059 & 223.78 & 71.43 \\
\hline $4 /$ & $1 / 90$ & 11.44 & 1.20 & 12.64 & 1.049 & 158.98 & 45.16 \\
\hline $5 /$ & $1 / 90$ & 11.47 & 1.10 & 12.57 & 1.045 & 148.12 & 43.33 \\
\hline $6 /$ & $1 / 90$ & 11.50 & 1.01 & 12.51 & 1.042 & 205.65 & 58.06 \\
\hline $7 /$ & $1 / 90$ & 11.51 & .96 & 12.48 & 1.042 & 205.99 & 60.00 \\
\hline $8 /$ & $1 / 90$ & 11.52 & .95 & 12.47 & 1.041 & 125.94 & 35.48 \\
\hline $9 /$ & $1 / 90$ & 11.52 & .94 & 12.46 & 1.040 & 217.63 & 61.29 \\
\hline $10 /$ & $1 / 90$ & 11.52 & .94 & 12.46 & 1.041 & 229.09 & 66.67 \\
\hline
\end{tabular}

Figure 15. Model simulation output for the $2 \mathrm{H}$ evaporator. 




Figure 16. Model simulation of $2 \mathrm{H}$ evaporator operation over a four year period.

Percent operation is determined by dividing the total number of days in the reporting time period by the number of days that the evaporator operated. Days when the evaporator does not operate are randomly assigned based on the input utility. As the data in Fig. A9 shows, for the $2 \mathrm{H}$ evaporator, from $10 / 86$ to $5 / 87$ a utility of 0.77 was input, from $5 / 87$ through the rest of FY 1987 the utility was 0.8 , and 0.81 after FY 1987. Since the simulation operates on a daily basis, the utility numbers are not reproduced exactly. For example, no distinction can be made between the utilities of 0.8 and 0.81 with daily time increments. Nevertheless, specified utilities are reproduced with good accuracy. Figure 17 presents a plot of the space gain and percent operation data. Evaporator operation declines in late FY 1988 and again in FY 1990 as feed volume is depleted when the concentrate receiving tanks are filled and recycle is stopped.

Except for feed specific gravity, all of the data in Figs. 15-17 give values up to the listed dates. That is, the data for $11 / 1 / 86$ gives values accumulated between 10/1/86 and 11/1/86. The net space gain and percent operation are then the values for October 1986 and the average flow rates given on $11 / 1 / 86$ are calculated from operating data during October 1986. 


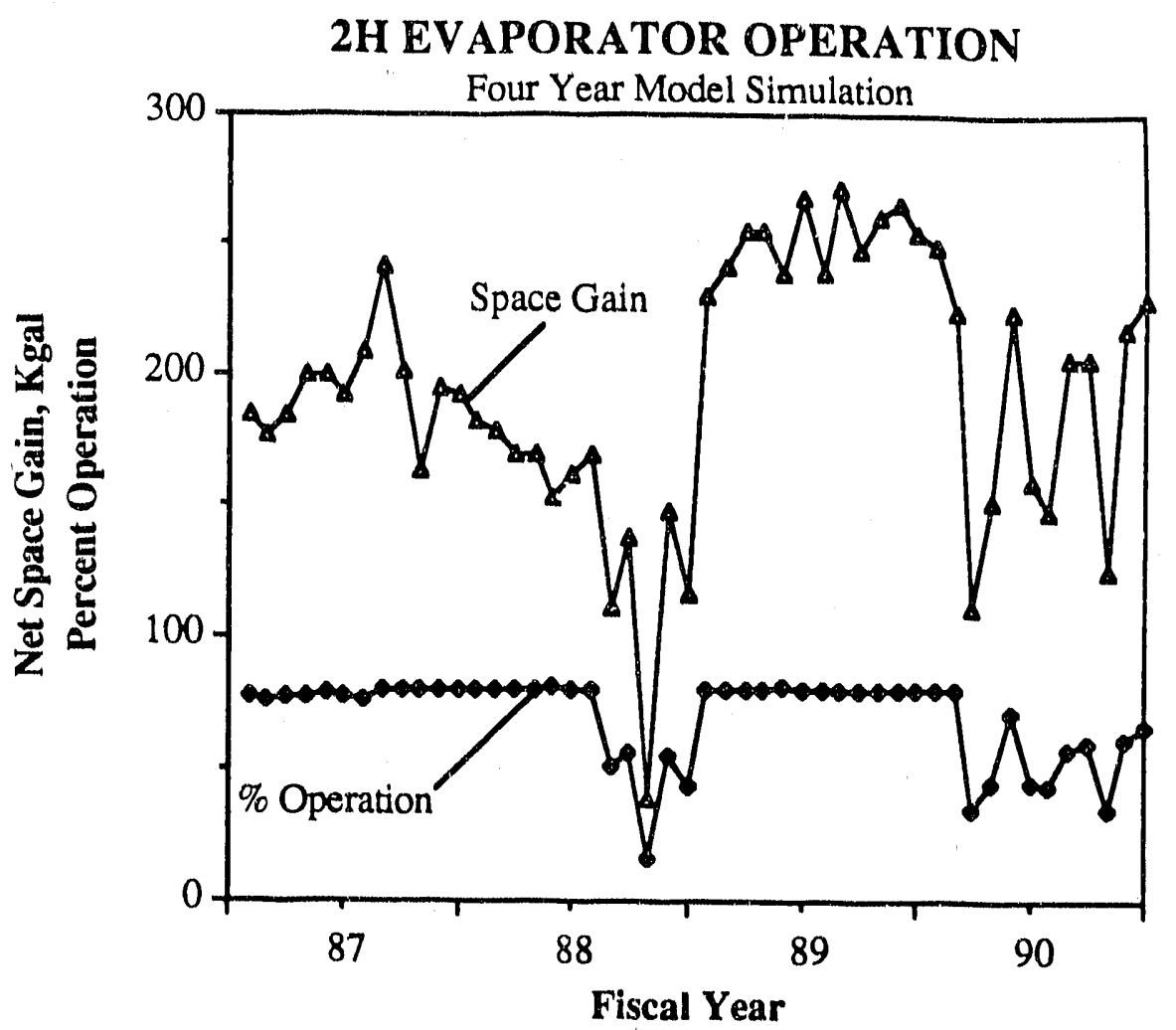

Figure 17. Model simulation of $2 \mathrm{H}$ evaporator net space gain and utility over a four year period.

A complete listing of output data from Option 4 for the $2 \mathrm{H}$ evaporator system is shown in Fig. 18. Four years were simulated with a starting date of $10 / 86$. Monthly or yearly summaries could be selected; since monthly data was saved when WATFAM was run, the yearly summaries are exact. The first line of data gives conditions at the start of the simulation. For summary purposes, three evaporator systems are reported. The $1 \mathrm{~F}$ and $2 \mathrm{~F}$ evaporators handle low heat waste from F-Area through feed tank 26 and are treated as a single system. The $1 \mathrm{H}$ cvaporator is the high heat waste evaporator for both separation areas and the $2 \mathrm{H}$ evaporator handles low heat waste in H-Area. Summary data for the $1 \mathrm{~F}$ $2 F$ system includes tanks $26,44,45,47,25,27$, and 28 . Data for the $1 \mathrm{H}$ system uses tanks $30,13,32$, and receiving tanks $29,31,36$, and 37 . The fresh waste receipt tanks in the high heat system are not included in the summary. We note that tank 30 will in fact be used as a salt receiving tank within the $1 \mathrm{H}$ system in the future and that tank 32 is not really a part of the $1 \mathrm{H}$ system until 1994 when it will replace tank 13 . When the new $1 \mathrm{H}$ evaporator is placed in service and tank 13 is removed from the $1 \mathrm{H}$ system, tank 13 is no longer included in the data report. This change will produce a slight discontinuity in the volume data. Some modifications to the existing programs are required to eliminate tank 32 from the $1 \mathrm{H}$ system summary until it replaces tank 13 (tank 32 is actually operating as a receiving tank for fresh high heat waste in H-Area) and to more correctly model the function of tank 30 . The $2 \mathrm{H}$ system data summary simply includes tanks 43,41 , and 38 .

The summary data gives the total volume of all waste, salt cake, and sludge in the evaporator system obtained by summing the individual contributions from each of the waste tanks listed above. The volume of liquid waste may be calculated by subtracting the 
salt cake and sludge volumes from the total. Figure 19 shows a plot of monthly volume data for the $2 \mathrm{H}$ evaporator system. Salt removals from the system are clearly indicated by immediate drops in the salt cake and total volumes.

Four yoar simulation output:

Conditions at start of date for $2 \mathrm{H}$ Evaporator:

\begin{tabular}{ccccrrr} 
Fotal & $\begin{array}{c}\text { Total } \\
\text { Volume } \\
\text { (Kgal) }\end{array}$ & $\begin{array}{c}\text { Salt } \\
\text { (Kgal) }\end{array}$ & $\begin{array}{c}\text { Total } \\
\text { (Kgal) }\end{array}$ & $\begin{array}{c}\text { Avallable Avallable Ner Space } \\
\text { (Kolume } \\
\text { (Kgal) }\end{array}$ & $\begin{array}{c}\text { Salt } \\
\text { (Kgal) }\end{array}$ & $\begin{array}{c}\text { Galn } \\
\text { (KgaL) }\end{array}$ \\
\hline 1987 & 3096.2 & 800.3 & 117.9 & 673.6 & 1199.7 & .0 \\
1988 & 2779.8 & 1189.2 & 121.5 & 990.0 & 816.4 & 2336.9 \\
1989 & 2044.0 & 1387.3 & 126.3 & 1313.6 & 218.3 & 1738.0 \\
1990 & 1913.8 & 1068.4 & 132.6 & 943.8 & 37.3 & 3020.3 \\
1991 & 1745.3 & 1040.8 & 137.2 & 2024.4 & 990.7 & 2250.4
\end{tabular}

Figure 18. Complete listing of yearly summary data for the $2 \mathrm{H}$ evaporator system from a four year simulation.

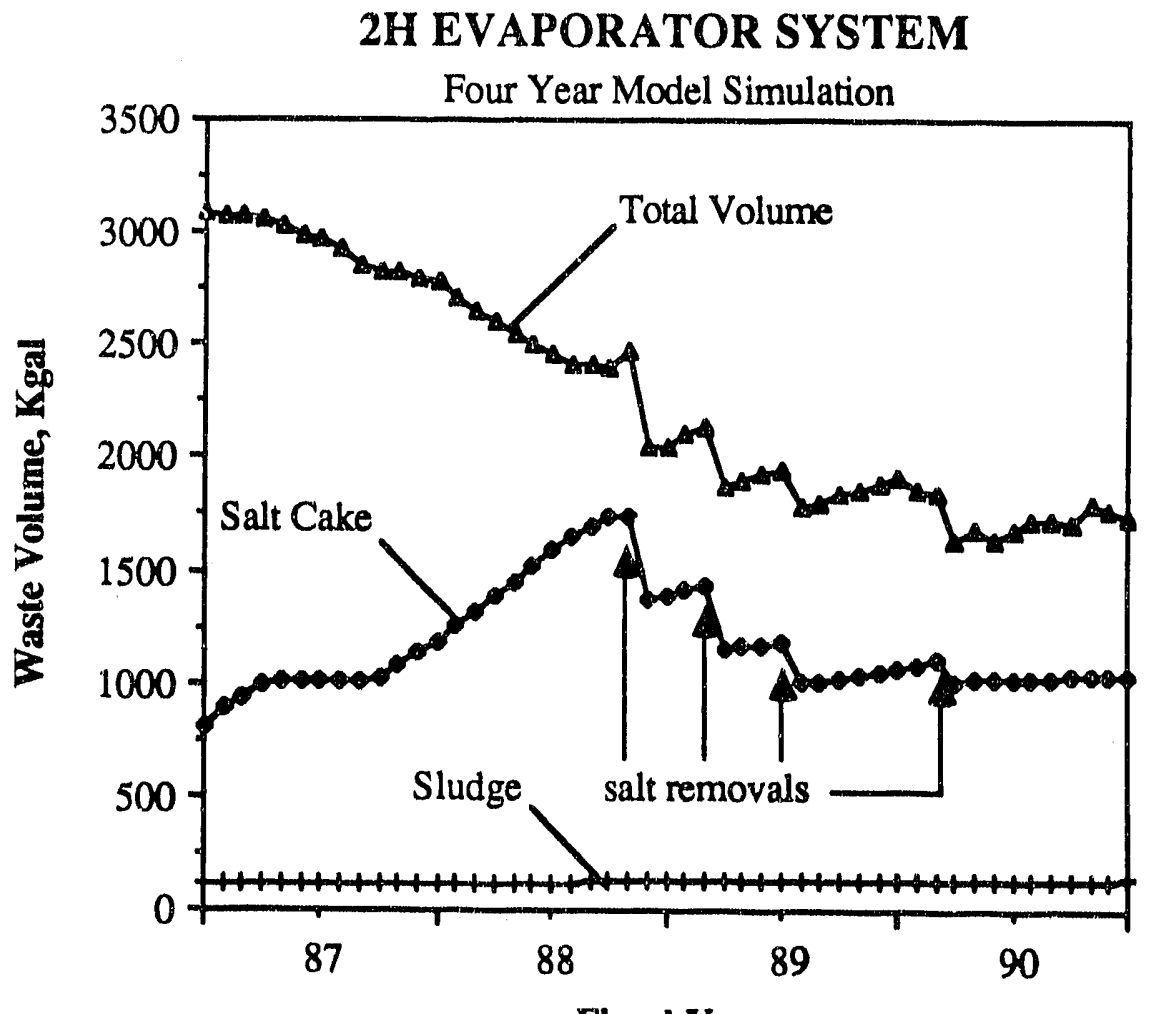

Fiscal Year

Figure 19. Four year model simulation of waste volumes in the $2 \mathrm{H}$ evaporator system. 
The data also reports the available volume to store additional waste and salt cake in the evaporator system. The available volume is determined by subtracting the total volume of waste present in the system from the sum of the tank operating limits. The available salt volume is found by assuming that each receiving tank can hold $1000 \mathrm{kgal}$ of salt cake and sludge and subtracting the solids volume present in each tank from this capacity. Since some of the salt cake storage capacity may be occupied by liquid waste, the available volume for salt cake can exceed the total available volume. Figure 20 shows a plot of available capacities in the $2 \mathrm{H}$ evaporator system.



Figure 20. Four year model simulation of waste capacity in the $2 \mathrm{H}$ evaporator system.

When a receiving tank is in the process of salt removal, it is assumed to be unavailable for waste storage and is not included in calculating the available volumes until salt removal is completed. During salt removal, the total waste volume and salt volume may decrease while the available capacity also decreases as fresh waste is generated. When salt removal is completed in a receiving tank, there will be a sudden increase in available volume and salt storage capacity. This effect is observed in Fig. 20 for the $2 \mathrm{H}$ evaporator system.

The summary data also includes a report of the net space gain accomplished by the evaporator system during the previous time period. The net space gain during FY 1987 is the number reported at the start of FY 1988. Net space gain is defined as the total evaporator overhead production less any internal water additions. Therefore, the volume of 
desalt/descale water added to the concentrate tank and the steam dilution added to recycles are subtracted from the net overhead production to give the net space gain. Net overhead production is calculated as the difference between the integrated feed and concentrate volumes or $V_{o}($ net $)=I V_{f}-I V_{c}$ (see Appendix).

A one year listing of flow summary data from Option 5 is shown in Fig. 21. The volumes of total waste and salt cake entering and leaving each evaporator system are given. To balance inflow and outflow, all of the dissolved salt in waste solutions entering each system is converted into the equivalent amount of salt cake. This is different from the other summary reports that give the actual amount of salt cake made.

\begin{tabular}{|c|c|c|c|c|c|c|}
\hline Inflow/outflow Kgalo & $\begin{array}{c}\text { of waste } \\
1 \mathrm{~F} / 2 \mathrm{~F} \\
\text { volume }\end{array}$ & $\begin{array}{l}\text { volume and } \\
\text { Systein } \\
\text { Salt Cake } \\
\text { i }\end{array}$ & $\begin{array}{r}\text { salt as } \\
1 \mathrm{H} \\
\text { volume }\end{array}$ & $\begin{array}{l}\text { quivalent } \\
\text { system } \\
\text { Salt Cake }\end{array}$ & $\begin{array}{r}1 \text { t cake } \\
2 \mathrm{H} \\
\text { volume }\end{array}$ & $\begin{array}{l}\text { FY } 1987 \\
\text { System } \\
\text { Salt }\end{array}$ \\
\hline DWPF Recycle & .0 & .0 & .0 & .0 & .0 & $\overline{.0}$ \\
\hline Sludge wash water & .0 & .0 & .0 & .0 & .0 & .0 \\
\hline Waste from old tanks & .0 & .0 & .0 & .0 & .0 & .0 \\
\hline Transfer dilution & .0 & .0 & 40.5 & .0 & .0 & .0 \\
\hline Other additions & 1260.0 & 116.7 & 120.0 & .0 & 1650.0 & 126.8 \\
\hline Subtotal - & 1260.0 & 116.7 & 160.5 & .0 & 1650.0 & 126.8 \\
\hline Flxed canyon waste & 756.0 & 179.1 & 34.0 & 9.8 & 276.0 & 75.6 \\
\hline MTU/Tube waste & 494.9 & 117,3 & 587.1 & 174.4 & 94.5 & 25.9 \\
\hline Canyon subtotal - & 1250.9 & 296.4 & 621.1 & 184.2 & 370.5 & 101.5 \\
\hline External waste Input & 2510.9 & 413.2 & 781.5 & 184.2 & 2020.5 & 228.3 \\
\hline Inter-area transfers & .0 & .0 & .0 & .0 & .0 & .0 \\
\hline Lance steam & 435.3 & & 252.0 & & 272,6 & \\
\hline Recycle jet dilution & 415.9 & & 246.7 & & 149,1 & \\
\hline Process water & 837.2 & & 724.1 & & 254.4 & \\
\hline Total water added - & 1688.4 & & 1222.7 & & 676.2 & \\
\hline Total waste Inflow - & 4199.3 & & 2004.3 & & 2696.7 & \\
\hline Total overheads - & 4511.0 & & 2421.0 & & 3013.1 & \\
\hline Net space gain - & 2822.6 & & 1198.2 & & 2336.9 & \\
\hline Salt/Sludge removal. & .0 & .0 & .0 & .0 & .0 & .0 \\
\hline Inventory change -- & -311.7 & 413.2 & -416.7 & 184.2 & -316.4 & 228.3 \\
\hline
\end{tabular}

Figure 21. Material inflow-outflow summary for model predictions of total waste and salt cake volumes processed by the tank farm evaporator systems in fiscal year 1987.

The first two entries in the summary report are input streams to the $2 \mathrm{H}$ evaporator feed tank 43. DWPF recycle and sludge wash water are not operating during FY 1987. The old tank transfers are the volumes of waste removed in the retirement of type IV waste tanks sent to the low heat waste systems. In H-Area, this material is sent to tank 38 while in F-Area, the waste removal is sent directly to the $1 \mathrm{~F}-2 \mathrm{~F}$ evaporator feed tank 26 . The model simulation is programmed to add $4 \%$ water dilution to all transfer streams. This is appropriate for the H-Area transfers but should not be included in F-Area. The simplest way to account for this difference was to allow the $4 \%$ dilution in both streams and to correct the volume and specific gravity of the F-Area waste removal in the program to allow for the dilution. Therefore, the sum of the old tank transfers and the transfer dilution in F-Area equals the total volume of waste removed from the type IV tanks. It was expected that waste removal would only be used in FY 1986 simulations and it was not included in the present example. The model could be updated to remove these transfers from type IV waste tanks entirely. 
Transfer dilution in the $1 \mathrm{H}$ and $2 \mathrm{H}$ systems gives the total of all $4 \%$ water additions to inter-tank transfers, For the $1 \mathrm{H}$ system, these are transfers from the fresh waste recelpt tanks into tank 13 (previously into tank 30) and transfers into the evaporator feed tank. In the $2 \mathrm{H}$ system, transfers from tank 38 to tank 43 , sludge wash water additions, and the DWPF recycle stream are diluted.

Other additions are the miscellaneous waste volumes specified in the data base (Fig. A12). All of the waste sources listed above are summed in the first subtotal of the data summary to give the total external waste additions to the tank farm from sources other than canyon operations.

Waste additions from canyon operations are listed in the next two entries. The canyon waste is broken down into the fixed amount and the amount from material production as indicated in Eq. (56). These two components of the canyon waste are added to obtain the total contribution to tiunk furm waste from the canyon operations reported on the following line. The canyon waste is added to the subtotal of other external waste to obtain the total external waste input. For example, in FY 1987 for the 1F-2F evaporator system, 1260.0 $\mathrm{kgal}$ of waste from external sources plus another $1250.9 \mathrm{kgal}$ from canyon operations were added to the system for a total volume addition of $2510.9 \mathrm{kgal}$. This waste contained enough salt to form the equivalert of $413,2 \mathrm{kgal}$ of salt cake.

The data summary up to this point includes all of the waste added to the tank farm evaporator systems from external sources. This waste will contain all of the added salt. The lower part of the flow summary includes inter-area transfers, water additions from evaporator inefficiencies, and waste removal.

The inter-area transfers included in the data summary are those between the $2 \mathrm{H}$ and $1 \mathrm{~F}-2 \mathrm{~F}$ systems. When waste is transferred from the $2 \mathrm{H}$ system to the $1 \mathrm{~F}-2 \mathrm{~F}$ system, the amount appears as a negative number (indicating removal) on the $2 \mathrm{H}$ side and as the identical positive number on the 1F-2F side. The $4 \%$ dilution water is included in the $1 F-2 F$ transfer dilution amount. Since inter-area transfers do not occur until DWPF recycle is in operation, no transfers appear in the example data summary. Inter-area transfers are also required to move high heat waste from F-Area to the $1 \mathrm{H}$ evaporator system in H-Area. However, these transfers are considered to be internal to the $1 \mathrm{H}$ system and are not included in the summary.

The lance stearn, recycle jet dilution, and process water are internal water additions to the tank farm system from evaporator operations that decrease the overall efficiency in reducing waste volume. Lance steam is not included in the model calculations since it simple enters and leaves the evaporator independently of other process functions. However, the amount of steam lance that would be required is reported here for comparison to actual evaporator operating data. The recycle dilution is the water addition to transfers of concentrate from the receiving tanks to the feed tanks. Process water is the water added to the evaporator from desalt/descale flushing operations. These three volumes are summed on the line labeled total water added.

Total waste inflow is calculated as the sum of the external waste input and the total water added. This represents the volume of fresh waste added to the tank farm during the indicated time period. The total overheads is the net evaporator overhead production with the steam lance added back in. Net space gain from evaporator operations is equal to the total overheads less the total water added. Since the added water represents evaporator inefficiencies, the net space gain is the real gain in tarik farm capacity realized from operation of the evaporator system. Tank capacity is also recovered through waste removal 
operations and the next line in the summary reports the total amount of salt and sludge removed during this time perlod. Tho simulation only removes the amount of salt cake, or equivalent supernate, speciffed in the removal schedule. Some small volume of waste may therefore remain in the tank after salt removal is completed. Similarly, if less salt is present In the tank than was specified, the waste removal volume during the last step of a salt removal sequence may not be an integer value.

The final line of the inflow/outflow summary data reports the inventory change within each evaporator system. This value is calculated by adding up the total volume of waste and the equivalent salt cake present in all of the waste tanks within each system and taking the difference between the value at the start and end of the time period. If the net space gain exceeds the inflow of fresh waste, the volume inventory change is negative. If salt is removed from the system, the salt cake inventory change may be a negative number.

Enough information is presented in the inflow/outflow summary to perform overall material balances since the sum of the net space gain, the salt/sludge removal, and the inventory change must equal the external waste input plus inter-area transfers. For example, in FY 1987, for the 1F-2F system overall material balances on total waste volume and salt cake are:

\begin{tabular}{|r|c|c|}
\hline & Total Volume & Salt Cake \\
\hline $\begin{array}{r}\text { Net space gain: } \\
\text { Salt/sludge removal: }\end{array}$ & $2822.6 \mathrm{kgal}$ & \\
Inventory change: & $-311.7 \mathrm{kgal}$ & $0.0 \mathrm{kgal}$ \\
\hline External waste input: & $2510.9 \mathrm{kgal}$ & $413.2 \mathrm{kgal}$ \\
\hline
\end{tabular}

The overall material balances (including ones containing salt and sludge removal) have always checked to within 100 gal of waste material. In the low heat waste systems, the net volume change given by Option 5 is equal to volume changes reported in Option 4 . However, for the high heat waste system, the volume change in Option 5 is equal to the net waste addition to the entire system while the changes reported in Option 4 reflect internal transfers from the fresh waste receipt tanks to the evaporator system.

\section{CONCLUSIONS}

A computer model to simulate operation of the waste tank farm systems at the Savannah River Site has been developed. The model uses an analytical solution to the dynamical material balance equations around the evaporators and associated waste tanks to predict the volumes of liquid waste, salt, and sludge for the tanks in the evaporator systems. Simple models of in-tank precipitation and sludge processing have been included to provide a complete analysis for all waste tanks. Input parameters to the simulation include the MMP forecast of canyon operations in the separations areas, other waste sources, ovaporator operating parameters, and salt and sludge removal schedules.

The model determinos how the evaporators would operate, when waste transfers can be made, and waste accumulation rates. A stochastic element is included in the programming to allow random assignment of evaporator down-time during a simulation. In principal, 
this feature can be used to determine the probability that the tank farm will experience problems for a given set of operating conditions.

Output from the model includes predictions of individual waste tank contents, summaries of evaporator system operations, and reports of space gain and remaining capacity to store waste matertals within the tank furm. Model simulations can be made on a daily basis for up to 20 years. Calculations to simulate one year of tank farm operation require less than one minute of computer time on the Macintosh ${ }^{\oplus}$ II computer. Data entry and analysis consume the bulk of the real time required to assess tank farm operations. Model parameters have been validated by comparing simulations to known system operation during fiscal years 1985 and 1986. The model reproduces actual tank farm operntions with good accuracy. The computer model can be used to forecast waste tank farm utilization and capacity on an operational basis.

Most of the work developing and validating this model was performed during years 1986 through 1988. Publication of this report documenting the model was delayed for several years when the author was transferred to another assignment. Therefore, the example data shown in the report is somewhat dated but may still be used for illustration purposes to demonstrate the basic concepts of the model. Some of the assumptions used in the model development are also no longer valid as operation of the waste tank farm has changed over the past several years. Most of these changes are reflected in the differences between the flowsheets in Figs. $1 \mathrm{~b}$ and $1 \mathrm{c}$. The following list summarizes the changes needed in the existing version of the model to bring it into conformity with current operating practices.

1. Tank 32 is used as a receiving tank for fresh high heat waste in H-Area.

2. Tank 30 is a concentrate receiving tank for the $1 \mathrm{H}$ evaporator and no longer collects fresh high heat waste. All aged high heat waste currently goes to tank 13.

3. Tank 32 will replace tank 13 as the $1 \mathrm{H}$ evaporator feed tank in 1994 when the new $1 \mathrm{H}$ evaporator is installed.

4. If the new $1 \mathrm{H}$ evaporator needs to process DWPF recycle, it will be feed directly to tank 32 and not indirectly through tank 43 .

5. The provision for specifying separate canyon waste stream parameters for F-Area high heat waste prior to June 1986 can be deleted from the model and from the input.

6. If additional waste is removed from the H-Area type IV waste tanks for volume reduction, the material would be sent directly to tank 43 . In the future, wasto removed from old type I, II, and IV tanks may actually be sent directly to processing operations without first reducing the volume through ovaporation. In either case, the code logic to treat waste removal from old tanks must be revised and the input specification modified.

\section{REFERENCE}

1. "Savannah River Radioactive Liquid Waste Forecast - FY 1987," DPSP-87-1027, February, 1987. 


\section{APPENDIX}

\section{Summary of Model Equations}

When the recycle stream composition is specifled, the constants $K_{1}$ and $K_{2}$ are fully determined. The recycle specific gravity $\left(S_{r}\right)$ is evaporator dependent and, as explained above, $S_{\mathrm{r}}$ may also change between time steps if the recelving tank does not contain salt cake. Therefore, over a single time increment (one day), $\mathrm{K}_{1}$ and $\mathrm{K}_{2}$ remain constant.

$$
\begin{aligned}
& K_{1}=\frac{S_{0}\left(X_{0}-X_{r}\right)-R X_{r}}{S_{0} S_{s}\left(X_{s}-X_{r}\right)} \\
& K_{2}=\frac{S_{0}\left(X_{s}-X_{0}\right)+R X_{s}}{S_{0} S_{r}\left(X_{s}-X_{r}\right)}
\end{aligned}
$$

The volumetric flow quantities $Q_{1}, Q_{2}$ and $Q_{3}$ are also assumed to maintain constant values over the one day time increment. The selection of a one day time increment is based on the fact that tank farm operations usually occur on this time scale. Nothing in the model development lirnits the choice of an appropriate time step. However, smaller time increments would increase the computation time without significantly enhancing the simulation realism.

$$
\begin{gathered}
Q_{1}=V_{x} S_{x}+V_{r} S_{r}+V_{1} S_{1}+F V_{r} \\
Q_{2}=V_{x} S_{x} x_{x}+V_{r} S_{r} x_{r}+V_{1} S_{1} x_{i} \\
Q_{3}=V_{x}+V_{r}+V_{1}+F V_{r}
\end{gathered}
$$

The evaporator overhead rate $\left(V_{0}\right)$ will vary as a function of the feed specific gravity; however, to simplify the mathematical analysis, $V_{0}$ is treated as a constant over the time period of one day. That is, the evaporator overhead rate is calculated using the specific gravity of the feed tank at the start of the time step and is assumed to remain constant in solving the other material balance equations.

$$
V_{o}=K_{e}-(7560) S_{f}(0)
$$

When the evaporator is not operating, the overhead rate is set equal to zero. With $V_{0}$ determined, the remaining system constants are evaluated from the following equations:

$$
\begin{gathered}
F_{0}=V_{t}(0) S_{f}(0)\left[X_{0}-X_{f}(0)\right] \\
C_{1}=Q_{1} X_{c}-Q_{2}-V_{0} X_{c} \\
C_{2}=F_{0} \frac{\left[Q_{2}-Q_{1} X_{f}(0)\right]}{\left[X_{c}-X_{f}(0)\right]}
\end{gathered}
$$

The functions of the time increment may then be calculated. In the actual implementation, the time increment was fixed at one day. With the variables defined in a consistent set of 
units, (e.g. volumetric flow rates in gal/day), the time step $\tau$ would then be equal to one in all of the following equations:

$$
\begin{gathered}
G(\tau)=F_{0}+C_{1} \tau \\
H(\tau)=C_{2}\left[\frac{F_{0}}{G(\tau)}\right]^{p}, p=\frac{V_{0} X_{0}}{C_{1}} \\
J(\tau)=\frac{\left[H(\tau)+\left(Q_{2} V_{0} \tau\right)-C_{2}\right]}{\left[X_{0} Q_{1}-Q_{2}\right]}
\end{gathered}
$$

Finally, the mass fraction of salt in the evaporator feed, feed tark waste volume, and the volumes of salt cake and liquid waste in the receiving tank are calculated as

$$
\begin{gathered}
X_{f}(t)=\frac{\left[Q_{2} G(\tau)-X_{c} H(\tau)\right]}{\left[Q_{1} G(\tau)-H(\tau)\right]} \\
V_{t}(t)=\frac{\left[Q_{3} G(\tau)-\left(1-K_{a} X_{c}\right) H(\tau)\right]}{\left[X_{c} Q_{1}-Q_{2}\right]} \\
V_{s}(t)=V_{s}(0)+K_{1} J(\tau) \\
V_{1}(t)=V_{1}(0)+K_{2} J(\tau) \cdots V_{s} \tau
\end{gathered}
$$

Instantaneous values for the evaporator feed rate and concentrate production rate are determined from the relationships

and

$$
V_{f} S_{f}=V_{o}\left[\frac{X_{o}}{X_{o}-X_{f}}\right]
$$

$$
V_{c} S_{c}=V_{0}\left[\frac{x_{f}}{X_{0}-X_{f}}\right]
$$

It is of more interest to obtain values of $V_{f}$ and $V_{c}$ that are integrated over the time interval. This is accomplished by first integrating Eq. (10) to show that

$$
V_{s}(t)-V_{s}(0)=K_{1} s_{c} \int_{t_{0}}^{t} V_{c} d t
$$

Equations (A15) and (A19) are then used to show that

$$
\int_{t_{0}}^{t} V_{0} d t=I V_{0}=\frac{J(\tau)}{S_{0}}
$$


Using the relationship $V_{f}=V_{0}+V_{0}$ immediately gives

$$
\int_{t_{0}}^{t} V_{f} d t=I V_{f}=\left(V_{0} \tau\right)+\frac{J(\tau)}{S_{0}}
$$

The integral quantities, $I V_{f}$ and $I V_{o}$, give the total evaporator feed and concentrate volumes over the time increment $\tau$. These values are calculated and reported by the simulation program.

\section{List of Variables}

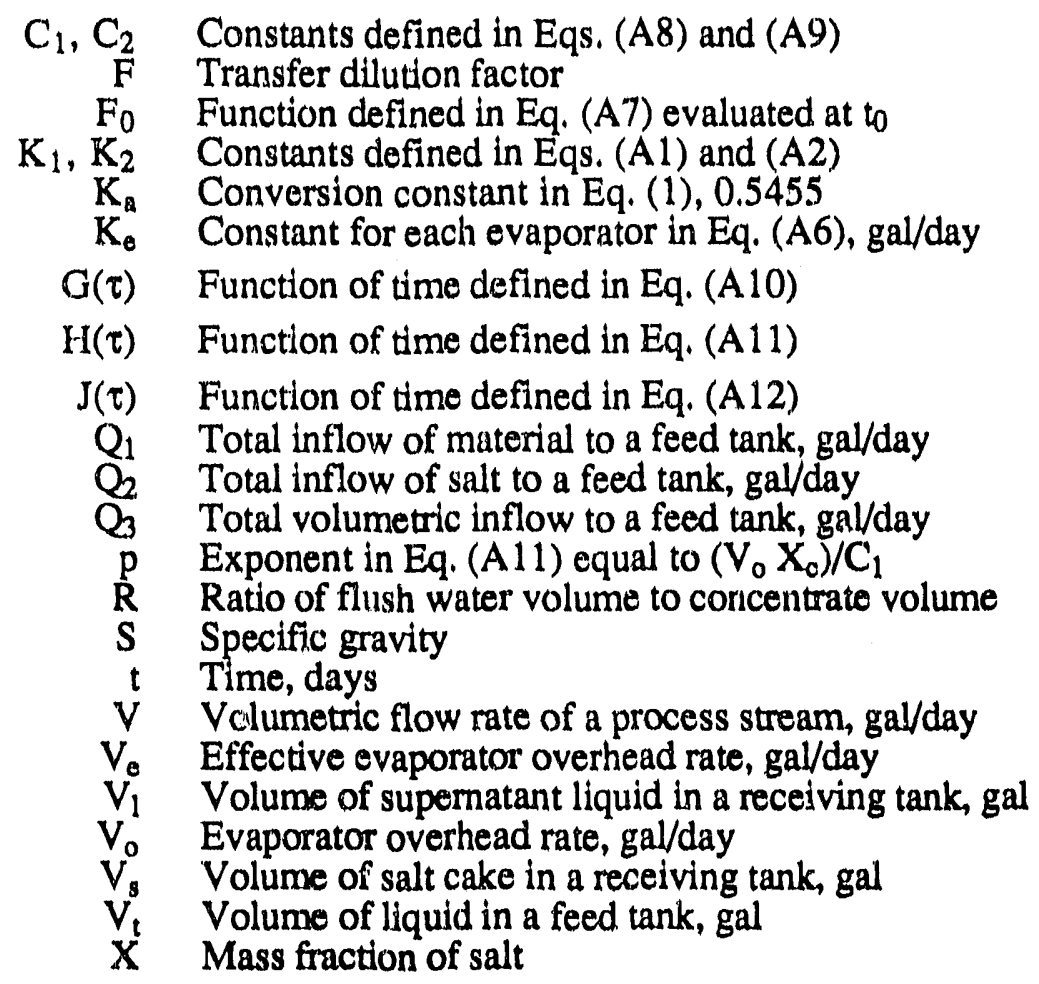

Subscripts:
c Evaporator concentrate stream
f Fieed tank and evaporator feed stream
i Waste from other sources
r Recycle stream
S Salt cake
$\mathrm{x}$ Waste from separation area canyon

Greek Symbols:
$\tau \quad$ Time increment, $\Delta \mathrm{t}=\mathrm{t}-\mathrm{t}_{0}$, days
$\rho$ Material density, lbm/ $\mathrm{ft}^{3}$ 


\section{User's Guide to Model Computer Programs}

This section provides a description of the three computer programs that comprise the waste tank farm model and the instructions needed to run them. The programs were initially written for an IBM ${ }^{\otimes} / \mathrm{PC}$ computer system and have since been converted to run on Apple ${ }^{\circledR}$ Macintosh ${ }^{\circledR}$ II computers as well. The following three codes and one data file must be present to run the tank farm simulation: WTFD, executable versions of WATFAM and READER, and TFARM.DAT. Specific instructions for each of the programs are provided below. Copies of the source code and executable programs may be obtai ad from the author. Examples of program input data and program output have already been presented in the discussion above.

\section{WTED}

The BASIC program WTFD can be used to create and edit the input data required for the model simulations. The version of the code that runs on a Macintosh ${ }^{\otimes}$ II computer has been modified to improve the programming and simplify its usage over what was available with the initial $\mathrm{IBM}^{\otimes}$ compatible version. The program can be executed by creating and running an application or by simply using the MICROSOFT QuickBASIC interpreter to load and run the program. The program can be run by starting the MICROSOFT QuickBASIC application and opening the WTFD file. This causes a display of the source code to appear in a screen window. Selecting START from the RUN menu in the BASIC interpreter will then begin program execution. Once the program starts, the BASIC menu is replaced with the WTFD program menu. More directly, the program can be run by simply selecting its file (i.e. double clicking on it) from the local directory which immediately executes the code and displays the WTFD program menu.

Once the progrwn is running, conirol is passed to a two entry pull-down menu within WTFD. The WTFD program menu has two headings: CONTROL and DATA SET. The user must first select the Read data file entry under CONTROL for the program to read the TFARM.DAT data set. This will be the only active menu selection when the program is first run. When the data set is successfully read, a message to that effect appears on the screen and the Read data file entry is no longer active in the menu. At this point, the other menu items become active.

The program then functions in two separate modes where simple key-stroke entries are used to move around within the data base and to edit the data:

1) Data Scanning Mode which allows the user to examine the input data by moving through pages of data as they are called onto the screen.

2) Data or Number Editing Mode which allows the user to edit numerical data entries.

The user chooses a particular set of data to examine by making a selection from the DATA SET menu. When a selection is made, the first page of the data will appear on the screen and a horizontal cursor bar is drawn by the first data entry. Menu selection is inactivated and the keyboard commands listed below become active:

Left Arrow $\leftarrow$ - Moves the cursor to the data entry to the immediate left of the current selection. If the extreme left entry is the current position, the cursor will wrap around to the extreme right entry. 


\begin{abstract}
Right Arrow $\Rightarrow$ - Moves the cursor to the data entry to the immediate right of the current selection. If the extreme right entry is the current position, the cursor will wrap around to the extreme left entry.

Up Arrow - Moves the cursor to the data entry immediately above the current selection. If the top entry is the current position, the cursor will wrap around to the bottom data entry.

Down Arrow - Moves the cursor to the data entry immediately below the current selection. If the bottom entry is the current position, the cursor will wrap around to the top data entry.

Page Up - Scrolls forward to the next page of data entries (this function is only active for the data sets too large to fit on a single page).

Page Down - Scrolls backward to the previous page of data entries (this function is only active for the data sets too large to fit on a single page).

END - This key must be pressed to clear the display of the current data page before another selection is made from the DATA SET menu.

HOME - Sends the cursor to the upper left hand data entry.

ENTER - Pressing this key once sets up data editing functions. Pressing this key a second time ends data editing.

HELP (ins) - Pressing this key once sets up data editing functions. Pressing this key a second time ends data editing.

$\mathrm{X}$ (del) - This key is designed to provide a simple cut and paste function. When the key is pressed, the number at the current cursor location is replaced by the contents of the key buffer. The buffer contents are reset whenever a number is edited and the final value entered. The buffer can be also set by positing the cursor at the desired number and pressing the ENTER key twice.
\end{abstract}

During data editing, the cursor changes shape to a vertical line positioned to the left of the active number and the following functions are available:

\footnotetext{
Left Arrow - Moves the cursor to the number to the immediate left of the current selection.
}

Right Arrow $\Rightarrow$ - Moves the cursor to the number to the immediate right of the current selection.

DELETE - Deletes the number to the left of the cursor location.

Entering numerical values in data editing mode inserts the number at the cursor location. The active editing keys are illustrated in Fig. Al, the data or number editing functions are written in italics. 


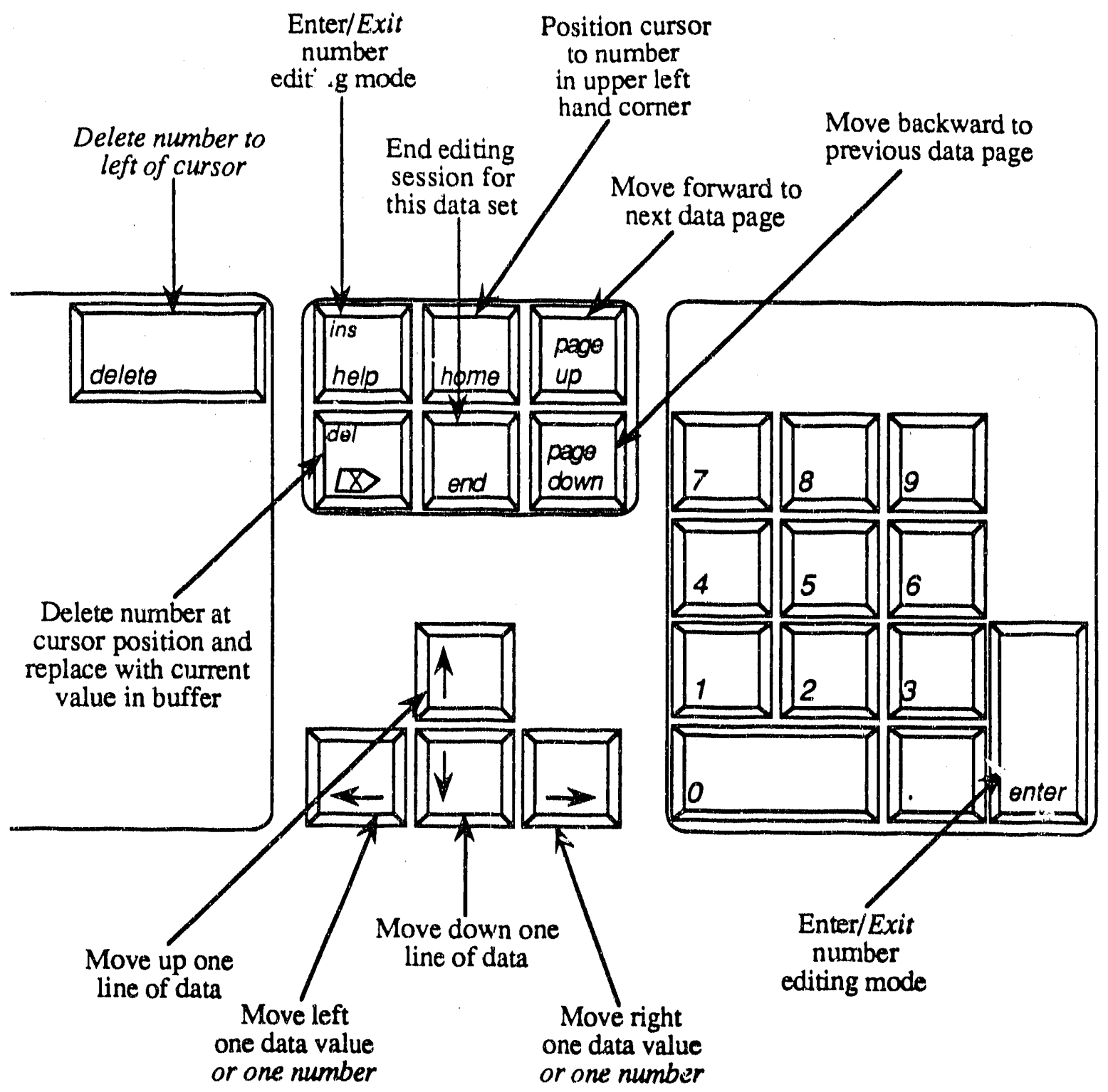

Figure A1. Diagram showing key functions active for WTFD editing.

To end WTFD the user must select the Stop - Write data entry under the CONTROL menu or the Quit - No changes entry. Here the user is given the option of saving the data changes or simply exiting the program. When the Stop. Write data option is chosen, the code creates a new data file named TFARM.NEW containing the altered data. This way the old data file (TFARM.DAT) is retained as a safety precaution and the user can rename the files as desired for future use. When the program is completed, the user is returned to the MICROSOFT BASIC interpreter and must close the file and quit the BASIC application program.

We note that other editors could be used to create and modify the data base. The codes that read the data have been set up to ignore lines beginning with an exclamation point (!). 
Lines containing the 1 symbol in the first column can then be inserted into the data base and used to include comments to clearly label the data. This feature is useful if other editors that can view the entire input file at once are used.

\section{WATEAM}

Once the data base has been created, running a model simulation is relatively simple. The simulation program is run by executing WATFAM and the only additional input required is:

1) Specifying the number of days to run the simulation. Note that the model assumes every year is 365 days long.

2) Specifying a printout interval by entering either the number of days between printouts or 0 for data printout at the start of each month.

3) Entering an odd random number between 1111 and 6777. This number is used to randomly assign days that the evaporators are not operating based on the input utility values. If the same random number is entered in different simulations having identical starting dates, the model will assign the same days for down-time. Varying the random number will give different simulations with the same input data.

4) Specifying whether full or partial log files are to be created. Log files are explained in more detail below.

When these input parameters are entered, the model simulation will start running. The program displays fiscal years on the terminal screen as they are being computed. Other output is written to the data files TANK.DAT and EVAP.DAT. In addition to these files, the program creates four log files (1F.LOG, 2F.LOG, 1H.LOG, and 2H.LOG) describing the operation of the evaporator systems. If full $\log$ files are specified, these files contain the following information:

1) Dates when recycles and transfers were wade in the evaporator system with identification of the tanks used.

2) Dates when the evaporator did not operate. These are the randomly assigned down times.

3) Dates when the evaporator stopped from lack of feed material and dates when it resumed operation.

4) Dates when shifts to the next receiving tank were made.

5) Dates when the evaporator stopped to wait for the salt removal from a receiving tank to be completed.

6) Error messages for tank overflows and the inability of an evaporator to handle the waste volume specified for type IV tank waste removal or sludge wash water in the fiscal year.

7) Messages indicating that salt removal from a receiving tank must be delayed since the tank is still in use when the scheduled removal begins. 
8) At the end of each fiscal year, the total number of days that the inter-area transfer line was used are reported if it is not zero.

For long term simulations, the log files can become quite large and difficult to read. Therefore, an option is provided to write condensed log files containing only the information in points 4) through 8). Examples of typical log files are given in the text of this report.

A structure chart for the WATFAM code is shown in Figs. A2a and A2b below. The branching from subroutine SOLVE has been split into three segments to more easily present the diagram. A brief description of the function of each subroutine in the WATFAM code is given in Tables Ala and Alb.
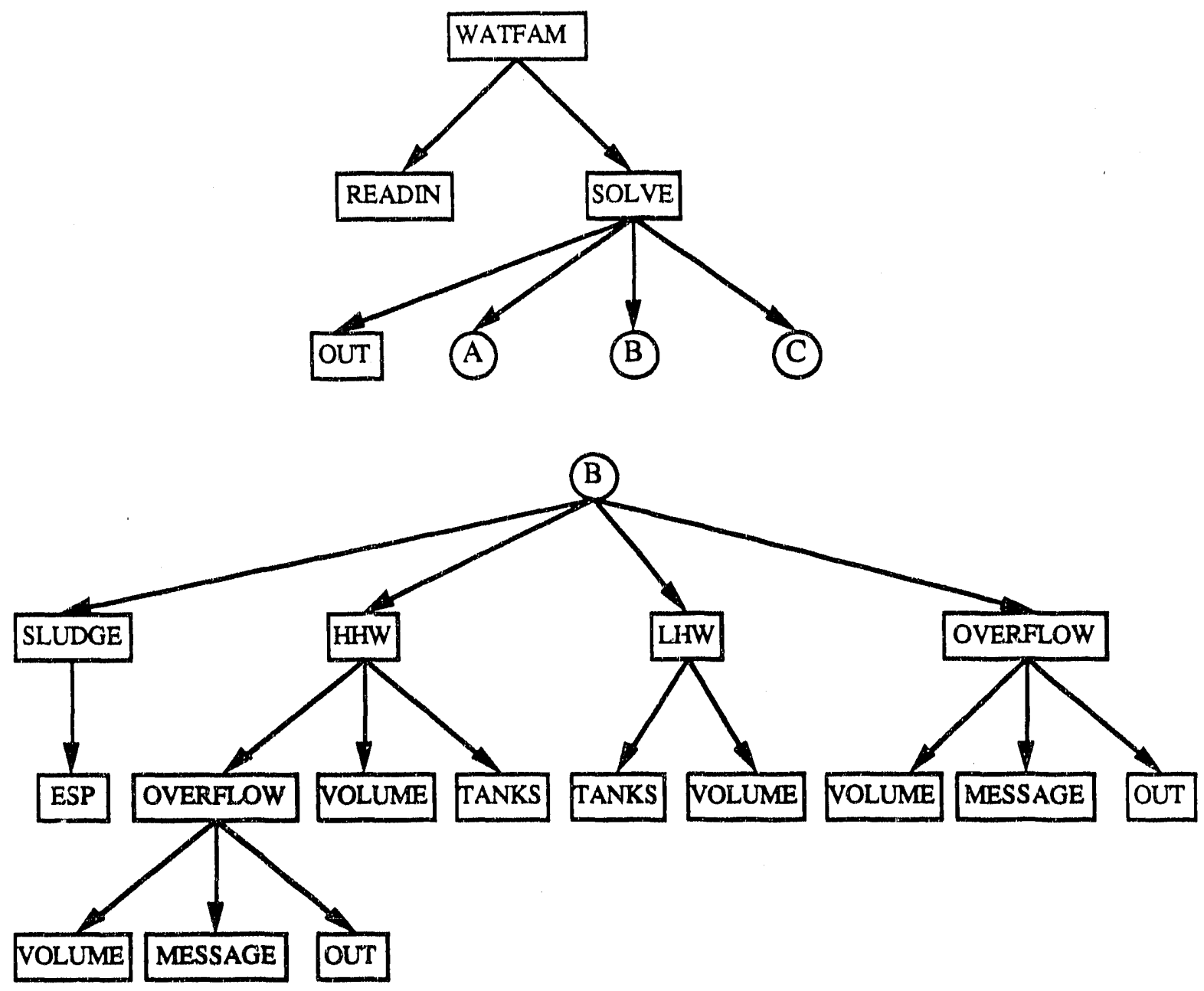

Figure A2a. Partial structure chart for WATFAM code. 

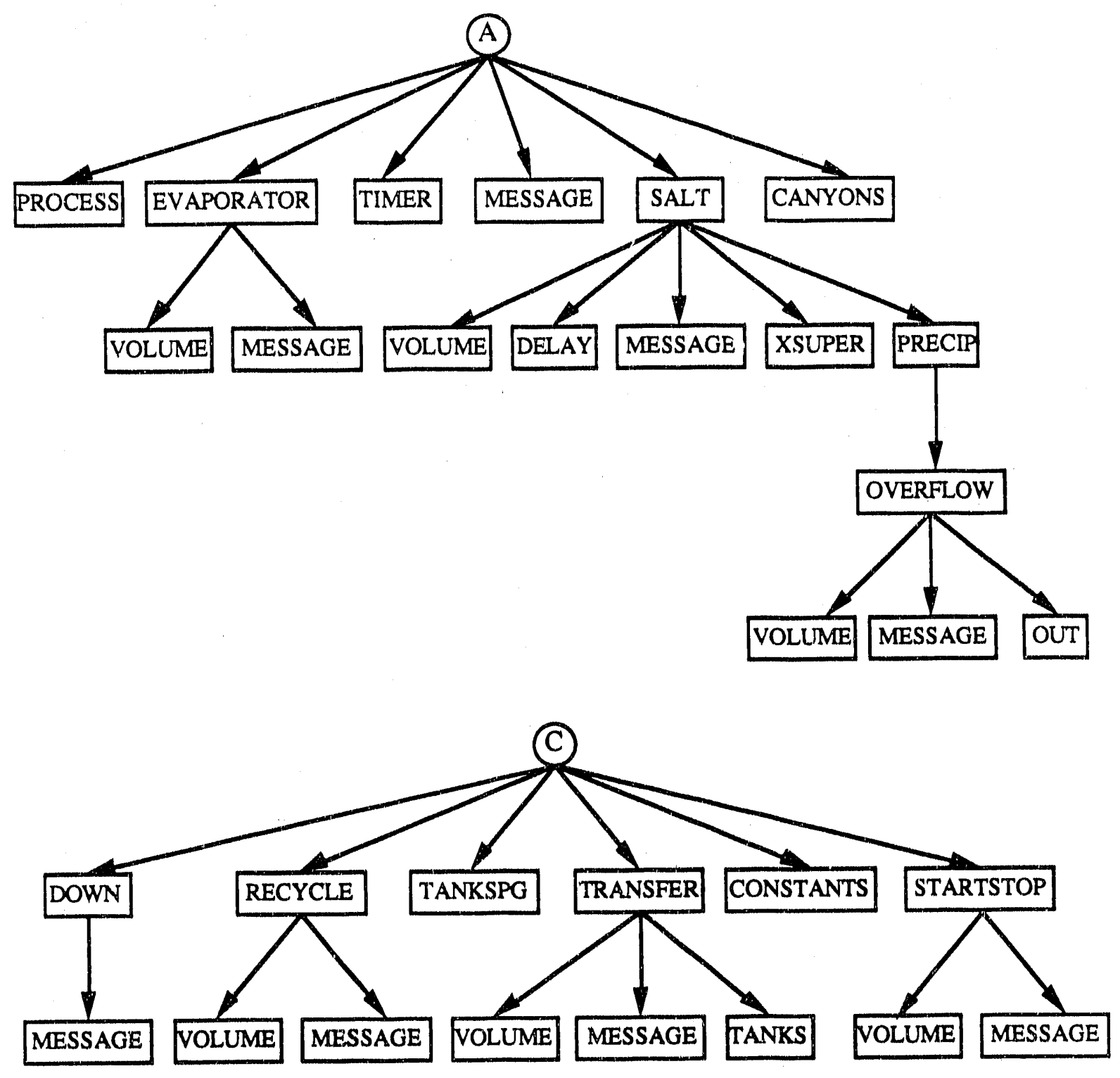

Figure A2b. Continuation of structure chart for WATFAM corle. 
Table A1a. Partial listing of subroutines in WATFAM code.

\begin{tabular}{|c|c|}
\hline Subroutine Name & Subroutine Function \\
\hline CANYONS & $\begin{array}{l}\text { Calculates volume and composition of high and low heat waste } \\
\text { streams from } \mathrm{F} \text { and } \mathrm{H} \text { Area canyons. }\end{array}$ \\
\hline CONSTANTS & Evaluates constant terms in material balance equations. \\
\hline DELAY & $\begin{array}{l}\text { Delays remaining salt cake removal schedule for an evaporator } \\
\text { system when removal begins in a tank that is being use as the } \\
\text { evaporator receiving tank. }\end{array}$ \\
\hline DOWN & $\begin{array}{l}\text { Randomly assigns days of the month when evaporator is not } \\
\text { operating. }\end{array}$ \\
\hline ESP & Models sludge processing operation in tanks 40,42 , and 51. \\
\hline EVAPORATOR & Sets operating parameters for the evaporator systems. \\
\hline HHW & $\begin{array}{l}\text { Adds fresh waste into the } \mathrm{F} \text { and } \mathrm{H} \text { Area high heat waste } \\
\text { systems. }\end{array}$ \\
\hline LHW & $\begin{array}{l}\text { Adds fresh waste into the } F \text { and } H \text { Area low heat waste } \\
\text { systems and performs inter-area line transfers. }\end{array}$ \\
\hline MESSAGE & Writes messages on evaporator system status to log files. \\
\hline OUT & Writes output to TANK.DAT and EVAP.DAT files. \\
\hline OVERFLOW & $\begin{array}{l}\text { Tests for tank over filling. If a tank has over filled, an error } \\
\text { message is written to the log file and the simulation is stopped. }\end{array}$ \\
\hline PRECIP & Models intank precipitation process in tanks 48,49 , and 50 . \\
\hline PROCESS & $\begin{array}{l}\text { Checks for start and stop of waste removal from old tanks, } \\
\text { DWPF, and sludge processing, and presence of new F canyon } \\
\text { evaporator, and new } 1 \mathrm{H} \text { evaporator. }\end{array}$ \\
\hline READIN & Strips out embedded comments from TFARM.DAT input file. \\
\hline RECYCLE & $\begin{array}{l}\text { Starts and stops the recycle of supernate from the concentrate } \\
\text { receiving tank to the evaporator feed tank. }\end{array}$ \\
\hline SALT & $\begin{array}{l}\text { Removes salt cake from receiving tanks based on input } \\
\text { schedule. }\end{array}$ \\
\hline SLUDGE & Removes sludge from tanks as specified by input schedule. \\
\hline
\end{tabular}


Table A 1b. Continuation of listing of subroutines in WATFAM code.

\begin{tabular}{|c|c|}
\hline Subroutine Name & Subroutine Function \\
\hline SOLVE & $\begin{array}{l}\text { Module that controls solution of material balance equations for } \\
\text { each evaporator systern. }\end{array}$ \\
\hline STARTSTOP & $\begin{array}{l}\text { Starts and stops the system evaporators depending on the } \\
\text { availability of feed material. }\end{array}$ \\
\hline TANKS & $\begin{array}{l}\text { Adjusts tank volume, specific gravity, and sludge content to } \\
\text { account for material inflow and outflow. }\end{array}$ \\
\hline TANKSPG & $\begin{array}{l}\text { Adjusts receiving tank specific gravity if it does not match the } \\
\text { specified value by forming or dissolving salt cake until the } \\
\text { desired specific gravity is reached. }\end{array}$ \\
\hline TIMER & Determines day, month, and year for current calculation step. \\
\hline TRANSFER & $\begin{array}{l}\text { Starts and stops transfers of waste material into the evaporator } \\
\text { feed tank. }\end{array}$ \\
\hline VOLUME & Determines tank volume and available space. \\
\hline WATFAM & $\begin{array}{l}\text { Driver module reads data from TFARM.DAT file and prompts } \\
\text { the user for other inputs. }\end{array}$ \\
\hline XSUPER & $\begin{array}{l}\text { Removes supernate liquid from tanks during salt removal and } \\
\text { from old waste tanks. }\end{array}$ \\
\hline
\end{tabular}




\section{BEADER}

Output from the model simulation is contained in the binary data files TANK.DAT and EVAP.DAT that are read using the READER computer program. A structure chart showing the relationship between the various subroutines in the code is shown in Fig. A3. A brief description of the function of each subroutine in the READER code is given in Table A2. The program is run by starting the READER application which displays a menu listing the following five reading options:

1) Data for an individual waste tank giving the volumes of total waste, salt cake, and sludge in $\mathrm{kgal}$ and in inches of tank level and the tank specific gravity on the reported date.

2) Data for all waste tanks of a particular type giving the volumes of total waste, salt cake, and sludge in $\mathrm{kgal}$ and in inches. Data can be obtained for type I, II, III, and IV waste tanks, high heat waste receiving tanks, and for all of the waste tanks in the tank farm.

3) Data for a particular evaporator giving the overhead, concentrate, and feed rates averaged over the output time period. The final specific gravity of feed material to the evaporator, the net space gain, and the percent of time that the evaporator was in operation are also reported.

4) A summary of evaporator system operations giving the total waste volume, salt cake, and sludge content for the $1 \mathrm{~F}-2 \mathrm{~F}, 1 \mathrm{H}$, and $2 \mathrm{H}$ evaporator systems. The available total volume, available salt volume, and net space gain are also given. The output is in $\mathrm{kgal}$ and can be read on a monthly or yearly basis.

5) A comprehensive summary of total inflow and outflow from each evaporator system listing all sources of waste volume and salt to the $1 \mathrm{~F}$ $2 \mathrm{~F}, 1 \mathrm{H}$, and $2 \mathrm{H}$ evaporator systems and waste disposal volumes on a monthly or yearly basis. The data can be used to show overall material balances around each system.

When a program option is selected, the user can choose to save the data in a disk file or display the data on the screen or both. When the data is saved, the user enters a name for the data file and a line of information to label the output. Use of the READER program and the various program options is largely self explanatory. Examples of the output are presented in the text of this report. 
WSRC-RD-91-17

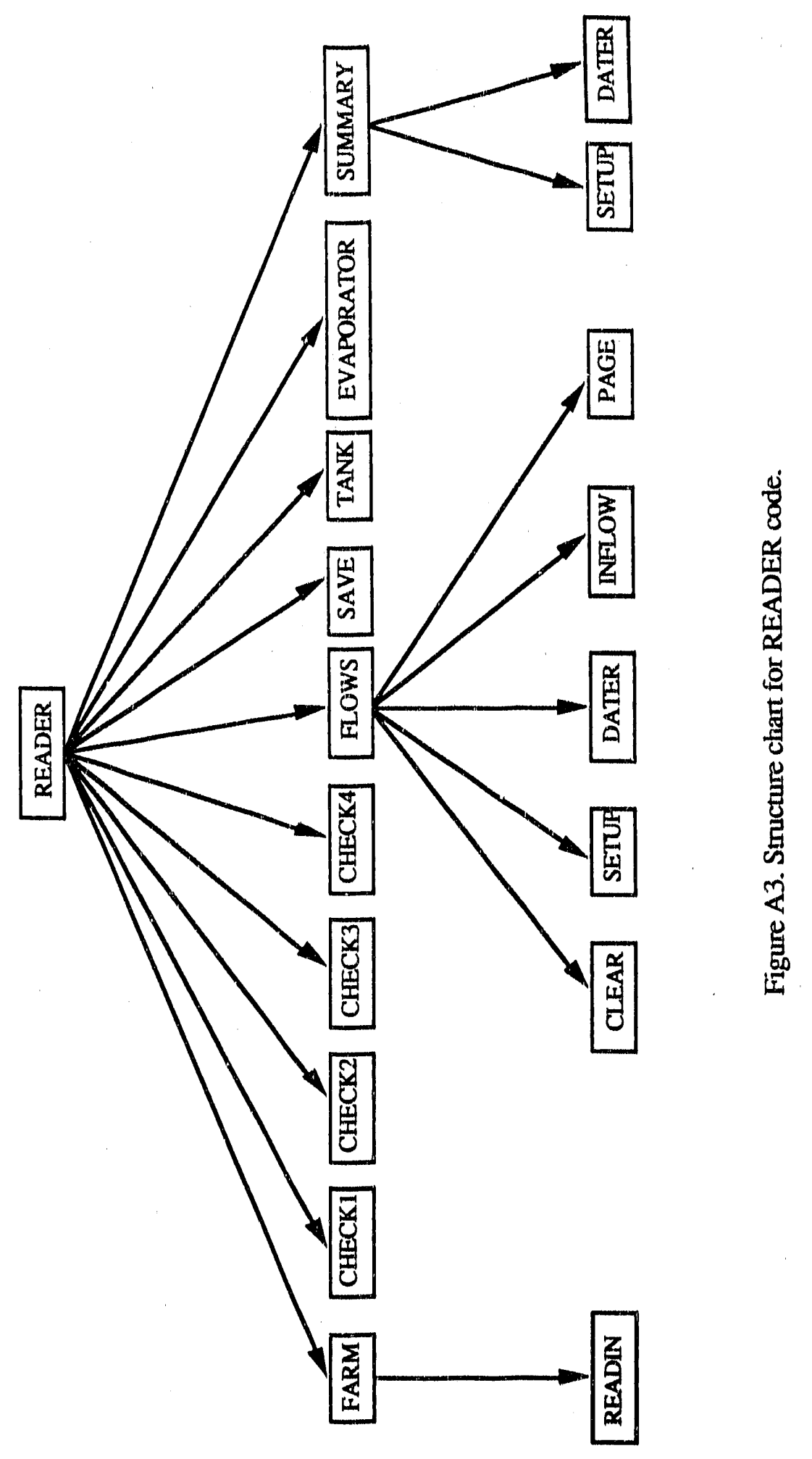


Table A2. Listing of subroutines in READER code.

\begin{tabular}{|c|c|}
\hline Subroutine Name & Subroutine Function \\
\hline CHECK 1 & $\begin{array}{l}\text { Prompts for input of tank number for data output with menu } \\
\text { option } 1 \text { and checks user response. }\end{array}$ \\
\hline CHECK2 & $\begin{array}{l}\text { Prompts for input of tank type for data output with menu } \\
\text { option } 2 \text { and checks user response. }\end{array}$ \\
\hline CHECK3 & $\begin{array}{l}\text { Prompts for input of evaporator system name for data output } \\
\text { with menu options } 3 \text { and } 4 \text { and checks user response. }\end{array}$ \\
\hline CHECK 4 & $\begin{array}{l}\text { Prompts for selection of yearly or monthly summaries of data } \\
\text { output with menu options } 4 \text { and } 5 \text { and checks user response. }\end{array}$ \\
\hline CLEAR & Sets array elements to zero. \\
\hline DATER & Creates formatted date for printing purposes. \\
\hline EVAPORATOR & $\begin{array}{l}\text { Reads simulation model output data for evaporator systems } \\
\text { from EVAP.DAT file. }\end{array}$ \\
\hline FARM & Reads model parameters from FARM.DAT file. \\
\hline FLOWS & $\begin{array}{l}\text { Creates data summary of material inflow and outflow to the } \\
\text { evaporator systems with option } 5 \text {. }\end{array}$ \\
\hline INFLOW & $\begin{array}{l}\text { Calculates volume and salt content of waste input from } \\
\text { canyons, DWPF, and miscellaneous waste sources. }\end{array}$ \\
\hline READER & Driver module that creates primary menu display. \\
\hline READIN & Strips out embedded comments from TFARM.DAT input file. \\
\hline PAGE & Writes a page of the inflow/outflow summary report. \\
\hline SAVE & Creates screen display flag or output files to report the data. \\
\hline SETUP & Sets parameters to check for printout dates. \\
\hline SUMMARY & Creates evaporator system data summaries. \\
\hline TANK & $\begin{array}{l}\text { Reads simulation model output data for individual waste tanks } \\
\text { from TANK.DAT file. }\end{array}$ \\
\hline
\end{tabular}




\section{Partial_Listing of Tank_Farm Data_Base}

A partial listing from an actual data base is presented in the following ten figures to 1llustrate the several types of input data required to run a model simulation. All of the figures are copies of screen displays generated by program WTFD.

\begin{tabular}{|c|c|c|}
\hline \multicolumn{3}{|c|}{ Process function daces: } \\
\hline SLmulation part: & 10 & 86 \\
\hline Waste removal on: & 10 & 85 \\
\hline Waste removal off: & 10 & 86 \\
\hline New E' can'yon evap: & 6 & 86 \\
\hline New 1 H evaporator: & 4 & 92 \\
\hline Sludge wash start: & 3 & 87 \\
\hline DWPF start: & 6 & 90 \\
\hline
\end{tabular}

Figure A4. Screen display of process dates.

Inttial conditions for the waste tanks: For October 1986

Waste tank number: 1

$$
\text { Fil1 } 11 \mathrm{mLt} \text { (1n): } 212
$$

Jet elevation (1n): 156

Total level (1n): 206.7

Sludge level (1n): 2,6

Salt level (1n): 201.1

Gallong per tnoh: 2710

specteto gravity: 1.372
2

221

193

214.8

1.5

193.7

2710

1.359
Pgup or PgDn for more data 3 1 5 264

24

17.4

17,3

2710

1.2

Figure A5. Screen display of first page of input data specifying waste tank initial conditions. 


\begin{tabular}{|c|c|c|c|c|c|}
\hline \multirow{2}{*}{\multicolumn{3}{|c|}{ Evaporator system parametors: }} & \multirow{2}{*}{$\begin{array}{c}\text { Before } \\
\text { L.H }\end{array}$} & Pgup or pgDn & \multirow{2}{*}{$\begin{array}{l}\text { for more data } \\
\text { Atter } 4 / 92 \\
\qquad 1 \mathrm{H}\end{array}$} \\
\hline & & $2 \mathrm{~F}$ & & $2 \mathrm{H}$ & \\
\hline Overhead (gal/day): & 8469 & 10871 & 10250 & 10660 & 25210 \\
\hline Lanoe (gath/day): & 1000 & 950 & 1000 & 950 & 1000 \\
\hline Overhead basis SPG! & 1.37 & 1.37 & 1.29 & 1.28 & 1.22 \\
\hline Flush water rat lo: & .14 & .065 & .11 & .07 & .08 \\
\hline Reoyole SPG: & 1.445 & 1.445 & 1.94 & 1,145 & 1.45 \\
\hline Feed tank number: & 26 & 26 & 32 & 43 & 32 \\
\hline
\end{tabular}

Figure A6. Screen display of evaporator system input parameters.

\begin{tabular}{|c|c|c|c|c|}
\hline \multicolumn{4}{|c|}{ Recelving tank schedule: } & or PgDn for more data \\
\hline Evaporatior: & $1 F$ & $2 F$ & $1 \mathrm{H}$ & $2 H$ \\
\hline $\operatorname{Tank} \# 1$ & 27 & 47 & 36 & 41 \\
\hline Tank \#? & 28 & 45 & 37 & 38 \\
\hline Tank \#3 & 25 & 44 & 30 & 41 \\
\hline Tank \#4 & 27 & 27 & 29 & 38 \\
\hline Tank \#5 & 28 & 28 & 31 & 41 \\
\hline Tank 6 & 75 & 25 & 36 & 38 \\
\hline Tank 7 & 0 & 47 & 37 & 0 \\
\hline Tank 8 & 0 & 44 & 30 & 0 \\
\hline Tank 9 & 0 & 45 & 29 & 0 \\
\hline Tank 10 & 0 & $9 \%$ & 0 & 0 \\
\hline
\end{tabular}

Figure A7. Screen display of input data to order the usage of the evaporator receiving tanks. 
Canyon wasto stream parameters:

(Unlt - Tube for H-Area, MTU for Fratea) Waste stream! F-LHW H-LiW

Before 6/86 AEter 6/86

Galions/Unte, 479

$35 \quad 121$

282

252

Los galt/Gal wagtel 3,2

3.7

4.1

3.9

3,9

GaL sludge/Undt, 26.2

1.33

$8 \cdot 4$

7.7

7.7

Figure A8. Screen display showing input parameters for canyon waste streams.

MMP forecast data and Evaporatior avaldablitity:

Pgup or PgDn for more data

\begin{tabular}{lllllll} 
& $10 / 86$ & $11 / 86$ & $12 / 86$ & $1 / 87$ & $2 / 87$ \\
\cline { 3 - 5 } H-Area Tubes: & 225 & 225 & 225 & 225 & 225
\end{tabular}

1 F Util1ty: .65

.65

.65

.65

.65

$2 \mathrm{~F}$ Utw11ty: .85

.85

.85

.8

.8

IH Ut111tys .75

.75

.7

.65

.65

24 Ut111tys $.7 \%$

.77

.77

.77

.77

MMP forecast data and

Evaporator avallabl11ty:

$\begin{array}{llllll}8 / 87 & 9 / 87 & \text { F'Y } 88 & \text { FY } 89 & \text { FY } 90\end{array}$

\begin{tabular}{|c|c|c|c|c|c|}
\hline H-Area Tubes? & 225 & 225 & 3608 & 4688 & 3463 \\
\hline F-Area & 02 & 82 & 1670 & 2075 & 1515 \\
\hline $1 F$ Ut111ty: & .55 & .55 & .5 & .6 & .6 \\
\hline $2 E U t 1.1 t y:$ & .85 & .85 & .7 & .83 & .83 \\
\hline 1H ULL11ty? & .7 & .7 & .7 & .7 & .75 \\
\hline $2 \mathrm{H} \mathrm{Ut} 111 \mathrm{ts}:$ & .8 & .8 & .81 & .81 & .81 \\
\hline
\end{tabular}

Figure A9. Screen displays showing the input data for the MMP forecast and evaporator fractional utilities on a monthly and yearly basis. 


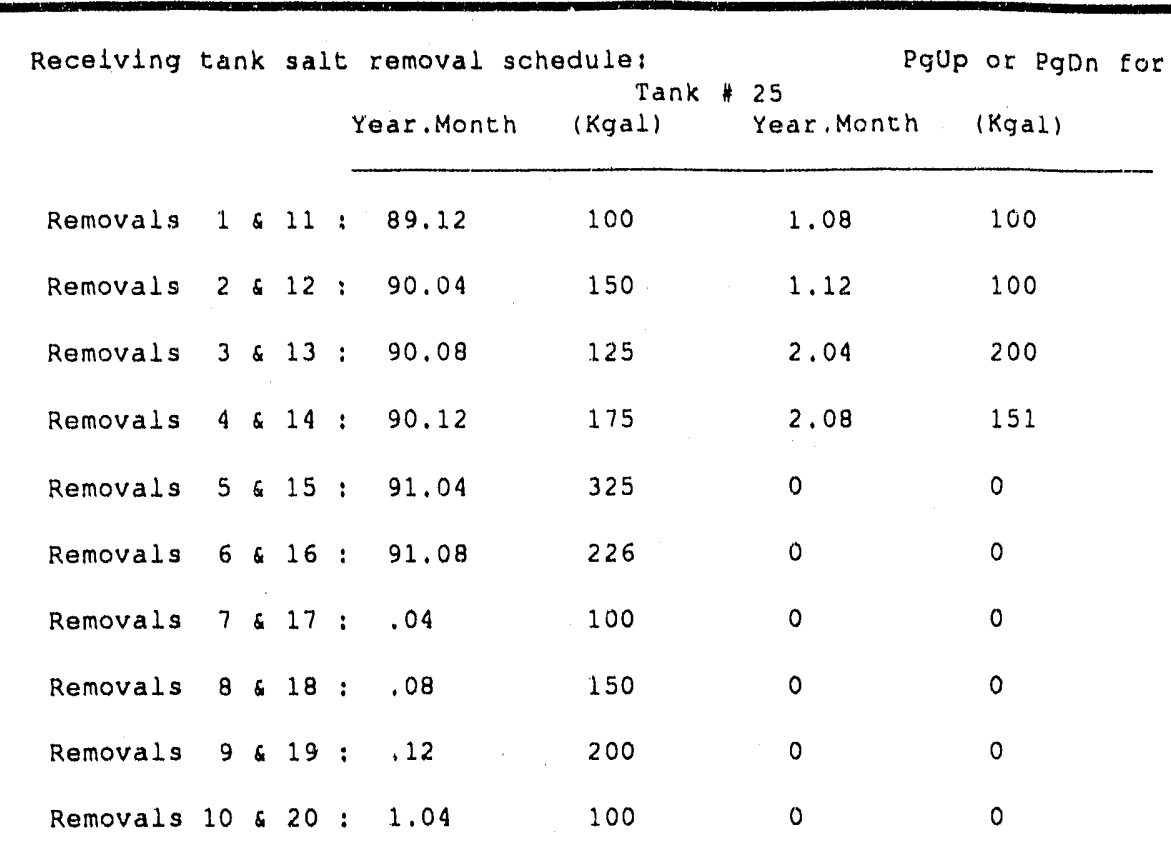

Figure A10. Screen display for input of salt removal schedule for tank 25.

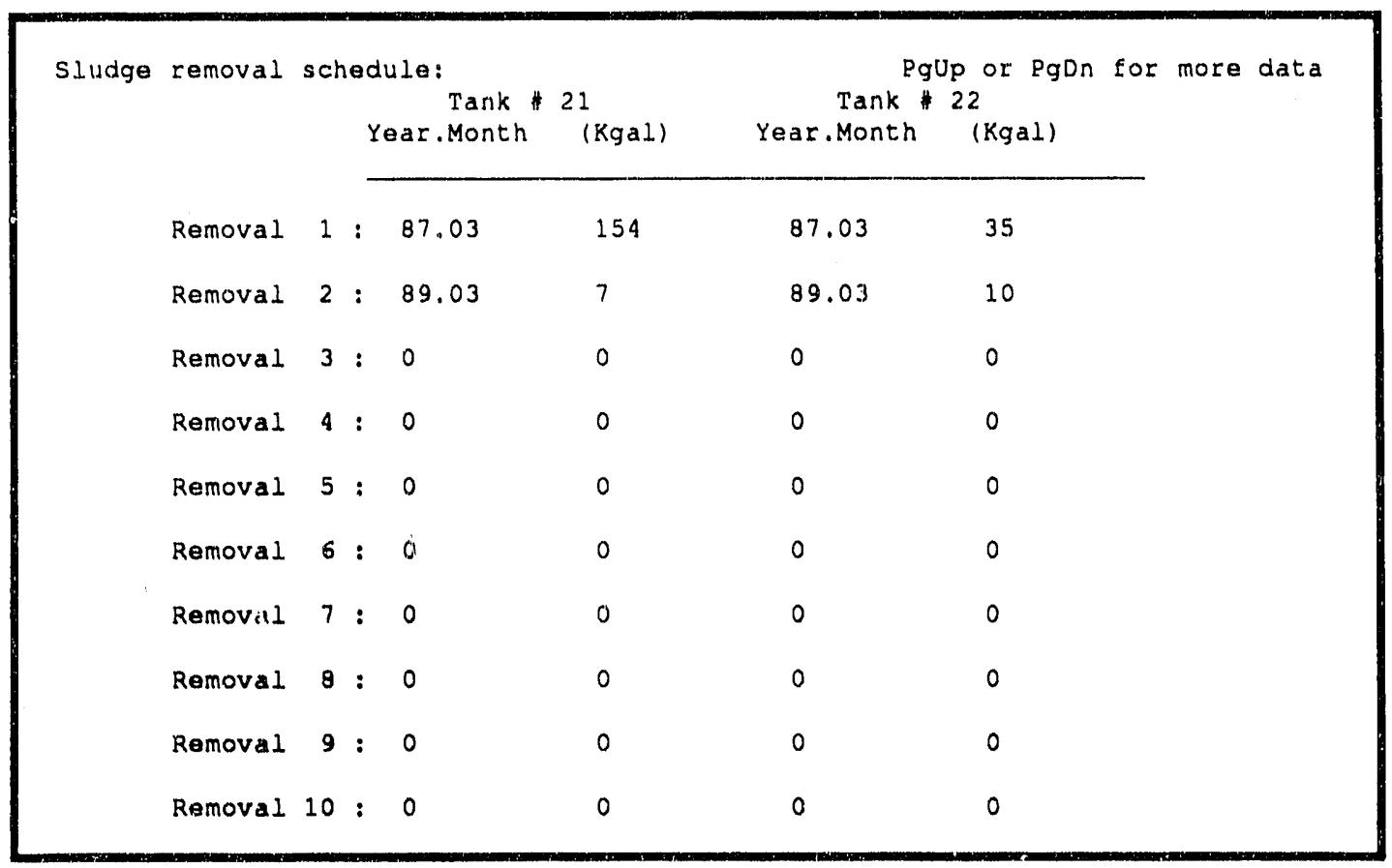

Figure A11. Screen display for input of sludge nemoval schedules for tanks 21 and 22. 


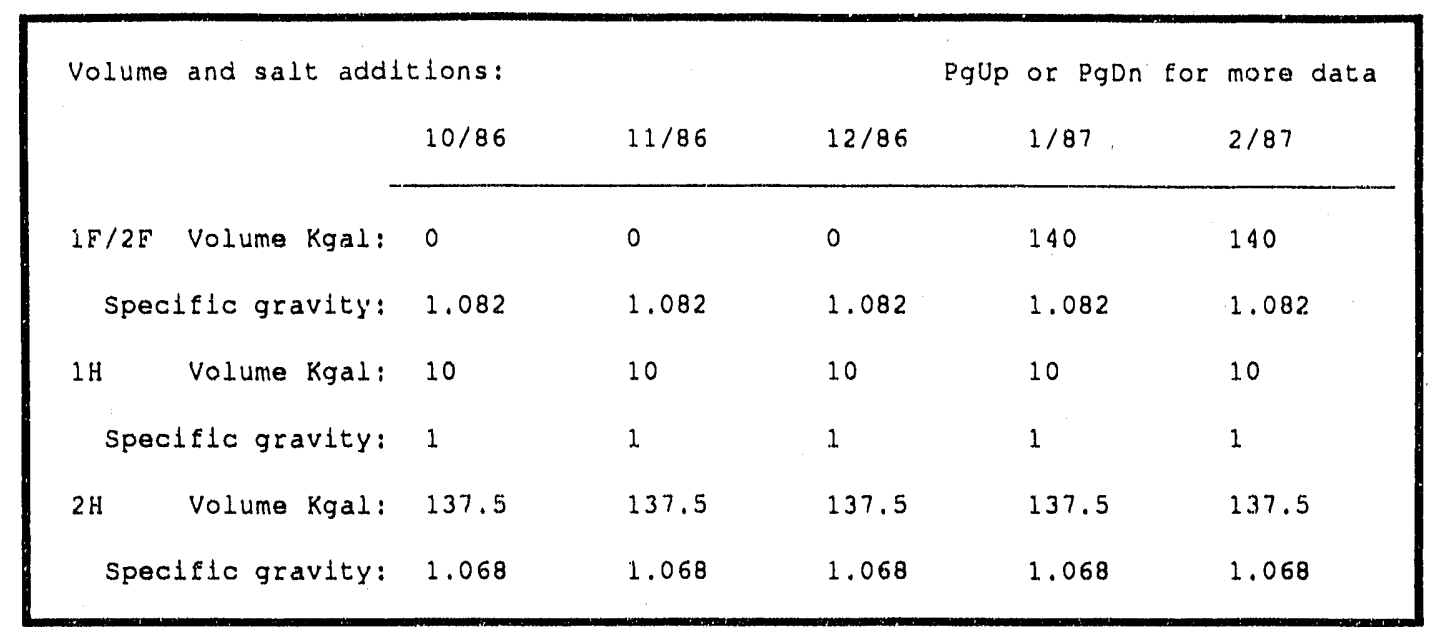

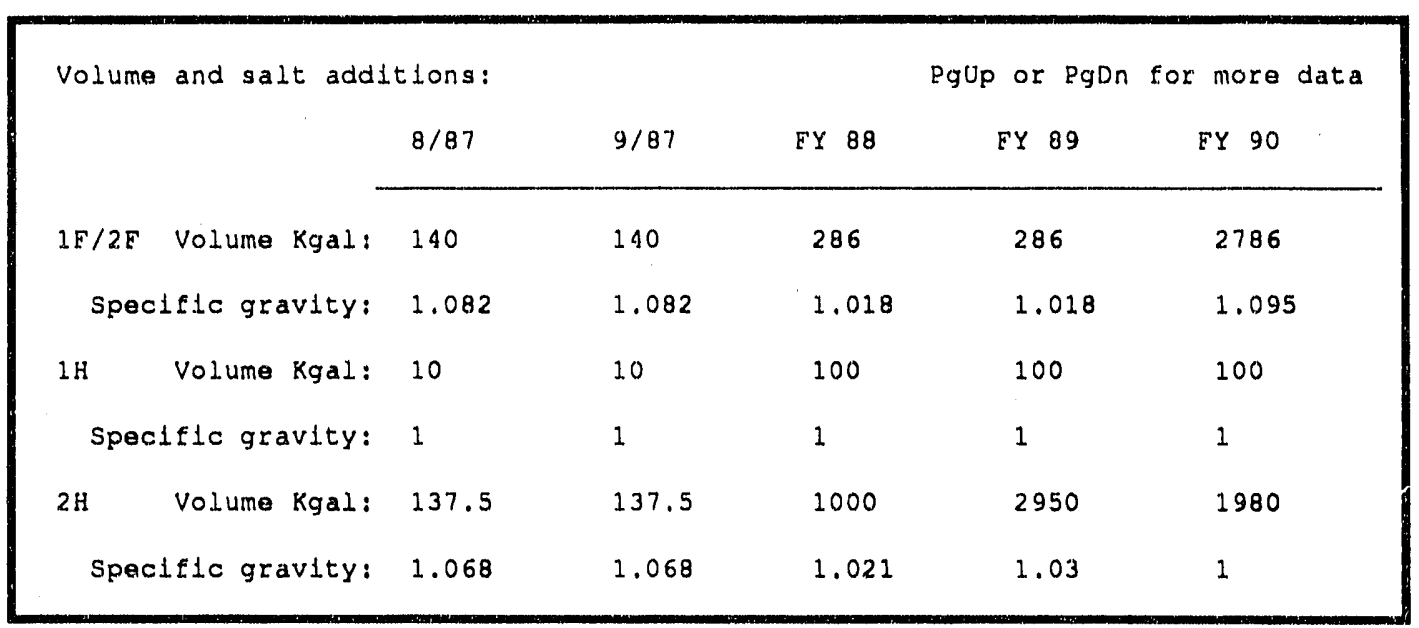

Figure A12. Screen displays showing input data for miscellaneous waste volume additions to each evaporator system on a monthly and yearly basis. 
Total waste removal from old tanks:

Pgup or PgDn for more data Between $10 / 85$ and $10 / 86$

F-Area Volume Kgal: 0

Specific gravity: 1.293

H-Area Volume Kgal: 0

Specifie gravity: 1.137

stream parameters:



Sludge

Pgup or PgDn for more data

Recycle

Wash Water

Kgal/Month: 0

0

Speciflc gravity: $1.017 \quad 1.026$

Sludge Kgal/Month: .64

0

Hlgh heat waste recelpt tank data:

Pgup or PgDn for more data

H-Area HHW tank: 39

Storage age (days): 5

F-Area HHW tank: 3 :

Storage age (days): 180

Figure A13. Screen display for miscellaneous input parameters. 


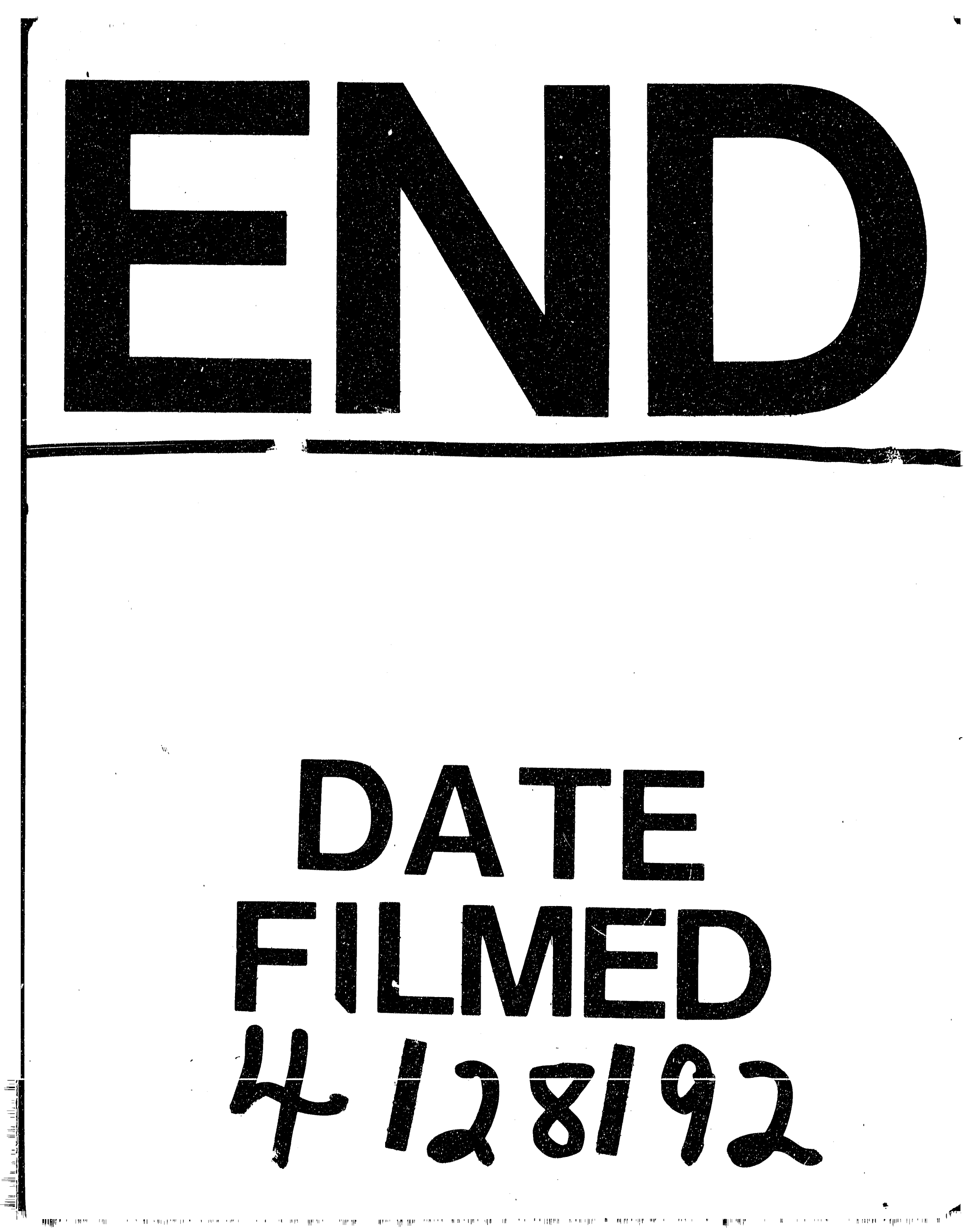


